$\operatorname{LnL}-14710$

2003002024

LBL -14718

\title{
Classical and Semiclassical Aspects \\ of Chemical Dynamics
}

Stephen Kevin Gray

(Ph.D. thesis)

'Lawrence Berkeley Laboratory

University of california

Berkeley, California 94720

August 1982

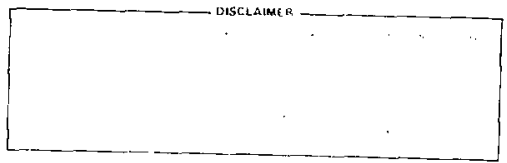

This work was supported in part by the Division of Chemical Sciences, office of Basic Energy Sciences, U.S. Department of Energy under contract ro. DE-ACOJ-76SF00098 and, in part, by a scholarship from the Natural Sciences and Engineering Research Council of Canada. Many of the computations were performed on a Harris $H 800$ minicomputer funded by National science Foundation grant CHE-79-20181. 
To my parents 
Classical and Semiclassical Aspects of Chemical Dynamics

by

\section{Stephen Kevin Gray}

\section{Abstract}

It is argued that a classical or semiclassical picture is useful for understanding a variety of problems in chemical dynamics. To demonstrate this, three very different problems are studied.

Tunnelir.g in the unimolecular reactions $\mathrm{H}_{2} \mathrm{C}_{2} \rightarrow \mathrm{HC}_{2} \mathrm{H}$, $\mathrm{HNC} \rightarrow \mathrm{HCN}$, and $\mathrm{H}_{2} \mathrm{CO} \rightarrow \mathrm{H}_{2}+\mathrm{CO}$ is studied with a classical Hamiltonian that allows the reaction coordinate and transverse vibrational modes to be considered directly. A combination of classical perturbation theory and the semiclassical WKB method allows tunneling probabilities to be obtained, and a statistical theory (RRKM) is used to construct rate constants for these reactions in the tunneling regime. In this fashion, it is round that tunneling may be important, particularly for low excitation energies. Nonadiabatic charge transfer in the reaction $\mathrm{Na}+\mathrm{I}^{+} \mathrm{Na}^{+}+\mathrm{I}^{-}$is treated with classical trajectories based on a classical Hamilconian that is the analogue of a quantum matrix representation. The charge transfer cross section obtained is found to agree reasonably well with the exact juantum results. An approximate semiclassical formula, valid at high energies, is also obtained.

The interaction of radiation and matzer is treated from a classical viewpoint. The excitation of an HF molecule in a strong laser is described with classical trajectories. Quantum mechanical results are also obtained and compared to the classical results. Althougn the detailed structure of che pulse time averaged energy absorpcion 
iv

cannot be reproduced classically, classical mechanics does predict the correct magnitude of energy absorption, as well as certain other qualitative features. The classical behavior of a non rotating diatomic molecule in a strong laser field is considered further, by generating a period advance map that allows the solution over many periods of oscillation of the laser to be obtained with relative ease. Classical states are found to form beautiful spirals in phase space as time progresses. A simple pendulum model is found to describe the major qualitative features.

With ain H. Mills 
Table of Contents

Page

Acknowledgements

viii

I. Introduction -- 1

II. Tunneling in Unimolecular keactions

A. Preliminaries -- 6

B. Simple Tunneling Correction to Unimoiecular

Rates, with Application to Vinylidene

Isomerization

7

C. Reaction Path Hamiltonian and the $\mathrm{HNC} \rightarrow \mathrm{HCN}$

Reaction

CI. The Reaction Path Hamiltonian

C2. An Improved Rate Constant Formula -

C3. Tunneling in $\mathrm{HNC}+\mathrm{HCN}-$

D. Tunneling in the Unimolecular Decomposition of

Formaldehyde

D1. Preliminaries -_- 27

D2. Reaction Path and Tunneling Calculations _....... 29

D3. A Closer Look at the Reaction Path 34

E. Sumnary - 38

III. Charge Transfer from a Classical Perspective

A. Preliminaries - 40

B. The Classical Analogue - 41

C. Classical Trajectories for $\mathrm{Na}+\mathrm{I}^{+} \mathrm{Na}^{+}+\mathrm{I}^{-}-\mathrm{N}^{----} 43$

D. A Semiclassical Model -..- 45 
E. Concluding Remarks - 52

IV. Classical Aspects of the Laser Excitation of Molecules

A. Overview

B. Classical and Quantum Mechanical Studies of HF in an Intense Laser Field

B1. Preliminaries - 54

B2. Methods - 55

B3. Results and Discussion 63

B4. Summary

C. The Period Adrance Map and the Simple Pendulum Model

C1. Preliminaries - 71

C2. The Period Advance Map - T3

C3. Application to a Dlatcmic in an Intense Laser

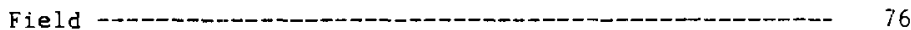

C4. The Pendulum Model

C5. Energy Absorption Trends -

C6. Concluding Remarks - 93

v. Concluding Remarks -

Appendix A. The Reaction Path 97

Appendix B. Initial Condittons for a Diatomic _... 99

Appendix C. Fixed Points of the Period Advance Map - 102

References - 
Page

Tables I-XIII - 1.2

Figure Captions 1-25 - 25

Figures $1-25-0-129$

Intended for Sir Isaac Newton in Westminster Abbey:

Nature and Nature's laws lay hid in night;

God said, Let Newton be! and all was light.

...b. Alexander Pope (1688-1744) 


\section{Accknowledgements}

I.t is a pleasure to acknowledge Professor Willian H. Miller for h1s great patlence, guidance, and support throughout these past four years.

I have also benefited from numerous and stimulating discussions with the following past and present members of our eclectic research group : Dr. Charles Cerjan, Dr. Boyd Waite, Dr. Lynn Hubbard, Shenghua Shi, Dominlc Al1, Steven Schwartz, Tucker Carrington, and especlally Peter Dardi, who deftly collaborated with me on some aspects of the laser-molecule problem.

The illuminating remarks of Professor Robert Wyatr, Dr. Yitzhak Weissman, and Professor M. A. Lieberman are also weeply appreciated.

The electronic structure wizardry of Dr. Yukio Yamaguchi, Dr. Yoshlihiro Osamura, and Professor H.F. Schaefei supplied the necessary fuel for the tunneling calculations described here. Dr. Híchel Dupuis is also to be chanked for providing me with a foolproof version of his electronic structure code.

Douglas Fox is to be thanked for maintaining our minicomputer and for providing much needed techntcal advice.

I would also liks to acknowledge some of my other good friends, who have kept me happy over the years : Saul Zackson, Shankar Subramanian, Bob Sherburne, Derek White, Doug Lichty, Doug Templeton, Jon Kowallis, John Verosky, and Bosco Weenie.

of course a special thanks is also due my fiancée, Anne Olson, whose patience, love, and understanding have been instrumental to the successful completion of this work. 
Finally, Professor James $S$. Wright, who initially sparked my interest in theoretical chemistry, is to be thanked for his continued encouragement, advice and frlendship.

My stay at Berkeley has been supported by a generous scholarship From the Natural Sclences and Englneering Research Council of Canada, as well as in part by the Division of Chemical sclences, Office of Basic Energy Sciences, U.S. Department of Energy, under contract no. DE-AC03-76SF00098. Many of the computations were performed on a harris H800 minicomputer funded by National Science Foundation grant CHE-79-20181. 


\section{Introduction}

Although molecules are inherently quantum mechanical in nature, there is great appeal, from both an aesthetic and practical viewpoint, to use classical and semiclassical methods in the description of basic molecular phenomena. The validfty of such approaches is limited, of course, to problems for which the de Bioglie wavelength may be considered small in comparison with, say, the dimensions of the interaction region.

The aesthetic appeal of classical mechanics, especially to chemists, is that molecular events, such as bimolecular collisions and reactions, may be depicted with classical crajectories - that is the coordinates and momenta of the relevent atoms may be followed explicitly as a function of time. This gives a mechanistic view of the reaction that has served frequently in the analysis and prediction of, for example, molecular beam experimenes ${ }^{1}$. The practicality of classical mechanics is that, given the potential of interaction, the solution of the classical equations of motion, i.e. Hamilton's equations, is not too difficult with the aid of modern computers, even for reactions involving as many as six or seven atoms ${ }^{2}$. In contrast, reactive quantum scatcering calculations are complicated by the man: channels, or basis functions, that must be included, as well as by serious convergence problems. It is amusing to consider a famous test case of chemical dynamics, the $\mathrm{H}+\mathrm{H}_{2}$ 
hydrogen atom exchange reaction in $3 d$. The first numericaliy accurace classical trajectory prediction of the various cross sections for this reaction, as a function of collision energy, was made in 1965 by Karplus, Porter, and Sharma ${ }^{3}$. Only in 1975, and with the expense of almost excessive human and computer $t$ ime, was an accurate quantum solution obtained ${ }^{4}$, and this solution was for a vary small range of collision energies. At present (1982) the solution for $\mathbf{n}+\mathrm{H}_{2}$ is still the only example of an accurate quantum solution for a realistic (i.e. no constraints such as collinearity) reactive chemical system. In contrast, many classical trajectory studies have appeared, for reactions ranging from ${ }^{5} \mathrm{~F}+\mathrm{H}_{2}+\mathrm{HF}+\mathrm{H}$, to ${ }^{6} \mathrm{H}+\mathrm{C}_{2} \mathrm{H}_{4}+\mathrm{C}_{2} \mathrm{H}_{5}$. Of course reactive quantum scattering theory is far from dead, and several groups are working on problems associated with it. Benchmark calculations for other three atom systems, such as $F+H_{L}$, are expected in the near future. For a discussion of the current status of reactive quantum scatcering theory, see Wyat t's recent review' ${ }^{7}$. However it would be fair to say that a full quantum solution of, say, $\mathrm{H}+\mathrm{C}_{2} \mathrm{H}_{4}$, will not appear in the near future and may never appear, owing to the immense practical difficulties.

of course quantum effects, ultimately arising from the uncertain:y principle and superposition, are observable in many experiments and approximate quantum theories may be used to describe them. A partic ularly appealing approximate quantum theory is aemiclassical mechanics ${ }^{8}$. 
Semlclassical mechanics constructs essentially a limiting or asymptotic solution to the Schrodinger equation for the case $h+0$, which is the classical limt of small de Brogile waveiength. The well-known ${ }^{9}$ WKB tunneling and quantization formulae are examples of semiclassical analysis. The result of taking the classical limit is that classical trafectories, and even a more generalized trajectory that may be complex-valued, are retrieved. However, these trajectories are used in constructing complex amplitudes that must be added before an overall probability, the square of the $S$ matrix, may be obtained 8 . Most chemically interesting quantum effects, including interference oscillations in cross sections and tunneling, can arise out of a semiclassical treatment. To obtain an "exact" asymptotic solution of the Schrodinger equation for anything but a simple system has turned out to be almost comparable in difficulty to actually solving the Schrodinger equation directiy. But the concepts involved in semiclassical mechanics have greatly alded in the interpretation of many results in chemfcal dynamics and, moreover, approximate semiclassical methods, such as the WKB method, are exceedingly easy to apply and often can be quite accurate.

The present thesis applies these classical and semiclassical approaches to a vartety of chemical dynamics problems. In sec. II, tunneling in the unimolectilar reactions $\mathrm{HNC}+\mathrm{HCN}, \mathrm{H}_{2} \mathrm{C}_{2} \rightarrow \mathrm{HC}_{2} \mathrm{H}$, and $\mathrm{H}_{2} \mathrm{CO}+\mathrm{H}_{2}+\mathrm{CO}$, is treated with a classical Hamiltonian 
that allows the "reaction coordinate" and transverse vibrational modes to be considered directly ${ }^{10}$. A comblnation of classical pertc"uation theory and the WKB method allows tunneling probabilities to be obtained, and a statistical theory (RRKM) is used to construct rate constants for these reactions due te tunneling ${ }^{11}$. In this fashion it is found that tunneling may play a significant role in the microcanonical rate constant, particularly at low excitation energies.

In sec. III, nonadiabatic reactions, such as the charge transfer reaction, $\mathrm{Na}+\mathrm{I} \rightarrow \mathrm{Na}^{+}+\mathrm{I}^{-}$, are considered. These sorts of reactions involve different quantum mechanical electronic states and thus are generally thought of as being pure quantum events. However, a novel classical hamiltonian treatment, due to Miller, Meyer, and McCurdy ${ }^{12}$, allows one to take the quantum mechanical matrix elements and construct a classical Hamiltonian. It is shown that purely classical crajectories can then reasonably approximate the charge transfer cross section for $\mathrm{Na}+\mathrm{I}$. An approximate semiclassical formula, based on this classical Hamilconian, and valio at high energies, is also derived.

In sec. IV, the interaction of radiation and matcer is treaced classically. The excication of an HF molecule by a strong laser is described with classical trajeccories. Quantum mechanical results are also obtained and compared to the classical results. As might be expected, averaged quancities are well described by che classical analysis. However, the decailed structure, including rotations, of che classical and quancum energy absorption spectra do have significant 
differences. The classical behavior of a nonrotating diatomic molecule in a strong laser is also considered, borrowing some methods from nonlinear dynamics. A method of obtaining the solution over many periods of oscillation of the laser, by generating a period advance map ${ }^{13}$, is given. The classical solution is seen to consist of spirals in phase space, representing very graphically the nature of the resonance between molecule and laser. Through certain approximate cransformations, it is possible to represent the problem as a simple pendulum ${ }^{14}$, with the momentum of the pendulum being related to the energy of the molecule, and the angle of the pendulum being the difference between molecular and laser phases. Finally, sec. $V$ gives some brief concluding remarks. 


\section{Tunneling in Unimolecular Reactions}

\section{A. Preliminaries}

Unimolecular reactions, such as molecular decompositions and rearrangements, have been extensively studied, and textbooks such as that of Robinson and Holbrook ${ }^{15}$, and review articles such as that by Hase ${ }^{16}$, give detailed accounts of many aspects. One aspect rarely discussed, however, is the role of quantum mechanical tunneling. One reason is that the effect of tunneling on the thermal rate constant is expected to be quite small. However, tunneling may play an important role in the microcanonical (energy-dependent) rate constant ${ }^{11,17}$, particularly at low excitation energies of the molecule. Today's laser techniques allow, in fact, an experimental determination of state specific rate constants, and some interesting examples include the work of Reddy and Berry ${ }^{18}$ on the methyl and allyl isocyanide isomerizations. Indeed, if a laser couid excice a molecule to an energy below its classical threshold for reaction, the entire rate of reaction measured would be due to tunneling. This feat, however, has not yet been accomplished in the laboratory.

In sec. IIB, we outline the simple tunneling correction to unimolecular rates given by Miller ${ }^{11}$ (see also Forst ${ }^{17}$ ), and apply it to the isomerization of vinylidene to acetylene. An explanation of the experimental difficulty in observing vinylidene is made on the basis of these resulis. Next, in sec. IIC, the reaction pach 
hamilconian of Miller, Handy and Adams ${ }^{10}$, for polyatomic systems, is discussed. A WKB runneling probability formula is developed with this Hamiltonian, and then applied to tunneling in the hydrogen isocyanide isomerization, $\mathrm{HNC}+\mathrm{HCN}$. In sec. IID the unimolecular decomposition of formaldehyde, $\mathrm{H}_{2} \mathrm{CO}+\mathrm{H}_{2}+\mathrm{CO}$, is discussed in rerms of the present semiclassical reaction path model.

B. Simple Tunneling Correction to Unimolecular Rates, with Application to Vinylidene Isomerization

RRKM theory (so named after its originators O.K. Rice, J. Ramsperger, L.S. Kassel and R.A. Marcus) is the basic theory of unimolecular reactions ${ }^{15,16}$. It may be considered a transition state theory because it assumes the existence of a transition state or critical configuration which may be considered the point of no return. If a molecule atcains this configuration during the course of reaction, then it is assumed to react, and not return to reactants. This concept will be modified below to allow a certain leakage due to tunneling. RRKM theory is a statistical theory because it assumes that all possible quantum states of the transition state (ano also reactants) are equally probable. As a corollary to the above two. assumptions, energy re-distribution within the molecule is considered to be faster than unimolecular reaction. Within these approximations, the microcanontcal rate constant is ${ }^{11}$

$$
k(E)=N^{\ddagger}(E) /[2 \pi h \rho(E)] \quad, \quad I I-1
$$


where $N^{*}(E)$ is the sum of states at the transition state, and $\rho(E)=d N_{0} / d E$ is the density of states for the reactant. More explicicly, the sum of staces for the cransition state and reactant are given by

$$
\begin{aligned}
& N^{\ddagger}(E)=\varepsilon_{\underline{n}} h\left(E-E_{\underline{\sim}}^{\ddagger}\right) \\
& N_{0}(E)=\varepsilon_{\underline{\sim}} h\left(E-\varepsilon_{\underline{\sim}}\right)
\end{aligned}
$$

where $h(x)$ is the usual step function,

$$
h(x)=\left\{\begin{array}{l}
0, x<0 \\
1, x>0
\end{array},\right.
$$

and $\varepsilon_{n}^{\ddagger}$ and $\varepsilon_{\underline{n}}$ are vibrational energy levels of the rransition state and reactant. The sums in eq. II-2 are over all possible quancum number combinations $\mathrm{n}=\left(\mathrm{n}_{1}, \mathrm{n}_{2}, \ldots\right)$. If is common co use a separable hamonic oscillator (or normal mode) approximation to obtain the energy levels, with $\left\{\omega_{i}^{*}\right\}$ and $\left\{\omega_{i}\right\}$ being the respertive hamonic frequencies. Thus, measuring the energy relative to the reactant minimum of energy,

$$
\begin{aligned}
& \varepsilon_{\underline{n}}=\sum_{i=1}^{F} h \omega_{i}\left(n_{i}+1 / 2\right) \\
& \varepsilon_{\underline{n}}^{*}=v_{0}^{\neq}+\sum_{i=1}^{F-l_{h} \omega_{i}^{*}}\left(n_{i}+1 / 2\right),
\end{aligned}
$$

where $F$ is the number of degraes of freedom and $v_{n}^{*}$ is the barrier 
height relative to the reactant (wichouc any zero-point energy corrections). Notice that the transition state is sumed over only $F \quad$; degrees of freedom, since the reaction coordinate $s$ is assumed not to be harmonic, but racher to have a potential energy profile $v_{0}(s)$, with a batrier at $s=0 \cdot\left(v_{0}(0)=v_{0}^{*}\right)$.

The key fdea is to replace the sum of statrs at the transition state, eq. II-2a, by a sum of tunneling probabilities if the total energy $E$ is below the classical threshold, $E_{\text {th }}=v_{0}^{\ddagger}+\varepsilon_{i=1}^{F-I} h_{i}^{\ddagger} / 2$ :

$$
N^{\ddagger}(E)+T(E)=\varepsilon_{\underline{n}} P\left(E-E_{n}^{\ddagger}\right)
$$

where $P\left(E_{S}\right)$ is the id cunneling probabilicy for energy $E_{S}$ in the reaction coordinate. If $v_{0}(s)$ is only known neat $s=0$, the cransition scace, one could use, for example, an Eckart potential barriet assumption and the corresponding cunneling probabilicy ${ }^{11,18}$. Alcernatively, if $V_{0}(s)$ is reasonably well defined, one could use the wKB method ${ }^{9}$ to obtain P. With eq. II-3, the microcanonical rate constant in the cunneling energy regine is given b, 11

$$
k(E)=T(E) /[2 \pi h \rho(E)] \quad \text { II-4 }
$$

As a final point, angular momentum has beer neglected in the above considerations. Miller ${ }^{11}$ has show that ics inclusion is not difficult, and experience has shown 2 ts effec: is not great fo: 
typical low total angular momentum situations.

To use the theory in its simplest form; one needs to know the geonetries and total electronic energies of the reactant, transition state, and preferably also the product. One also needs to know the normal mode frequencies of the reactant and transition state. To obtain these, it is necessary to evaluate second derivatives of the Born - Oppenheimer electronic energy with respect to the nuclear coordinates. The normal mode analysis then consists of diagonalizing the appropriately mass - weighted second derivative or force consiant matrix (see sec. IIC). For the reactant, one obtains $F=3 N-6$ positive efgenvalues corresponding to twe $\omega_{i}^{2}$, and six zero for near zero) eigenvalues, that correspond to overall rotations and translations. For the transition state, one Finds F - 1 positive eigenvalues that correspond to the $\omega_{i}^{\ddagger 2}$. The other nonzero eigenvalue is negative, which implies an imaginary frequency i lob. The presence of one imaginary frequency indicates th potential along one degree of freedon, the reaction coordinate, has the local form of an inverted parabola or barrier. (Actually, in the case of the reactant, exper imental information may be available on the frequencies, so that it may not be absolutely necessary to obtain the $\omega_{i}$ a priori.)

To obtain the required geometries, or:- notes that each is an extrenum on the potential surface, so that root searching techniques, coupled with information on the gradient of the potential, are required. The recently developed analytic gradient techniques ${ }^{19}$ facilitate the 
required wark. Also, if second derivative information is readily available, which will certainly be the case in the very near future ${ }^{20}$, a new approach to finding transition states, due to Cerjan and Miller ${ }^{21}$, may be used.

An interesting application of the theory is to the vinylidene isomerization co acetylene,

$$
\mathrm{H}>\mathrm{H}=\mathrm{C}: \rightarrow \mathrm{H}-\mathrm{C} \equiv \mathrm{C}-\mathrm{H} \text {. }
$$

Osamura and Schaefer 22 performed the required electronic structure calculations described above, at a rather high "level" (with the configuration interaction(CI) of single and double electronic excitations relative to the Hartree-Fock reference - see Ref. 22 for the details). Fig. I shows the three stationary point geometries and Table I gives the vibrational frequencies. As a check on the theoretical calculations, one may note that the theoretical geowetry for acetylene agrees to within 17 with the experimental geometry and the theoretical vibrational frequencies for acetylene are within $10 \%$ of the experimental frequencies. The classical barrier $v_{0}^{\neq} i s$ found to be $6 \mathrm{kcal} /$ mol relative to vinylidene, and acetylene is lower in energy than vinylidene by $40 \mathrm{kcal} / \mathrm{mol}$. Electronic structure barrier heights are usually too high and, upon further refinements of the basis set, the barrier usually decreases. Osamura and Schaefer therefore applied a correction to their bartier, that empirically corrects for some omissions in their calculacion. Their "besc" 
classical barrier is estimated to be $v_{0}^{\ddagger}=4 \mathrm{k}: \mathrm{al} / \mathrm{mol}$ (thus the zero point corrected barrier is found to be $v_{2 P}=v_{0}^{\neq}+\left(\sum_{i=1}^{F-1} \cdot h \omega_{i}^{\ddagger}-\sum_{1=1}^{F} h \omega_{i}\right) / 2$ $=4-1.8=2.2 \mathrm{kcal} / \mathrm{mol}$ ).

The tunneling probability was estimated from the Eckart barrier formula given in Ref. 11. The density of states for the reactant was estimated from the Whitton-Rabincvitch semiempirical formula ${ }^{15}$, although te should be noted that the simpler semiclassical expression ${ }^{15}$,

$$
\rho(E)=E^{F-1} /\left[(F-1) ! \prod_{i=1}^{i=F} \omega_{i}\right],
$$

could have been equally used, since numerical comparisons of the rate calculated with the above formula and the Whitton-Rabinovitch formula did not differ by more than a few percent.

Fig. 2 displays the rate constant results. The dashed curve is the rate calculated with the $6 \mathrm{kcal} / \mathrm{mol}$ barrier and the solid curve is the rate with the "best" barrier of $4 \mathrm{kcal} / \mathrm{mol}$. It is clear from Fig. 2 that the rearrangement rate is very high, and for excitation energies of less than I kcal above the zero-point energy of vinylidene, the rate to form acetylene is $\geqslant 10^{10} s^{-1}$. This is in harmony with the experimental work of Skell and co-workers ${ }^{23 a}$, which indicates a rearrangement rate of greater ctan $10^{10} \mathrm{~s}^{-1}$, and also with the experiments of Reiser and co-workers ${ }^{23 b}$, which failed to detect any evidence of vinylidene. Basicly, one does not observe vinylidene because ics lifetime, $\sim 1 / k \sim 10^{-10}-10^{-12} \mathrm{sec}$, is so short 
that it rearranges to acetylene before it has time to collide and react with other molecules (which would indicate its presence). The time between collistons in such experiments is $\sim 10^{-7} \mathrm{~s}$, i.e. much longer that the lifetime of vinylidene.

Before going on with soms other applications and extenstons, It should be noted that there is some uncertainty in these results due to uncertainty in the bartler height. As can easily be seen from Fig. 2, the rate is extremely sensitive to the value chosen for the barrier. In fact, Krishnan et al ${ }^{24}$ have challenged the present barrier, claiming that the zero-point corrected barrier is less than $1 \mathrm{kcal} / \mathrm{mol}$, and possibly even zero. This would imply that tunneling per se is not the reason for vinylidene being difficult to observe. Rather, because then even very small excitation energies are above the barrier, it is simply energetics, and not cunneling, that leads to the quick rearrangement to acetylene. Unfortunately, it is not clear at present which calculation is correct, since Krishnan et al ${ }^{24}$ base their conclusions on high level perturbation theory results and Osamura and Schaefer ${ }^{22}$ use a high level variational treatment - i.e. both methods have different strong and weak point.i. 
C. Reaction Path Hamiltanian and the HNC + HCN Reaction

C1. The Reaction Path Hamiltonian

In the previous section, the $F$ degrees of freedom of the transition scate were decomposed into a reaction coordinate, along with F - 1 bound vibrational degrees of freedom. What discinguished the reaction coordinate was that the frequency associated with it (obtained from a normal mode analysis at the transition state) was Imaginary, implying an inverted parabola or barrier potencial along its direction. This analysis was entirely local and only valid very close to the transicion state geometry. Naturalty, as the molecular coordinates change from this geometry, the frequencies associated with the F - 1 vibrational modes will change. Furthermore, they will, in general, be coupled to one another in some sort of dynamical fashion. In the limit of the reactant geometry, the frequencies of these modes will have hanged to cake on $F$ - I of the reactant mode frequencies, and the potential profile along the reaction coordinate will now appear bound, with frequency corresponding to the other reaceant mode. Similar arguments apply to the product limit. The effect of the changing frequencies and couplings between the modes can be incorporated into the calculation of the tunneling probabilities associated with a unimolecular reaction. In this section, a more rfgurous formulation of the reaction coordinate and the modes normal to 1 , valid at intermediate geometries, will be given and a classical Hamiltonian, the reaction path Hamiltonian of Miller, Handy, and Adams ${ }^{10}$. 
w111 be discussed.

Consider an $\mathrm{N}$ atom system with a transition state on its potential surface separating the relevent reactant and product regions. If $R_{i Y}$ denotes the Cartestan coordinates of the nuclei, with $i=1,2, \ldots, N$, and $Y=x, y, z$, it is usually most convenient to work in the mass weighted coordinates,

$$
x_{i Y}=\sqrt{m_{i}} R_{\perp Y},
$$

since then the masses $m_{i}$ do not enter into the equations explicitly. One now defines a curve through the $3 \mathrm{~N}$ dimensional coordinate space that passes through the reactant, transition state, and product geometries. Although there are many such curves, the most relevant is the reaction path, which is defined as the zero kinetic energy trajectory from the transition state down to the product and reactant regtons. In Appendix $A$ it is shown that if $s$ is the distance travelled along the reaction path, the associated mass - weighted Cartesian coordinates must satisfy

$$
\partial x_{i \gamma} / \partial s=-c \partial v / \partial x_{i \gamma} \quad,
$$

where $c^{-2}=\sum_{f \gamma}\left(\partial v / \partial x_{f Y}\right)^{2}$, and $v$ is the usual Born - Oppenhefmer potentlal energy. Thus, starting from the transition state, which will always be taken as $s=0$, one may solve the first order 
differential equations, eqs. II-5, to obtain $x_{i \gamma}(s)$, if $\partial v / \partial x_{i \gamma}$ can be readily evaluated.

We constrain the system to have zero total angular momentum so that there are $F=3 N-6$ relevent degrees of freedom (the six overall rotations and translations befing ignored). (me of the coordinates is chosen to be the reaction coordinate $s$. The other F - 1 coordinates then correspond to vibrational motion orthogonal to this reaction coordinate and are denoted by $Q_{k}$. Miller, Handy and Adams ${ }^{10}$ performed a canonical transformation from the usual Cartesian coordinates and momenta $\left(x_{i \gamma}, P_{i \gamma}\right)$ to these new coordinates and their associated momenta $\left(s, Q_{k}, P_{s}, P_{k}\right)$, which resulted in the Hamiltonian,

$$
\begin{gathered}
H\left(P_{s}, s, \underline{P}, Q\right)=\sum_{k=1}^{F-1}\left(\frac{1}{2} P_{k}^{2}+\frac{1}{2} \omega(s)^{2} Q_{k}^{2}\right)+v_{0}(s) \\
+\frac{1}{2}\left[p_{s}-\sum_{k, k^{\prime}=1}^{F-1} Q_{k} P_{k^{\prime}} B_{k, k^{\prime}}(s)\right]^{2} \\
{\left[1+\sum_{k=1}^{F-1} Q_{k} B_{k, F}(s)\right]^{2}}
\end{gathered}
$$$$
\text { I I-6 }
$$

where it has beerl assumed that for fixed $s$, the potential along each vibrational degree of freedom $Q_{k}$ is hamonic. $v_{0}(s)$ is the (nonharmonic) potential along the reaction coordinate, $w_{k}(s)$ are the harmonic frequencies associated with the $Q_{k}$ and the $B_{k k^{\prime}}(s)$ are elements 
which couple the various vibrational modes with one another $\left(B_{k F}\right.$ denotes the direct coupling of mode $k$ to the reaction cnordinate).

The frequencies $w_{k}(s)$ and coupling elements $B_{k k}$, in eq. II-6 are obtained as follows. The $3 \mathrm{~N}$ by $3 \mathrm{~N}$ force constant matrix $\underset{\approx}{\mathrm{K}}$ is defined to be the matrix of (mass-weighted) second derivatives,

$$
k_{i Y, i^{\prime} \gamma^{\prime}} \equiv \frac{\partial^{2} v}{\partial x_{i \gamma} \partial x_{i^{\prime} \gamma^{\prime}}} \text {, }
$$

evaluated at the conrdinates on th reaction path. The matrix is a matrix that projects onto the directions corresponding to the overall translations and rotations, as well as the reaction coordinate direction (which is proportionate to the gradient vector). The explizic form for $P$ is given in Ref. 10. The projected force constane matrix is then defined to be

$$
(1-P) \cdot k \cdot(\underline{z}-\stackrel{p}{z}, \underline{z},
$$

and has seven zero eigenvalues corresponding to the overall translations and rotations, as well as the reaction coordinate direction. The other $3 \mathrm{~N}-7=F-I$ nonzero eigenvalues ate the $w_{k}^{2}$, with associated eigenvectors denoted by $L_{i \gamma, k}(s)$. Let the normalized negative gradient rector be the $k=F$ eigenvector:

$$
L_{i \gamma, F}(s)=-c \partial V / \partial x_{i \gamma}
$$


The coupling elements are then given by

$$
B_{k, k^{\prime}}(s)=\sum_{i, \gamma} \frac{\partial L_{\left.i Y, k^{(}\right)}}{\partial s} L_{i \gamma, k^{\prime}}(s) \text {. }
$$

The terms $B_{k F}(s)$ couple the various normal modes to the reaction coordinate and are also related to the curvature $k(s)$ of the reaction path :

$$
K(s)=\left[\sum_{k=1}^{F-1} B_{k, F}(s)^{2}\right]^{\frac{1}{2}}=\left[\sum_{i, \gamma} x_{\dot{I} Y}^{\prime \prime}(s)^{2}\right]^{\frac{1}{2}},
$$

The harmonic oscillacor coordinates $\left(Q_{k}, P_{k}\right)$ are not always the most convenient ones. The action - angle variables $\left(n_{k}, q_{k}\right)$ corresponding to these coordinates are defined by 10

$$
\begin{aligned}
& Q_{k}=\sqrt{\left(2 n_{k}+1\right) / u_{k}} \sin q_{k}, \\
& P_{k}=\sqrt{\left(2 r_{k}+1\right) w_{k}} \cos q_{k},
\end{aligned}
$$

and will be useful in the percurbacive arguments that follow. The Hamiltonian in terms of these variables is given by

$$
\begin{gathered}
H\left(p_{s}, s, n, q\right)=\sum_{k=1}^{F-1}\left(n_{k}+\frac{1}{2}\right) w_{k}(s)+v_{0}(s) \\
\left.+\frac{\left[p-\sum_{k, k^{\prime}}=1\right.}{\left[1+\sum_{k=1}^{F-1} \sqrt{\left(2 n_{k}+1\right) / \omega_{k}(s)} \sin q_{k} B_{k, F}(s)\right] 2} \sin q_{k} \cos q_{k^{\prime}} B_{k, k^{\prime}}(s)\right]^{2}
\end{gathered}
$$


In eq. II-10 the diagonal elements $B_{k k}$ (s) are defined as

$$
B_{k, k}(s) \equiv-\frac{w_{k}^{\prime}(s)}{2 w_{k}(s)} \text {, }
$$

where here the prime denoces differentiation with respect to $s$. (Note that in the $Q_{k}, P_{k}$ Hamiltonian, eq. II-6, $B_{k k}=0$. )

C2. An Improved Rate Constant Formula

In sec. Cl, a more rigorous definition of the reaction coordinate and associated reaction pach Hamiltonian was given in which the frequencies of the normal modes change as $s$, the reaction coordinate, changes, and also certain couplings between the modes occur. It is possible to incorporace these effects into a calculation of the rate constant. This will be accomplished with the classical perturb ation theory described in Born's famous book ${ }^{25}$. The basic idea is to take the original Hamiltonian, in terms of the zero - order action angle variables $(e q . I I-10), H\left(p_{s}, s, n, q\right)$, and through certain canonical transformations arrive at yet another Hamiltonian, $H\left(P_{S}, S, N\right)=E$, which does not depend explicitly on the new angle variables conjugate to $N$. Effectively, for fixed $p_{S}$ and $s$, one has averaged over the $F$ - I vibrational modes. One then, in the spirit of the old quantum theory, associates the new actions $\mathbb{N}$ with the quantum numbers of the modes at the transition state and calculates a tuneling probability for each combination of $\mathrm{N}$ via the WKB approximation,

$$
I(E)=\Sigma_{N}\left[1+e^{2 \theta(E, N)}\right]^{-1}
$$


where $\theta$ is the $d i$ barrier penetration integral,

$$
\theta(E, N)=\int_{s}^{s} d_{s}^{s} \operatorname{Im} p_{s}(s, E, N)
$$

This is completely analagous with the simple treatment of sec. IIB, except that implicit in eqs. II-11 and II-12 are the (average) effects of changing frequencies and mode couplings. The microcarionical rate constant is then given as before (cf. eq. II-4).

The transformation $\mathrm{H}\left(\mathrm{P}_{\mathrm{s}}, s, \underset{\sim}{\mathrm{n}}, \underset{\mathrm{q}}{)}\right)+\mathrm{H}\left(\mathrm{P}_{s}, s, \underset{\sim}{N}\right)$ is very difficult to do exactly. We will carry it out to second order in perturbation theory, which, although not exact, suffices for the present application to hydrogen isocyanide rearrangement, since the effects to be included actually are small. (There are, of course, better, but more numerical ways of accomplishing such transformations ${ }^{26}$ and these could possibly be used for more pathological examples.)

Considering the coupling elements $B$ to be the perturbation, one can write the Hamiltonian as

$$
H\left(p_{s}, s, \underline{\sim}, q\right)=H_{0}\left(p_{s}, s, \underline{n}\right)+H_{1}\left(p_{s}, s, \underline{n}, \underline{q}\right)+H_{2}\left(p_{s}, s, \underline{n}, \underline{q}\right)
$$

where $H_{0}$ is given by

$$
H_{0}\left(p_{s}, s, \underline{n}, \underline{q}\right)=\sum_{k=1}^{F-1}\left(n_{k}+\frac{1}{)} \omega_{k}(s)+v_{0}(s)+\frac{1}{2} p_{s}^{2}, \quad I I-13 b\right.
$$

and $\mathrm{H}_{1}$ and $\mathrm{H}_{2}$ are given by 


$$
\begin{array}{cl}
H_{1}\left(p_{s}, s, n, q\right)=-p_{s} c_{1}-p_{s}{ }^{2} C_{2} & \text { II - 13c } \\
H_{2}\left(p_{s}, s, n, q\right)=\frac{1}{2} c_{1}{ }^{2}+\frac{3}{2} p_{s}{ }^{2} c_{2}{ }^{2}+2 p_{s} C_{1} C_{2} & \text { II-I3d }
\end{array}
$$

with

$$
\begin{gathered}
c_{1} \equiv \sum_{k, k^{\prime}} B_{k, k^{\prime}}(s) \sqrt{\left(2 n_{k}+1\right)\left(2 n_{k^{\prime}}+1\right) \omega_{k^{\prime}}(s) / \omega_{k}(s)} \sin q_{k} \cos q_{k^{\prime}} \\
c_{2} \equiv \sum_{k} B_{k, F}(s) \sqrt{\left(2 n_{k}+1\right) / \omega_{k}(s)} \sin q_{k} .
\end{gathered}
$$

Following Born ${ }^{25}$, classical perturbation theory then gives the Hamiltonian in terms of the good action-angle variables as a perturb ation series :

$$
E\left(p_{s}, s, N\right)=E_{0}\left(p_{s}, s, N\right)+F_{1}\left(p_{s}, s, N\right)+E_{2}\left(p_{s}, s, N\right)+\ldots \quad \text { II }-14 a
$$

where

$$
\begin{aligned}
& E_{0}\left(P_{s}, s, N\right)=H_{0}\left(P_{s}, S, \underset{N}{N}\right) \\
& \text { I I }-14 b \\
& E_{l}\left(p_{s}, s, N\right)=\bar{H}_{l}\left(p_{S}, s, N\right) \\
& I:-14 c \\
& E_{2}\left(P_{s}, s, \underset{\sim}{N}\right)=\bar{H}_{2}\left(P_{s} s, N\right) \\
& -\frac{1}{2} \sum_{k} \frac{k \cdot \frac{\partial}{\partial N}\left|\underline{k}(s)\left(p_{s}, s, N\right)\right|^{2}}{k \cdot w(s)} \\
& \text { I I }-14 d
\end{aligned}
$$


with

$$
\begin{aligned}
& \bar{H}_{i}\left(p_{s}, s, N\right)=(2 \pi)^{-(F-1)} \int_{0}^{2 \pi} d q H_{i}(p, s, N, q) \\
& H_{\underline{k}}(1)\left(p_{s}, s, \underline{N}\right)=(2 \pi)^{-(F-1)} \int_{0}^{2 \pi} d \underline{q} e^{-i k \cdot q} H_{1}\left(p_{s}, s, N, q\right) \quad I I-15 b
\end{aligned}
$$

Using eqs. II-14 and II-15, a straightforward calculation gives

$$
\begin{array}{cc}
E_{0}\left(P_{s}, s, \underset{\sim}{N}\right)=\frac{1}{2} P_{s}{ }^{2}+v_{0}(s)+\sum_{k}\left(N_{k}+\frac{1}{2}\right) \omega_{k}(s) & \text { II-16a } \\
E_{1}\left(P_{s}, s, N\right)=0 & \text { II-16b } \\
E_{2}\left(P_{s}, s, N\right)=A(s, N)+P_{s}{ }^{2} B(s, N)-P_{s}{ }^{4} C(s, N), & \text { II-16c }
\end{array}
$$

where

$$
\begin{aligned}
& A(s, N)=\frac{1}{2} \sum_{k<k^{\prime}} B_{k, k^{\prime}}(s)^{2}\left(N_{k}+\frac{1}{2}\right)\left(N_{k}^{\prime}+\frac{1}{2}\right) \frac{\omega_{k}(s)^{2}+w_{k^{\prime}}(s)^{2}}{w_{k}(s) w_{k^{\prime}}(s)} \\
& +\sum_{k}\left(\frac{\omega_{k}^{\prime}(s)}{4 \omega_{k}(s)}\right)^{2}\left(N_{k}+\frac{1}{2}\right)^{2} \\
& B(s, N)=\frac{3}{2} \sum_{k} B_{k, F}(s)^{2} \frac{N_{k}+\frac{1}{2}}{\omega_{k}(s)}-\frac{1}{\theta} \sum_{k} \frac{\omega_{k}^{\prime}(s)^{2}}{\omega_{k}(s)}{ }^{2}\left(N_{k}+\frac{1}{2}\right) \\
& -\frac{1}{2} \sum_{k<k^{\prime}} B_{k, k^{\prime}}(s)^{2} \frac{\left[\omega_{k}(s)\left(N_{k^{\prime}}+\frac{1}{2}\right)-\omega_{k^{\prime}}(s)\left(N_{k}+\frac{1}{z}\right)\right]}{\omega_{k}(s)^{2}-1 \omega_{k}(s)^{2}} \frac{\left[\omega_{k}(s)^{2}+\omega_{k}^{\prime}(s)^{2}\right]}{\omega_{k}(s) \omega_{k^{\prime}}(s)}, \\
& C(s, N)=\sum_{k} \frac{B_{k, F}(s)^{2}}{n_{k}(s)^{2}} . \\
& \text { II-16e } \\
& \text { II-16f }
\end{aligned}
$$


With the Hamiltonian expressed in terms of $\left(p_{s}, s\right)$ and the good action-angle variables, it is then easy to use energy conservation, i.e.,

$$
E_{0}\left(p_{s}, s, N\right)+E_{1}\left(p_{s}, s, \underline{N}\right)+E_{2}\left(p_{s}, s, N\right)=E
$$

to define Im $p_{s}(s, E, N)$. One finds

$$
\begin{gathered}
\operatorname{Im} P_{S}(S, E, N)=\sqrt{2(V-E+A)} \\
X\left[B+\frac{1}{2}+\sqrt{\left(B+\frac{1}{2}\right)^{2}+4 C(V-E+A)}\right]-\frac{1}{2}
\end{gathered}
$$

where $A, B, C$ are as defined in eq. II-I6 and

$$
v=v_{0}(s)+\sum_{k}\left(N_{k}+\frac{1}{2}\right) w_{k}(s)
$$

From eqs. II-17 one can caluclate the barrier penetration integraI via eq. II-12, the corresponding tunneling probability from eq. II-II, and finally the microcanonical rate from eq. II-4.

To summarize, the present model is vibrationally adiabatic in that the transformation to good action angle variables $(\underline{n}, q)+(\mathbb{N}, ?)$ is performed holding $P_{S}$ and $s$ fixed as parameters. This is an approximation. Also, the transformation was carried out to only second order perturbation theory and is thus only valid for small coupling perturbations. 
c3. Tunneling in $\mathrm{HNC}+\mathrm{HCN}$

The first step is to obtain the relevant portion of the reaction path and the corresponding frequencies and couplings. Yamaguchi and Schaefer ${ }^{27}$ performed the required electronic structure calculations within the self-consistent field (SCF) Hartree-Fock approximation (see Ref. 27 for details). They also performed a limited number of higher level (configuration interaction) calculations. They found, for example, that the geometry of the transition state predicted by the SCF theory was within $3 z$ of the more exact $C I$ result. The $C I$ barrier height (relative to HNC), however, was $36 \mathrm{kcal} / \mathrm{mal}$ compared to the SCF value of $40 \mathrm{kcal} / \mathrm{mol}$. Since tunneling is quite sensitive to the barrier height, the results we report will be as a function of the total energy relative to the barrier height, $E-v_{0}^{\ddagger}$, which should be somewhat insensitive to the choice of $v_{o}^{\ddagger}$.

To determine the reaction path $x_{i \gamma}(s)$, one starts at the transition stace and takes a step in the direction of the eigenvector associated with the imaginary frequency, $L_{i Y, F}(0)$ :

$$
x_{i \gamma}(\Delta s)=x_{i \gamma}(0) \pm L_{i Y, F}(0) \Delta s,
$$

where $\Delta s$ is an appropriate step size and the + or - takes one to one or the other side of the transition state. Subsequent steps are then determined from

$$
x_{i Y}\left(s_{j}\right)=x_{i Y}\left(s_{j-1}\right)+L_{i Y, F}\left(s_{j-1}\right) \Delta s,
$$


where $L_{i \gamma, F}(s)$ is the negative normalized gradient vector (cf. the r.h.s, of eq. (I-5). This approach is basicly Euler's method ${ }^{28}$ of solving the differential equations given by eqs. II-5, and represents the simplest way to find a reaction path. Unless the step size in s is chosen very small, however, the reaction path generated by the above approach will oscillate about the true path. Although there are ways to patch up this difficulty ${ }^{29}$, or avoid it ${ }^{21}$, we chose simply to smooth out the nonphysical ascillations "by hand", which should be satisfactory, since only a small portion of the reaction path near the transition state yields significant tunneling probabilities.

The relevant portion of the reaction path is shown in Fig. 3, where $s$ is reported in units of amu ${ }^{1 / 2}{ }_{A}\left(1\right.$ anu $\left.{ }^{1 / 2} a_{A}=80.679 \mathrm{~m}_{e}^{1 / 2} a_{o}\right)$. A step size of $\Delta s=0.1$ amu ${ }^{1 / 2} B$ was used in the calculations. The SCF force constant matrix was evaluated for $s=0, \pm 0.3$, and \pm 0.5 and ${ }^{1 / 20}$.

The coupling elements $\mathrm{B}_{\mathrm{kk}}$, and frequencies $w_{k}$ were obtained from the eigenvalues and eigenvectors of the projected force constant matrix (see eq. II-8). The $B_{k k}$, were obtained from eq. II-9 using finite differences to estimate the derivatives. Since $F=3$, there are only two frequencies, essentially the $H-(C N)$ stretch, and the $C-N$ stretch. The reaction coordinate $(k=F=3)$, is mostly the bending vibration. As was mentioned earlier, some CI calculations were also performed. Owing to the expense of such calculations, Yamaguchi and Schaefer were only able to calculate the CI force constant matrix at the transition state. To obrain the best estimates of the frequencies away from the transition state, $w_{k}(s)$, the SCF frequencies were 
scaled by $w_{k}^{C I}(0) / w_{k}^{S C F}(0)$. The potential $v_{0}(s)$ was itt to an Eckart function $^{18}$ with the $C I$ energetics and the CI imaginary frequency at s $=0$. Fig. 4 displays the relevant scaled frequencies and the potential $v_{0}(s)$. Fig. 5 displays the relevent couplings $B_{k k}$. $B_{12}$ couples the two modes orthogonal to the transition state to one another, ${ }_{13}$ couples the $\mathrm{H}-\mathrm{CN}$ stretch to the reaction coordinate, and the other coupling $B_{23}$, which couples the $\mathrm{CN}$ stretch to the reaction coordinate, was found to be small and essentially zero within the present numerical error $\left( \pm 10^{-3} \mathrm{~m}^{-1 / 2} \mathrm{a}_{\mathrm{o}}\right)$. It should be noted that these couplings are relatively small, especially in comparison with the curvature couplings in the $\mathrm{H}+\mathrm{H}_{2}$ problem ${ }^{10}$.

The microcanonical rate constant, $k(E)$, for the HNC $+\mathrm{HCN}$ reaction is shown in Fig. 6, as a function of the energy relative to the classical barrier height $\left(v_{0}^{\neq}=v_{s p}\right.$ in the figure). The classical threshold is denoted by an arrow. Below this energy the rate is seen to vary exponentially with the energy, a characteristic feature of tunneling. To see the importance of tunneling, one could note that an Ideal experiment, performed in a molecular beam, would be capable of measuring rates faster than $10^{5} \mathrm{~s}^{-1}$ ( the time of flight across a molecular beam chamber is $\sim 10^{-5} \mathrm{~s}$ ). Fig. 6 indicates that rates this fast occur for energies as low as $8 \mathrm{kcal} / \mathrm{mol}$ below tine classical threshold.

Finally, to assess the importance of fncluding the coupling elements $B_{k k}$, in the tunneling probability, the calculations were 
carried out with all the B's set to zero. This decreases the rate constant, but not by much; it is $5 \%$ smaller at $E-v_{0}^{\ddagger}=3 \mathrm{kcal} / \mathrm{mol}$, $10 \%$ smaller at $E-V_{0}^{*}=0$, and $15 \%$ smaller at $E-V_{0}^{*}=-2 k c a l / m o l$. Over the physically significant energy region, the tunneling is not influenced much by the coupling elements. This is primarily because the reaction path is relatively flat chroughout the relevent region (see Fig. 3). In the case of $\mathrm{H}+\mathrm{H}_{2}$, the reaction pach 10 was very curved in the region of the transition state, implying large and significant couplings $B_{k F}$.

D. Tunneling in the Unimolecular Decompostion of Formaldehyde D1. Preliminaries

The photodissociation of formaldehyde has attracted considerable theoretical and experimental interest in recent years ${ }^{30}$. Let us consider the collisionless limit of the process, with the following 1dealized sequence of events. First a laser electronically excites formaldehyde from its ground singlet $\left(S_{0}\right)$ state to a single ro vibrational stace of the first exciced singlet state ( $\left.S_{1}\right)$,

$$
\mathrm{H}_{2} \mathrm{CO}\left(\mathrm{S}_{0}\right)+h \omega+\mathrm{H}_{2} \mathrm{CO}^{\star}\left(\mathrm{S}_{1}\right), \quad \text { II- } 19 \mathrm{a}
$$

which then decays by either re-emitting a photon (fluorescence),

$$
\mathrm{H}_{2} \mathrm{CO}^{*}\left(\mathrm{~S}_{1}\right)+\mathrm{H}_{2} \mathrm{CO}\left(\mathrm{S}_{\mathrm{o}}\right)+\mathrm{hw}^{\prime},
$$


or by undergolng a radiationless transition to a highly vibrationally excited $s_{0}$ state,

$$
\mathrm{H}_{2} \mathrm{CO}^{*}\left(\mathrm{~S}_{1}\right) \rightarrow \mathrm{H}_{2} \mathrm{Co}^{\ddagger}\left(\mathrm{S}_{0}\right)
$$

which then may decompose unimolecularly,

$$
\mathrm{H}_{2} \mathrm{CO}^{\ddagger}\left(\mathrm{S}_{\mathrm{O}}\right)+\mathrm{H}_{2}+\mathrm{CO}
$$

The symmetry is such that molecular products $\mathrm{H}_{2}$ and $\mathrm{CO}$ only correlate with the ground $\mathrm{S}_{0}$ state. It has been assumed that the laser excltation energy is not too much above the $S_{0} \rightarrow S_{1}$ origin so that radical products ( $\mathrm{HCO}+\mathrm{H}$ ) are energetically inaccessible.

The experimental results ${ }^{30,31}$ are consistent with very fast nonradiative decay rates for $S_{1}$. Furthermore, it appears that this nonradiative decay is small when $\mathrm{D}_{2} \mathrm{CO}$ is used instead of $\mathrm{H}_{2} \mathrm{CO}$. A particularly attractive idea is, therefore, that tunneling 32,11 (in reaction II-19d) plays a significant role - i.e. that there is a very fast nonradiative decay from $S_{1}$ to $S_{0}$ that is coupled with tunneling to form molecular products.

Recently, Goddard, Yamaguchi, and Schaefer ${ }^{33}$ have performed extensive and highly accurate ab 1nitio contlguration interaction calculations on the $S_{0}$ surface, and in the subsequent section their results will be used (along with some supplementary calculations by 
Yamaguchi and Schaefer) to obtain accurate rate constants ${ }^{34}$ for reaction II-19d.

D2. Reaction Path and Tunneling Calculations

Tables II and III give the hamonic frequencies of stable ( $S_{0}$ ) formaldehyde and the harmonic frequencies and barrier height of the transition state of reaction II-19d, obtained from ab inftio calculations 33,34 at various levels of scphistication. The level of theory is increasing from left to right, with SCF denoting calculations made with a single (Hartree-Fock) configuration and CI denoting calculations with many (Iiterally tens of thousands) configurations. The reader may consult Refs. 32-34 for more cechnical details. The "experimental frequencies " of Table II are the harmonic frequencies inferred from experiment ${ }^{33}$. The "scaled frequencies" in Table ITI are the best estimates of the transition state frequencies, obtained by empirically modifying the DZP-CI frequencies by

$$
\left(\omega_{i}^{\text {scaled }}\right)_{\mathrm{TS}}=\left(\omega_{1}^{\mathrm{DZP}-C I}\right)_{\mathrm{TS}} \times\left(\omega_{1}^{\exp t I} / \omega_{1}^{\mathrm{DZP}-C I}\right)_{\mathrm{H}_{2} \mathrm{CO}}
$$

One point to be made about Tables II and III is that ab initio electronic structure theory estimates frequencies reascnably well, even at rather "low" levels of theory, such as the DZ-SCF level. Thus if one considers the RRKM rates predicted with the various levels of theory, and adjusts appropriately for the different barrier heights by, e.g., comparjng $k\left(E-v_{o}^{\ddagger}\right)$ rather than $k(E)$, 
there will not be much variation in the predicted rates. Using the RRKM + tunneling model described in sec. IIB, we obtained Table IV, which fndeed confirms these expectations. Thus for the energy region for which the rate constant is $10^{5}$ or larger, there is less than a factor of three variation in the rate constants predicted by the various levels of theory.

Fig. 7 shows the potential energy profile along the reaction path in the vicinity of the transition state, as given by the DZP-CI calculations. It has been previously determined that for these sort of tunneling calculations, one need only constder the region on either side of the transteion state to where the potential has fallen off to about $8 \mathrm{kcal} / \mathrm{mol}$ below its value at the transition state. The points in Fig. 7 are the ab initio values, and the solid Iine is an Eckart potentlal function ${ }^{18} \mathrm{flt}$ to the ab intlo energetics and curvature at the transition state. Thus $1 t$ appears that the Eckart potentlal form is well suited for this problem.

Ab initio force constant matrices were calculated at the D2P - CI level at values of the reaction coordinate $s= \pm 0.21$ and $0 \mathrm{amu}^{1 / 20}$; these are points at which $v_{0}(s)$ has fallen to about $8 \mathrm{kcal} / \mathrm{mol}$ below its value at the transition state. The frequencies and couplings were obtained as before (sec. IIC) from the projected force constant matrix. The frequencies are listed in Table $V$. Owing to cercain techni. 11 difficulties, the out-of-plane bend, $\omega_{6}$, was only estimated 
roughly (see kef. 33 for more explanation). However, since it is one of the lower frequencles, its varlation with $s$ is not expected to have a significant effect on the microcanonical rate. (But if the energy were not completely randomized, then it is possible shat certain mode specific effects, related explicitly to this out-of-plane bend, could play a role $\mathrm{e}^{36}$.)

The coupling elements $B_{k k}$ ' were rougly estimated using a finfte differences approximation to eq. II-9 and the three sets of elgenvectors obtained for $s= \pm 0.21$ and 0 . It w111 turn out that the actual effect of the coupling is small, so that a more accurate ietermination is unuecessary. Within the numerical error, the most slgnificant nonzero couplings may be considered constant over the $-c .21,0.21$ interval in $s$ and are Found to be $\mathrm{B}_{13} \cong 0.01, \mathrm{~B}_{34} \approx 0.015, \mathrm{~B}_{1 F} \cong 0.005$, and $\mathrm{B}_{3 F} \cong-0.009 \mathrm{~m}_{\mathrm{e}}^{1 / 2} \mathrm{a}_{0}$. The tunneling rates are presented in Table VI. Colum A is fust the simple RRKM + tunneling rate of sec. IIB, with the DZP-CI values for all relevent parameters. Column B is the same as A but with the Eckart potential replaced by the actual $v_{0}$ (s) points and a WTB approximation to the tunneling probability. Column $C$ Is the same as $B$, except that the frequenctes are allowed to vary along $s$ ( $1 . e$. the potential has the form of eq. II-17b), with each $\psi_{k}(s)$ being defined by a quadratic fit to the points in Table V. Column D includes the effect of coupling between the modes, using the perturbative formulae of sec. IIC. Colum E 
is the same as Column D, but with the couplings all multiplied by a factor of two, to see che effect of larger coupling.

One sees from Table VI how relatively insensitive the rate is to the level of rigour used to describe the tunneling dynamics. For example, the simplest model, Colum A, differs from the most rigorous model, Column $D$, by less than $20 \%$ for energies for which the rate is signiftcant $\left(\geqslant 10^{5} \mathrm{~s}^{-1}\right)$

Lec us now sumarize our best predictions of the microcanonical rate for reaction II-19d at the experimental excitation orjgin for $\mathrm{S}_{0}+\mathrm{S}_{1}$, hw $=80.6 \mathrm{kcal} / \mathrm{mol}$. The zero-point energy of formaldehyde is $16.8 \mathrm{kcal} / \mathrm{mol}$ (using the experimental Erequencies of Table II), so that the total energy is then $97.4 \mathrm{kcal} / \mathrm{mol}$. Using the best classical barrier of $92 \mathrm{kcal} / \mathrm{mol}$ we see that $E-v_{0}^{ \pm}=5.4 \mathrm{kcal} / \mathrm{mol}$. Interpolating from Column D, Table VI, and applying a factor of two due to symetry ${ }^{21}$, gives

$$
k=5.9 \times 10^{6} s^{-1}
$$

It is amusing to note that this terilt is in remarkable agreement with an earlier estimate of Miller, that used the simplest RRKM + tunneling theory and sove significantly different parateters.

This magnitude of $k$ near the origin of excitation has been shown $^{30}$ to be consistent with the experimental results, since it is significantly larger chan typical radiative decay rates $\left(\sim 2 \times 10^{5} \mathrm{~s}^{-1}\right.$ ). Also, if one replaces hydrogen by deuterium, the mass effect reduces 
$k$ by about a factor of ${ }^{11} 40$, makfing it comparable to or less than the radiative decay rates, which is agatn consistent with the experimental observations 30,31 .

However, this picture may not be perfect because there is still some uncertainty about the barrier hefght. Recent work by Frisch, Krishnan, and Pople 37 has suggested that the zero-point corrected barrfer is $V_{Z P}=80 \mathrm{kcal} / \mathrm{mol}$, which is to be contrasted with the best estimate of Goddard, Yamaguchi, and Schaefer ${ }^{33}, v_{Z P}=87 \mathrm{kcal} / \mathrm{mol}$. The result of Frisch et al is based on a perturbation theory approach that actually fncludes certain configurational effects not included by Goddard et al in their extensive many configuration variational calculation. On the other hand, the variational calculation includes more completely some configurational effects. Whatever the final outcome, one can still use Table VI to estimate the rate. Suppose, for example, that upon further refinements, the true zero-point corrected barrier 1 s found to be $83 \mathrm{kcal} / \mathrm{mol}$. Then the classical barrier is $83+16.8-11.8=88 \mathrm{kcal} / \mathrm{mol}$, where the zero-point energies have been taken out $(11.8 \mathrm{kcal}$ is the best estimate of the transitton state zero-point energy obtained from Table III). Thus $E-V_{0}^{\ddagger}=97.4-88=9.4 \mathrm{kcal}$. From Table VI, one incerpolaces $k=2 \times 10^{8} \mathrm{~s}^{-1}$, where again the factor of two due to symmetry ${ }^{11}$ has also been included. Here, we have used the previous observation that although the barrier height may vary, the rate as a function of $E-V_{0}^{\ddagger}$ remains relacively constanc. 
D3. A Closer Look at the Formaldehyde Decomposition Reaction Path Up until now, we have been only looking at a rather small portion of the reaction path that was important for tunneling. However, if one wishes to do a more detailed theoretical scudy, as, for example, in Ref. 36 where mode specific effects are considered, a wider and more detailed view of the path is necessary. To obtain such a view, including the frequency and coupling variations, would be exceedingly expensive with a high level of electronic structure theory. However, as has already been noted, the frequencies are reasonably well described with a relatively low level of theory, such as DZ-SCF.

In the following, we have used the computer code written by King, Dupuis, and Rys ${ }^{38}$ to obtain the reaction path, frequencies, and couplings at the simple $D Z-S C F$ ( $i$.e. one configuration HartreeFock) level.

The Reaction Path

The equilibrium and transition state geometries are both planar, and within the DZ-SCF level are 32
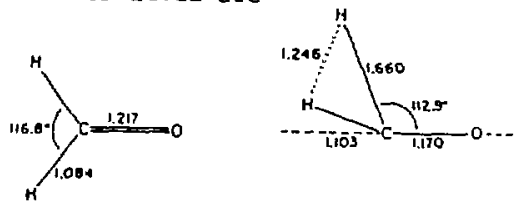

(with bond distances in $\AA$ ). As usual, the negative normalized gradient vector in mass-weighted coordinates (eq. II-5) was 
followed, with the initial step being in the direction of the eigenvector of the imaginary frequency at the transition state (see the procedure in sec. IIC). A step size $\Delta s=0.05 \mathrm{amu}^{1 / 2} \AA$ was found to be sufficient, and, owing to the steepness of the pocential, no nonphysical oscillations were apparent upon subsequent steps. (As a check, certain portions of the path were reproduced with a smaller step size of 0.025 amul/2.) A total of 28 steps were taken from the transition state towards equilibrium formaldehyde, and only 10 towards molecular products $\mathrm{H}_{2}+\mathrm{Co}$.

The reaction path is planar, since the gradient vector is always in the plane of the molecule. The corresponding potential profile is shown in Fig. 8. It will be noticed that the barrier is about $114 \mathrm{kcal}$ (but, if desired, could be readily scaled to whatever barrier height is desired). It was found that the reaction cooridinate (with imaginary frequency $2320 i$ at the transition state) corresponds to the $\omega_{6}\left(b_{2}\right)=1349 \mathrm{~cm}^{-1}$ mode in equilibrium formaldehyde. This is also evident from Fig. 8, since in the vicinicy of $\mathrm{H}_{2} \mathrm{CO}\left(\mathrm{s}=-1.4 \mathrm{amu}^{1 / 2} \AA\right)$, the potential indeed has the form $v_{0}(s) \approx(1 / 2) \omega_{6}\left(b_{2}\right)^{2} \Delta s^{2}$, i.e the curvature near equilibrium formaldehyde is less in absolute magnitude than the curvature of the potential at the transition state.

The Frequencies

As usual, the frequencies were obtained from the eigenvalues of the projected force constant matrix. This was done for a 
total of 30 geometries along the reaction path, allowing for a very clear view of how the frequencies change witlis.

There is a slight problem of nomenclature for the frequenctes. The standard numbering of the reactant frequencies, employed in the previous section in Table II is not in one to one correspondence with the standard numbering of the transition state frequencies in Table III. Thus, for example, the reaction coordinate frequency, as discussed above, corresponds to $\omega_{6}\left(b_{2}\right)$ of the equilibrium formaldehyde frequencles. This is not to be confused with the $w_{6}\left(a^{\prime \prime}\right)$ transition state frequency, which is really the out-of-plane bend. To represent the $w_{k}$ (s) we have chosen to use the labels of the transition state.

In Fig. 9 is shown the variation of the frequencies with s. There are some quite interesting features here. The two $\mathrm{CH}$ stretches ( $\omega_{1}$ and $\omega_{2}$ in the transition state labelling scheme) approach each other closely near equilfbrium formaldehyde and appear to have a sort of avoided crossing near $s=-1.1$. In the product limit, it appears that these two frequencles turn into the only two nonzero product frequencies, correspondirg to $\mathrm{H}_{2}$ and $\mathrm{CO}$. Notice also that the out-of-pla e bend, $w_{6}$, has a rather deep "well" near s $=-0.4$. The out-of-plane bend, by symmetry, does not couple with any of the other modes and thus may cross other frequencies, as it crosses $\omega_{4}$ near $s=-0.2$. The $\omega_{5}$ Frequency is abgent, since it is the reaction coordinate. The above features 
have been independently verified by Yamashita, Yambe, and Fukui ${ }^{39}$.

\section{The Coupling Elements}

As before, tive coupling elements were obtained with a finite difference approximation to eq. II-19. This approxtmation should be reasonably good here, stnce the spacting between points is now typically small $\left(0.05 \mathrm{amu}^{1 / 2} \AA\right)$. Fig. 10 ahows some of the couplings. An interesting observation, which holds for the other couplings, Is that $B_{21}$ ts large whenever the assoctated frequencies become close to each other. Thus $B_{21}$ ts quite large where the two $\mathrm{CH}$ stretches approach very closely, near $s=-1.1$. Generally speaking, FIg. 10 shows that the couplings can be quite large. However, it is amusing to note that in the vicinity of the transition state, the couplings are generally not too large. This is very fortunate, and indicates that the tunneling analysis discussed in the last section is reasonably justified.

As I final point, we may compare the couplings, obtained from many SCF calculations, to the couplings obtained in the previous section on the basis of on?y a few $C I$ points. We had previously found that the only significant couplings were approximately $\mathrm{B}_{13}=0.01, \mathrm{~B}_{34} \approx 0.015, \mathrm{~B}_{1 \mathrm{~F}} \approx 0.005$, and $\mathrm{B}_{3 \mathrm{~F}} \approx-0.009$. From the SCF calculattons, we find $B_{13} \approx 0.004, B_{34} \approx 0.02, B_{1 F} \approx 0.00$, and $\mathrm{B}_{3 \mathrm{~F}} \approx-0.009$, in satisfactory agreement, in view of the significant differences between the two calculations. 
E. Summary

In secs. IIA-IIC, tunneling in several unimolecular reactions was treated with varying levels of theoretica? sophistication.

In sec. IIA, the microcanonical rate constant for the isomerization of vinylidene to acecylene was predicted with a stmple RRKM + tunneling model due to M1ller ${ }^{11,22}$, and was found to be consistent with the avallable experimental evidence. The model used fgnored the dynamfcal effects of coupling between the various normal riodes with each other, and with the reaction coordinate. It is difficult to say whether or not the neglect of these effects is serious without doing a much more detailed study. Nonetheless the calculations reported suffice as a reasonable first guess.

In sec. IIB, a mre sophisticated dynamical model, based on the reaction path Hamiltonian ${ }^{10}$ was developed to account for the mode coupling effects. A combination of classical perturbation theory and the WKB method was used to obtain the tunneling probability and thus microcanonical rate constant. An application to hydrogen isocyanide fsomerization showed that the effects of coupling, at least for this reaction, are not very significant. Thus over the significant energy range, the coupling effects did not alter the rate by more than $15 \%$. The obvious reason for this lack of importance is the smallness of the coupling elements $B_{k k}$, in the reaction path Hamiltonfan formalism. Although in general it is difficult to predict a priorf if the coupling elements will be small, inspection of the reaction path shows that the reaction 
proceeds very smoothly, without any abrupt changes in the nature of the vibrational modes, implying that the reaction is relatively adiabatic. Furthermore, the reaction path itself has very little curvature in the region of the transition state, implying that the $B_{k F}$ which couple the vibrational modes to the reaction path are quite small. Not all reactions are this nice. In the case of $\mathrm{H}+\mathrm{H}_{2}$, the curvature effects are known to alter ther tunneling probabilicy by an order of magnitude or more ${ }^{10}$.

In sec. IIC the decomposition of formaldehyde was treated with the methods of secs. IIA and IIB. The rate constant obtained was found to be compatible with experiment. Again it was found that the effects of mode coupling play a relatively insignificant role, changing the overall rate by not more than 20\%. As with hydrogen isocyanide, the couplings in the region of the transition state were relatively small.

In sec. IID, however, a wider view of the formaldehyde reaction path was obtained. It was seen that although the couplings are not very large near the transition state, they can indeed become large along other parts of the reaction path. This suggests that a more detailed dynamical treatment (1.e. nonperturbative and nonstatistical) may indeed show that mode coupling is more important.

As pointed out earlier, the effect of tunneling in unimolecular reactions has not been treated very much in the literature (see forst ${ }^{17}$ for some brief remarks) and the applications presented here represent the Eirst serlous effort at illucidating its importance. 


\section{Charge Transfer from a Classical Perspective}

\section{A. Preliminaries}

One of the classic problems of chemical dynamics is collisional Ionization ${ }^{40}$, for example the reactions

$$
\begin{array}{cc}
\mathrm{Na}+\mathrm{I} \rightarrow \mathrm{Na}^{+}+\mathrm{I}^{-} & \text {III-1a } \\
\mathrm{Cs}+\mathrm{Br}_{2} \rightarrow \mathrm{Cs}^{+}+\mathrm{Br}_{2}^{-} & \text {III-Ib } \\
\mathrm{H}^{+}+\mathrm{H}_{2} \rightarrow \mathrm{H}_{2}^{+}+\mathrm{H} & \text { III-Ic }
\end{array}
$$

The problem is nonadiabatic in that at least two porential curves (or surfaces) must be considered, along with the associated couplings. Quantum mechanics provides the natural language for the description of such processes. However, glven a quantum mechanical Hamiltonian, tt is possible to work backwards, and obtaln a classical hamiltonian. Then, purely classical dynamics may be used to describe the problem. Such an approach has been adopted by McCurdy, Meyer, and Miller ${ }^{12}$ and has been successfully applied to the study of fine structure transitions in certain atom-atom and atom-molecule collisions. of course for reactions as simple as III-la, it is posstble to do an accurate quantum mechanical treatment ${ }^{41}$. However, for the related charge-transfer reactions such as III-1b and III-lc, an accurate quantum analysis is much more difficult, whereas the corresponding classical analogue approach to be described is still quite feastble.

Another classical approach to such problems, to be distinguished 
from the present, Is the surface-hopping trajectory method of Tully and Preston $^{42}$. In this approach, classical trajectories are followed on a single surface up to a crossing point, where then a hop to anothe: surface may take place according to a certaln probability. This method mixes both classical and quantum dynamics and, as pointed out by Millet ${ }^{43}$, can lead to a poor description of dynamical resonances. Nonetheless the Tully-Preston method has been quite successful in some applications, such as to reaction III Ic.

In the following, we will treat explicitly a two-state model of reaction III-la, although the method can be generalized easily to related reactions.

B. The Classtcal Analogue

In the quantum mechanical formulation of the problem, the Hamiltonian operator is sandwlched between elements of a complete and orthonormal set of basis functions to obtain an equivalent matrix representation. Commonly, two sorts of basis set are employed 44 . In one set, the potentfal terms are made diagonal, and in the other the kfnetic terms are made diagonal. These are called, respectively, adiabatic and diabatic representations. It is possible to obtain a classical analogue for elther case, but we consider here only the diabatic representation.

Considering a two-state model of the simplest charge transfer reaction, III-la, the quantum diabatic Hamiltonian representation of the stationary state Schrodinger equation is 


$$
\underset{\sim}{(T}+\underset{x}{V}(R)-\underset{z}{1} E) \psi_{\ell}(R)=0,
$$

where $\psi_{\ell}(R)$ is the $\ell^{\prime}$ th partial wave, $R$ is the internuclear distance, $E$ the total energy, and $(h=1)$

$$
\begin{aligned}
& \underset{\approx}{\mathrm{T}}=\underset{\approx}{1}\left(-(2 \mu)^{-1} \mathrm{~d}^{2} / \mathrm{dR}^{2}+\ell(\ell+1) / 2 \mu \mathrm{R}^{2}\right) \\
& \underset{\approx}{\mathrm{v}}=\left(\begin{array}{ll}
\mathrm{v}_{00} & \mathrm{v}_{01} \\
\mathrm{v}_{10} & \mathrm{v}_{11}
\end{array}\right),
\end{aligned}
$$

with $\mu=\mathrm{m}_{\mathrm{Na}} \mathrm{m}_{\mathrm{I}} /\left(\mathrm{m}_{\mathrm{Na}}+\mathrm{m}_{\mathrm{I}}\right)$. For the present application, $\mathrm{v}_{00}(\mathrm{R})$ may be considered a covalent Na-I interaction and $v_{11}(R)$ the ionic $\mathrm{Na}^{+}-\mathrm{I}^{-}$interaction. In the diabatic representation $\mathrm{V}_{00}$ and $\mathrm{V}_{11}$ may cross, but the potential matrix is nondiagonal. Had the adiabatic representation been employed, a noncrossing rule would hold, $\stackrel{\underline{V}}{\underline{y}}$ would be diagonal, and the coupling terms would be in the kinetic energy ${ }^{44}$.

By expanding $\stackrel{v}{\sim}$ in terms of Pauli spin matirces and then representing the spin operators in terms of classical action-angle varfables, Meyer and Miller ${ }^{12 c}$ showed that the corresponding classical analogue of the matrix Hamiltonian above is

$$
\begin{aligned}
\mathrm{H}_{\mathrm{cl}}(\mathrm{P}, \mathrm{R}, \mathrm{n}, \mathrm{q})=\mathrm{P}^{2} / 2 \mu & +\ell^{2} / 2 \mu \mathrm{R}^{2}+(1-n) v_{00}+n V_{11} \\
& +2 \mathrm{v}_{01} \sqrt{(\mathrm{n}+1 / 2)(3 / 2-\mathrm{n})} \cos q .
\end{aligned}
$$

The idea now is that the electronic degree of freedom is represented 
by the action-angle variables $(n, q)$. A value of $n$ near zero will correspond to mostly covalent states, whereas a value of $n$ near one will correspond to mostly fonfc states. (There are other ways of obtalning the classical Hamiltonian ${ }^{12}$, but for the two-state model the spin analogy is the simplest.)

C. Classical Trajectories for $\mathrm{Na}+\mathrm{I}+\mathrm{Na}^{+}+\mathrm{I}^{-}$

The diabatic matrix elements of Faist and Levine ${ }^{41}$ were employed In a relatively standard quastclassical trajectory study ${ }^{45}$ with the classical Hamlltonian of eq. III-2. For various fixed centre-of-mass energies $E$, the Initial positions and momenta were taken as $R_{0}=160 \AA$, $\underline{p}_{0}=-\sqrt{2 \mu E}, n_{0}=0$, and $q=2 \pi \zeta$, where $\zeta$ is a random number between 0 and 1. It was also necessary to average over the orbital angular momentum $l$, which is related to the classical impact parameter $b$ through $\ell^{2}=2 \mu \mathrm{Eb}^{2}$. We adopt the usual quasiclassical procedure ${ }^{45}$ of choosing $b$ from $b=\sqrt{\zeta^{\top}} b_{\max }$, where $\zeta^{\prime}$ is another random number between $O$ and 1 . The value of $b_{\max }$ must be such that no charge transfers will take place for larger values of $b$. A value of $9 \AA$ was found satisfactory (Initial and final electronic ac' sns were the same to three signficant figures). It will be noticed that the initfal internuclear separation (the "practical" asymptotic limit) is rather large (160A). This is because b is typically large for such charge transfer reactions (crudely, one could imagine many charge transfers taking place near the diabatic curves' crossing point of about $7 \AA-$ see, e.g., the "harpoon mechanism"l), and thus the centrifugal term $\ell^{2} / 2 \mu R^{2}=E b^{2} / R^{2}$ can be significant even for seemingly large values of 
the internuclear separation R. A standard (variable step-size, $5^{\prime}$ th order predictor-corrector) routine was then used to integrate Hamilton's equations,

$$
\begin{aligned}
& \dot{\mathrm{P}}=-\partial \mathrm{H}_{\mathrm{cl}} / \partial \mathrm{R} \\
& \dot{\mathrm{n}}=-\partial \mathrm{H}_{\mathrm{cl}} / \partial \mathrm{q} \\
& \dot{\mathrm{R}}=\partial \mathrm{H}_{\mathrm{cl}} / \partial \mathrm{P} \\
& \dot{\mathrm{q}}=\partial \mathrm{H}_{\mathrm{cl}} / \partial \mathrm{n},
\end{aligned}
$$

with $\mathrm{H}_{\mathrm{cl}} \mathrm{g}^{\prime}$ en by $\mathrm{eq}$. III-2, unt1l $\mathrm{R}$ was suffictently large $(\geqslant 15 \AA)$, and $P$ was $P$ sitive. A crude 'blming' procedure was used to determine the cross s ction for charge transfer. If the final action $\mathrm{n}_{\mathrm{f}}$ is between $1 / 2$ and $3 / 2$, then the trajectory is $t$-rned reactive. (There are better ways of analysing the products, such as the moment method ${ }^{46}$.) The charge ransfer cross section is then given by the well-known ..onte-Carlo formula ${ }^{45}$,

$$
\sigma(E)=\pi b_{\max }^{2} N_{r} / N
$$

where $N_{x}$ is the number of charge transfers and $N$ the number of trajectorle run at the energy E. The Monte-Carlo error, that is one standar deviation, is given by 45

$$
\delta \sigma=\sqrt{\left(\mathrm{N}-\mathrm{N}_{\mathrm{r}}\right) / \mathrm{N}} \cdot \overline{\mathrm{N}}_{\mathrm{r}} \sigma
$$


About 400 trajectories were run at each energy, with the corresponding Monte-Carlo error in the cross sections being about $10 \%$.

The results of the calculations are shown in Fig. 11. For comparison, the essentially exact quantum mechanical results of Faist and Levine 41 are shown as the solid curve. It is clear that the present classical nodel is qualitatively correct, and somewhat quantitative. It is conceivable, therefore, that the classical analogue approach might be useful for studying more complex charge transfer reactions, such as III-lb and III-lc, where it is difficult to obtain the quantum result.

D. A Semiclassical Model

The encouraging results of the previous section suggest that a sexiclassical model, based on the classical analogue Hamiltonian, might be appropriate. We follow here the semiclassical perturbation theory approach of Mfller and Smith ${ }^{47}$. The classical analogue Hamiltonian is written as

$$
H=H_{0}+H_{I} \text {, }
$$

where

$$
H_{0}=P^{2} / 2 \mu+\ell^{2} / 2 \mu R^{2}+(1-n) v_{00}+n V_{I 1}, \quad \text { III-3b }
$$

and

$$
H_{1}=\operatorname{Lr}(n+1 / L)\left(\overline{j / L-n)} v_{01} \cos q .\right.
$$


The semiclassical $S$ matrix for a transition $n_{1} \rightarrow n_{2}$ is then given approximately by 47,48

$$
s_{n_{2}+n_{1}}=(2 \pi)^{-1} \int_{0}^{2 \pi} d \bar{q} e^{-i \Delta n \bar{q}} e^{-i A}
$$

with

$$
A=\int_{-\infty}^{\infty} d t H_{1}(t)
$$

In eq. III-4, $\bar{q}$ is the initial value of the angle variable associated with the electronic action. To find the time dependence of $\mathrm{H}_{1}$, one assumes that it arises out of the zero-order trajectory determined by eq. II -3b:

$$
\begin{aligned}
& \dot{q}=v_{11}-v_{00} \\
& \dot{R}=\sqrt{\alpha\left(E-\ell^{2} / 2 \mu R^{2}-(1-n) v_{00}-n V_{I I}\right)} / \mu \\
& \dot{n}=0 \\
& \dot{P}=Q^{2} \cdot u R^{3}-(1-n) \partial v_{00} / \partial R-n \partial v_{11} / \partial R \text {. }
\end{aligned}
$$

The value of $n$ in eq. III-5, which is constanc to zero order, is caken as $\left(n_{1}+n_{2}\right) / 2=1.2$. As discussed by Miller and Smith ${ }^{47}$, this is one way of symmet.izing the $S$ matrix (but not the only way). The time evolution of $q$ is given by

$$
q(t)=\bar{q}+\int_{t_{0}}^{t}\left(v_{11} v_{00}\right) d t=\bar{q}+\int_{t_{0}}^{t} \omega d t \text {, }
$$


with $w=v_{11}-v_{00}$. Inserting this explicit (but approximate) time dependence for $q(t)$ into eq. III-4b, and changing integration varlables from $t$ to $R\left(d t=\mu P^{-1} d R\right)$ results in

$$
\begin{aligned}
& A=2 \mu\left[\int_{\infty}^{R_{t}} v_{01}\left(\cos \bar{q} \cos \int_{R_{t}}^{R} P^{-1} \mu \omega d R^{\prime}-\operatorname{sinq} \sin \int_{R_{t}}^{R} P^{-1} \mu \omega d R^{\prime}\right) P^{-1} d R\right. \\
& \left.+\int_{R_{t}}^{\infty} v_{01}\left(\cos \bar{q} \cos \int_{R_{t}}^{R_{P^{-1}}} \mu \omega d R^{\prime}-\sin \bar{q} \sin \int_{R_{t}}^{R} P^{-1} \mu \omega d R^{\prime}\right) P^{-1} d R\right], \text { III-6 }
\end{aligned}
$$

where $P= \pm \sqrt{2 \mu\left(E-l^{2} / 2 \mu R^{2}-\left(v_{00}+V_{11}\right) / 2\right)}$, and $R_{t}$ is the turning point ( $R$ value s.t. $P=0$ ).

In the first integral of eq. III-6, one sets $P<0$, corresponding to an incoming trajectory, and in the second integral $P=0$, which corresponds to an outgolng trajectory. This then leads to

$$
A=2 \cos \bar{q}
$$

where

$$
Z=4 \mu \int_{R_{t}}^{\infty} v_{01} \cos \int_{R_{t}}^{R} P^{-1} \mu \omega d R^{\prime} P^{-1} d R
$$

Eq. III-4a for the $S$ matrix then becomes

$$
\begin{aligned}
S_{n_{2}+n_{1}} & =(2 \pi)^{-1} \int_{0}^{2 \pi} d \bar{q} e^{-i \Delta n \bar{q}} e^{-12 \cos \bar{q}} \\
& =J_{\Delta n}(z)
\end{aligned}
$$


where $J_{\Delta \mathrm{n}}$ is a regular Bessel function of order $\Delta \mathrm{n}$. In the case of charge transfer, one has simply

$$
P_{1+0}=\left|s_{1+0}\right|^{2}=J_{1}^{2}(z)
$$

The argument $Z$ may be evaluated along the zero-order classical trajectory with eq. III-8. Numerical difficulties may arise (since $P=0$ at $R=R_{t}$ ) and it may be best to do the integral directly in the tine representation. In this case,

$$
z=4 \int_{0}^{\infty} d t v_{01}(t) \cos \int_{0}^{t} \omega\left(t^{\prime}\right) d t^{\prime},
$$

where the zero of time is understood to be the classical turning point $R_{t}$. The time dependence of $V_{O 1}$ and $w$ artses from eqs. II-5. An even simpler time dependence would be to assume a straight line trajectory so that

$$
R(t)=\sqrt{b^{2}+(P / \mu)^{2} t^{2}} .
$$

To fllustrate this model, let us consider the Landau - Zener 49 model,

$$
\begin{aligned}
& v_{00}=-F_{0}\left(R-R_{x}\right) \\
& v_{11}=-F_{1}\left(R-R_{x}\right) \\
& v_{01}=\text { constant }
\end{aligned}
$$


In addition, it will be assumed that the collision is at zero Impact parameter and that the nuclear velocity is constant. The zero-order motion is then

$$
\begin{aligned}
& P(t)=P \\
& R(t)=n=1 / 2 \\
& R(t)=P t / \mu+R_{x} \\
& q(t)=P\left(F_{0}-F_{1}\right) t^{2} / 2 \mu+\bar{q},
\end{aligned}
$$

where $t=0$ has been chosen to correspond to the crossing point $R_{x}$. We now have

$$
H_{1}(t)=2 v_{01}\left[\cos \bar{q} \cos P\left(F_{0}-F_{1}\right) t^{2} / 2 \mu-\sin \bar{q} \sin P\left(F_{0}-F_{1}\right) t^{2} / 2 \mu\right],
$$

and thus

$$
A=\int_{-\infty}^{\infty} d t H_{1}(t)=2 v_{01} \sqrt{\pi \mu / P\left(F_{0}-F_{1}\right)}(\cos \bar{q}-\sin \bar{q}) .
$$

The semiclassical $S$ matrix is then given by

$$
s_{n_{2}+n_{1}}=(2 \pi)^{-1} \int_{0}^{2 \pi} d \vec{q} e^{-1 \Delta n \vec{q}} e^{-1 Z(\cos \bar{q}-\sin \bar{q}) / \sqrt{2}},
$$

with

$$
z=2 \sqrt{2} v_{01} \sqrt{\pi \mu / F\left(F_{0}-F_{1}\right)}
$$


The probability of a transition then reduces to

$$
P_{1+0}=\left|s_{1+0}\right|^{2}=J_{1}^{2}(z),
$$

where the identity

$$
J_{n}\left(\sqrt{a^{2}+b^{2}}\right) e^{i n \alpha} \equiv(2 \pi)^{-1} \int_{0}^{2 \pi} d x e^{i[a \sin x+b \cos x]} e^{-\ln x},
$$

with $\alpha=\tan ^{-1}(b / a)$, has been used.

The correct quantum mechanical resuit for the Iandau-Zener model is ${ }^{49} P_{1+0}=1-e^{-z^{2} / 4}$. It is thus evident that in the Iimit $z \rightarrow 0$ both the approximate semiclassical result, eq. III-14, and the exact quantwm result agree, stnce then $J_{1}(z) \rightarrow z / 2$, and both formulae give $\mathrm{P}_{1+0} \rightarrow 2^{2} / 4$. Table VII gives a more detafled comparison of the semiclassical and quantum results. For small arguments $Z$, i.e. large velocities and/or small slope differences $\left(F_{0}-F_{1}\right)$, the semiclassical result is reasonable. It is interesting to note that 1 gives better results than both the standard classical trajectory method and primative semiclassical mechanics in this regime (see Ref. $12 b$ for the classical and primative semiclassical results). As might be expected, however, the approach fafls for larger arguments $z$. In this regtme, the semficlassical perturbation theory is Inspplicable (even probability conservation, $P_{0+} 0^{+} P_{1+}=1$, breaks down as $Z$ becomes large). In fact, by inspection of eq. III-14, one sees that the maximum value that $P_{1+0}$ can take on is about 0.35 . 
since that is about the largest value that $\mathrm{J}_{1}^{2}$ can cake on. Nonetheless, for the range $z \leqslant 1$, the perturbative model does seem adequate. Thus, if one does not have a simple linear approximation to the diabatic matrix elements, so that the Landau-Zener model is not directly applicable, one could perhaps use eqs. III-10 and III-11 to obtain the nonadiabatic transition probabilities in the limit of small arguments $Z$.

We now apply this semiclassical model to the $\mathrm{Na}+\mathrm{I}$ problem. One should keep in mind that this should be a rather severe cest since the charge transfer probabilities are quite high here and the semiciassical model is really only valid when the probabilities are small. The charge transfer cross section is given by

$$
\sigma(E)=2 \pi \int_{0}^{\infty} b d b P(b),
$$

with $P(b)=J_{1}^{2}(Z)$, with 2 given by $Q q$. II-ll. The $R(t)$ dependence, in principle, should be obtained by solution of eqs. III-5. However, for the present application we are only concerned with the high energy regime, where the semiclassical model is most valid. In this regime, the simple straight line trajectory approximation should be good, $R(t)=\sqrt{b^{2}+(P / \mu)^{2} t^{2}}$.

A simple trapazoidal rule incegration was performed over $b$ to ohtain $\sigma$, and each $Z(b)$ was also obtained by a trapazoidal tule integration of eq. III-11. The result is shown as a dashed line in Fig. 11. As expected, the model is adequate for the very high 
energles, but quite poor for the lower energies. For other sorts of two state processes, that occur with lower probabilities, one might expect this model to be more applicable.

\section{E. Concluding Remarks}

The success of the classical trajectory analysis of sec. IIIC suggests that the classical analogue might be useful for studying more complex sorts of charge transfers, such as $M+X_{2}+M^{+}+X_{2}{ }^{-}$, where $M$ is an alkali atom and $x_{2}$ a halogen molecule ${ }^{40}$. These sorts of reactions have been treated with Tully-Preston surface hopping approaches with some success, and it would be interesting to see if the classical analogue would be as useful. It may also be inceresting to examine $\mathrm{H}^{+}+\mathrm{H}_{2} \rightarrow \mathrm{H}_{2}^{+}+\mathrm{H}$, which also involves exchange of an $\operatorname{acom}^{42}$.

The semiclassical perturbation theory of sec. IIID is less generally applicable, since it is only valid in the case of suall ccupling and transtion prcbabilities (i.e. high energy). However, in this limit it may be useful since, unlike the Landau-Zener model 49 , for example, it can be used with any desired forms for the matrix elements $v_{00}, v_{11}$, and $v_{01}$. 


\section{Classical Aspects of the Laser Excitation of Molecules}

\section{A. Overview}

With the development of high-powered lasers, physical chemists have been exploring the nature of multiphoton and overtone absorption in molecules 50 . There is a growing need for simple theoretical models to incerpret and predict the results of such experiments. However, the correct theoretical treatment, a detailed solution of the Schrodinger equation, including the molecule-radiation interaction, is still out of reach for most systens of chemical interest. Many theoreticians have thus turaed to a classical description of the process $^{51}$, which is at least more tractable. There are then two obvious questions that must be answered. First, how realistic is the classical description? In sec. B we answer this question for a vibrating and rotating diatomic in a strong laser, since in this case it is also possible to obtain an accurate quantum solution. The second question that arises is, given that a classical description is correct to some extent, what is the nature of such a solution? We answer this question in sec. C for a model nonrotating diacomic in a strong laser by looking carefully at how classical states evolve with time, using a novel period advance map method ${ }^{13}$ to generate the solution. A very simple model, based on a pendulum ${ }^{14}$, is then invoked to explain 
many of the classical features, which include spirals in phase space.

B. Classical and Quantum Mechanical Studles of HF in an Intense Laser Field.

\section{B1. Preliminaries}

The simplest molecule-laser problem is a diatomic molecule Interacting with a strong 1aser. Accurate, numerical classical and quantum solutions may be obtained for this problem. Because of the small number of quantum states involved, this represents a particularly severe test of classical mechanics. Previously, Walker and Preston 52 have performed both quantum and classical calculations for a model, nonrotating HF molecule. Their results, with laser Intensitles $\geq 10 \mathrm{TW} / \mathrm{cm}^{2}$ (1TW $=10^{12}$ Watts), Indicated good agreement between classical and quantum predictions of energy absorption averaged over laser pulse times, except near multiphoton resonances. Vartous other aspects of the problem of a diatomic interacting with a laser have been examined using either classical 53 or quantum 54 models.

In this section, we examlne the detailed behavior of a vibrating and rotating diatomic molecule $f_{i}$ an intense laser field, and the valfdity of classical mechanics to describe this problem. The classical and quantum equations of motion are solved numerically for both rotating and, for comparison, nonrotating models of HF, inftially in 1 ts ground state. Laser intensities of 1.0 and $2.5 \mathrm{TW} / \mathrm{cm}^{2}$ 
are used, with frequencies in the range of the fundamental transition. Energy absorption and transition probabilities are calculated as a function of pulse time, as well as the pulse averaged absorption. Most of the work is for maximum pulse lengths between 0.9 and $2.0 \mathrm{ps}$ (between 100 and 250 optical cycles), although it was necessary to integrate the quantum solution near multiphoton resonances for much longer times. It is found that classical mechanics does not correctly describe the time behavior of the system. Furthermore, classical rotational state distributions are completely incorrect. -lassical mechanics, however, does give the correct magnitude of pulse averaged energy absorption. In addition, classical mechanics correctly indicates the presence of increased multiphoton absorption for frequencies lower than the one-photon resonance, although, in agreement with Walker and Preston's results 52 , specific resonance peaks are not resolved and only a small amount of multiphoton absorption occurs. The effect of laser phase, which is often neglected, is also studied and found to be only a swall effect on the quantum results and little or no effect on the classical results.

\section{B2. Methods}

\section{Classical Mechanics}

The Hamfltonian for a vibrating and rotating diatomic molecule, with reduced mass $\mu$, in spherical coordinates $r, \theta$ and $\phi$ is

$$
H_{0}=\frac{P_{\tau}^{2}}{2 \nu}+\frac{1}{2 u \tau^{2}}\left(P_{\theta}^{2}+P_{\phi}^{2}\left(\sin ^{2} \theta\right)+V(r) . \quad I V-1\right.
$$


where $P_{r}, P_{\theta}$, and $P_{\phi}$ are momenta canonically conjugate to $r, \theta$ and $\phi$, and $V(r)$ is the Barn-Oppenheimer potential function. In the absence of external fields, there are three conserved quantities which at the vibracional action,

$$
N_{v}=-\frac{1}{2}+\frac{1}{2 \pi} f p_{\tau} d r
$$

the rotational angular momentum,

$$
\sqrt{J(J+h)}=\left(P_{\theta}^{2}+\frac{P_{\phi^{2}}}{\sin ^{2} \theta}\right)^{2},
$$

and the $z$ projection of the angular momentum $M=P_{\phi}$. If an oscillating electric field of frequency $\omega, z$ polarizacion, and phase $\delta$ is incroduced, the Hamiltonian becomes

$$
H=H_{0}-d(r) \cos \theta E_{0} \sin (\omega t+\delta) \quad
$$

where $d(r)$ is the molecular dipole function and $E_{0}$ is the field strength, which is related to the intensity by ${ }^{55} E_{0}=(B \pi I / c)^{1 / 2}$. Eq. IV-2 is valid in the limit of high photon density, which is certainly true in the present study. For lower intensities, the classical formalism developed by Miller ${ }^{56}$ could be used.

With the interaction present, the vibrational action $\mathrm{N}_{\mathrm{V}}$ and rotationa! angular momentum $J$ are no longer conserved. However, with the present choice of polarization, $M$ is still conserved since $H$ has no $\$$ dependence. The complete classical solution involves 
specification of the appropriate initial conditions and solution of Hamilon's equations :

$$
\begin{aligned}
& \dot{p}_{t}=-\frac{\partial H}{\partial r}=\frac{1}{\mu r^{3}}\left(p_{\theta}^{2}+p_{\phi}^{2} / \sin ^{2} \theta\right)-\frac{\partial V}{\partial r}+\frac{\partial d}{\partial r} \cos \theta E_{0} \sin (\omega r+\delta) \\
& \dot{p}_{\theta}=-\frac{\partial H}{\partial \theta}=p_{\phi}^{2} / \mu r^{2} \sin ^{3} \theta-d(r) \sin \theta E_{0} \sin (\omega r+\delta)
\end{aligned}
$$

$\dot{\tau}=\partial H_{\partial} / \partial_{r}=p_{r} / \mu$

$\dot{\theta}=\partial H / \partial P_{\theta}=P_{\theta} / \mu r^{2}$

Approximate analycic orbits have been obtailed 57 for a rotating Morse oscillacor with no external field, and chese are used to determine the diatomic initial conditions (see Apperdix B for derails). This approximation is cellent for the $\mathrm{vj}^{+}$cation-rotation states of importance here. The laser phase $\delta$ is also averaged over in most cases ( $i . e$ each trajectory has $\delta$ chosen rar.domly between 0 and $2 \pi$ ), although it will be shown to be unimportant.

The energy absorbed as a function of pulse iengch is defined by

$$
\langle E(c)\rangle_{C L}=\frac{1}{N} \sum_{i=I}^{N} H_{0}\left(p_{r}{ }^{i}(0), p_{\theta}^{i}(0), r^{i}(0), \theta^{i}(0), \delta^{1} ; t\right)-E_{1} \quad I V-4
$$

where $N$ is the number of trajectories and $E_{1}$ is the initial nolecular eneray. which is the present study is the ground state $(v=0, j=0)$ energy. 
The final vibrational action $\mathrm{N}_{\mathrm{V}}$ after a pulse length $\mathrm{t}$ is also calculated with the rotating Morse oscillator approximation 57 . $J$ is calculated directly from $J(J+h)=p_{\theta}^{2}+p_{\phi}^{2} / \sin ^{2} \theta$. (Note: $P_{\phi}=0$ here since $J=0$ inicially.) With $h=1, N_{v}$ and $J$ are boxed according to the nearest integers $v, j$ such that $v-I / 2 \leqslant N_{v}$ $\leq v+1 / 2$ and $j-1 / 2 \leq J \leq j+1 / 2$, which is the usual quasiclassical quantization procedure. The transition probability into a particular $v, j$ state, as a function of pulse length is

$$
P_{v, j}^{C L}(t)=N_{v, j}(t) / N
$$

where $N_{v, j}(t)$ is the number of trajectories at time $t$ with final actions in the $v, j$ box.

of course, a single trajectory integrated out to some large pulse length $\mathrm{T}$ contributes to all intermediate pulse time results. Similar to Walker and Preston 52 , the pulse averaged energy as a function of laser frequency $w$ is defined as

$$
\tilde{E}_{C l}(w)=\frac{1}{T} \int_{0}^{T}\langle E(t)\rangle_{C L} d t \quad . \quad I V-6
$$

For comparison, nonrotating calculations were also performed. These calculations were done essentially in the same manner as described by Walker and Prescon 52 . More technical decails will be discussed later. 
Quantum Mechanics

Al hough Leasure, Milfeld, and Wyate ${ }^{54}$ tave developed an efficient means of determining the long time solution, the time scale of interest here is short enough (usually less than $20 \mathrm{Fs}$ ) that direct integration of the coupled quantum equations is possible.

The total wavefunction is expanded as

$$
\Psi_{m}(r, \theta, \phi, \tau)=\Sigma_{v, j} C_{v j m}(t) \chi_{v j m}(r, \theta, \phi)
$$

with

$$
X_{v j m}(r, \theta, \phi)=R_{v}(r) Y_{j n}(\theta, \phi) / r
$$

The $Y_{j m}$ are spherical harmonics and $R_{v}$ are Morse eigenfunctions 58 . Strictly speaking, $R_{v}$ should also depend on $j$, but in the present problem, with only small values of $j$ being important, such rotational corrections should be small. As in classical mectianics, the $z$ component of the angular momentum, mh, is conserved. Since the present study involves $j=0$ initially, m is zero throughout. In all subsequent equations $\mathrm{x}$ is understood to be zero.

Inserting eq. IV-7 into the time-dependent Schrodinger equation results in the coupled Equations

$i h \dot{C}_{v j}(t)=E_{v j}{ }^{o} C_{v j}+E_{v^{\prime} j}, O_{v^{\prime} j^{\prime} v v} C_{v^{\prime} j^{\prime}} \varepsilon_{o} \sin (\omega t+\delta), \quad I V-8$ 
where the $E_{v j}^{\circ}$ are eigenvalues of $\hat{H}_{o}$ and the $D_{v}{ }^{\prime}{ }^{\prime} v j$ are matrix elements

$$
D_{v^{\prime} j^{\prime} v_{j}}=-\int_{0}^{\infty} R_{v^{\prime}} d(r) R_{v} d I \times\left\{\begin{array}{l}
{\left[\frac{(1+1)^{2}}{(2 j+1)(2 j+3)}\right]^{\frac{1}{2},} j^{\prime}=j+1} \\
{\left[\frac{j^{2}}{(2 j-1)(2 j+I)}\right]^{\frac{1}{2},} j^{\prime}=j-1} \\
I V-9
\end{array}\right.
$$

It vill be shown later, as with the classical results, that the laser phase $\delta$ does not appreciably effect the results. For efficiency, the majority of the ruantum calculations are made with a fixed $\delta$ of $\pi / 2$. The coefficients $C_{v j}$ of eq. IV- 8 must be complex. Thus, writing $C_{v j}=X_{v j}=i Y_{v j}$, one obtains the coupled real equations $-n \dot{Y}_{v j}-E_{v j}^{0} X_{v j}+E_{v^{\prime} j} D_{v^{\prime} j^{\prime} v j} X_{v^{\prime} j} E_{0} \sin (\omega t+\delta)$ $h \dot{X}_{v j}=E_{v j}^{0} Y_{v j}+\Sigma_{v^{\prime} j^{\prime}} D_{v^{\prime} j^{\prime} v_{j}} Y_{v^{\prime} j^{\prime}} E_{o} \sin (\omega t+\delta)$.

For comparsion with the classical results, we will also be Incerested in the cransition probahilities

$$
P \underset{v j}{Q M}(t)=\left|c_{v j}(c)\right|^{2} \text {, }
$$

the energy absorption

$$
\langle E(t)\rangle_{Q H}=\varepsilon_{v j} \quad P_{v j}^{Q M}(t) \quad E_{v j}^{\circ}-E_{1} \text {, }
$$

and the pulse length averaged energy absorption, 


$$
\vec{E}_{Q M}(\omega)=\frac{1}{T} \int_{0}^{T}\langle E(t)\rangle_{Q M} d t .
$$

We also study the nonrotating case in a similar fashion. One may obtain the nonrotating equatiors by omitting all factors that include $j$ and $m$ in eqs. IV-7 throu's. IV-I2.

\section{Computational Details}

A Morse potential, $v=D\left(e^{-\alpha\left(r-r_{e}\right)}-1\right)^{2}$, was used, with the parameters corresponding to HF taken from Ref. $52: D=0.22509$, $c=1.1741$, ard $r_{e}=1.7329$ a.u. Since relatively low $v$ states are involved, a linear approximation to the dipole function is satisfactory, $d(r)=d_{0}+d_{1}\left(r-r_{e}\right)$, with $54 c d_{0}=0.716$ and $d_{1}=0.310$ a.u. (1Debye $=0.39343 \mathrm{a.u}$.$) . Some work, in fact, was done with a$ quadratic form for $d(r)^{53 c}$, and that did not significantly affect our resules.

Laser intensities of 1.0 and $2.5 \mathrm{TW} / \mathrm{cm}^{2}$ were used, which correspond to field strengths $E_{0}$ of 0.005338 and 0.008440 a.u. respectively. (IVolt/cm $=1.9447 \times 10^{-10}$ a.u.) The matrix elements $D_{v} j^{\prime} v j$ of eq. IV-9 were evaluated numerically, although analytical forms do exist 58 . Some typical elements are $D_{1100}=0.022, D_{2211}$ $=0.028$ and $D_{2011}=0.032$ a.u.

For the classical rotating HF calculations, 1000 trajectories with random inicial conditions (see Appendix B) were run for most frequencies. Monce-Carlo errors in the quanticies of interest were 
between 10 and $15 \%$. For the nonrotating calculations, 50 trajectories were run for each frequency. In this case it is more efficient to increment the vibrational angle variable in a step-wise fashion between 0 and $2 \pi$ than to pick it randomly. The classical equations of motion were integrated with a standard predictor-corrector routine 59 to either 0.9 or 1.5 ps. The trajectories were back-integrable to four significant figures in all variables. Integration of the classical equations beyond about 1.5 ps is extremely difficult due to the accumulation of error. The integration of oscillatory nonlinear differential equations over long time periods is still a current problem of numerical analysis ${ }^{60}$.

By between 0.9 and 1.5 ps, the pulse averaged energy absorption, eq. IV-6, appears to be converging, but has thot yet fully converged. However, reasonable estimates of the converged $\bar{E}_{\mathrm{CL}}$ can be abtained, since $\langle E(t)\rangle_{\mathrm{CL}}$ has either leveled off to some extent or is oscillating with a small amplitude.

The quantum equations of motion, eqs. IV-10, were integrated with the same predictor-corrector routine as the classical equations. An adequate basis set for HF with the intensities and time scale of interest consisted of the first five $v$ and first five $j$ states, $i$. a 25 term expansion. The nonrotating quantura solutions were obtained In a similar fashion, with five $v$ states being sufficient. Most of the quantum solutions were integrated to 2 ps, although when the laser frequency was near a multiphoton resonance, it was necessary to 
integrate to times in the 10 to 20 ps range in order to obtain converged pulse time averages. Interestingly, because the quantum equations are linear, it is possible to integrate the 50 coupled quantum equations to times exceeding $20 \mathrm{ps,} \mathrm{which} \mathrm{is} \mathrm{much} \mathrm{longer}$ than it is practical to integrate only four nonlinear classical equations.

To aid in the interpretation of the results, Table VIII gives some relevant $\mathrm{E}_{\mathrm{vj}}^{\mathrm{O}}$ levels for $\mathrm{HF}$, calculated with the rotating Morse oscillator formula 57 .

B3. Resul.ts and Discussion

The quantum and classical pulse time averaged energy absorption spectra are plotted in Fig. $12 \mathrm{a}$ for nonrotating and Fig. 12b for rotating HF, with laser intensity $1.0 \mathrm{TW} / \mathrm{cm}^{2}$. The $\mathrm{plot}$ for nonrotating $\mathrm{HF}$ is sinflar to plots of Walker and Preston 52 for higher intensicies. At $1.0 \mathrm{TW} / \mathrm{cm}^{2}$, though, the quantum structure is more resolved. The major features are a narrow two-photon resonance at $\bar{v}=\omega / 2 \pi c$ $=3879 \mathrm{~cm}^{-1}$ (i.e. the $v=0$ to $v=2$ absorption), and a broad onephoton resonance at $\bar{v}=3966 \mathrm{~cm}^{-1}$ (the $v=0$ to $v=1$ absorption). The classical spectrum shows just one very broad peak with a maximum near $3940 \mathrm{~cm}^{-1}$. While the classical spectrum does not have any quantum structure, examination of the classical state distribution does show the presence of a small amount of two-photon absorption, as the frequency is lowered. Details of this will be given later. For rotating HF, the spectra (Fig. 12b) are qualicatively 
simflar to the nonrotating case. There are three peaks in the quantum spectrum : one broad peak near $\bar{v}=4006 \mathrm{~cm}^{-1}$, which corresponds to the $(v, j)=(0,0)$ to $(1,1)$ one-photon resonance, and two narrow peaks near $3937 \mathrm{~cm}^{-1}$ and $3879 \mathrm{~cm}^{-1}$, which correspond to the two-photon resonances $(0,0) \rightarrow(2,2)$ and $(0,0)+(2,0)$, respectively. The classical spectrum has one very broad peak which actually peaks near the $(0,0)+(1,0)$ resonance at $3966 \mathrm{~cm}^{-1}$. Overall, the classfcal solution gives a general idea of the absorption. As in the nonrotating case, the classical result predicts more two-photon absorption for frequencles red-shifted from the onephoton resonance, as will be discussed below.

In Fig. 13, the rotating HF average energy absorption for an tntensity of $2.5 \mathrm{TW} / \mathrm{cm}^{2}$ is shown. Qualitatively, the quantum peaks become broader and overlap more than the $1.0 \mathrm{TW} / \mathrm{cm}^{2}$ case. There appears to be a small power shifting of the resonance peaks toward higher Erequencles (see Ref. $54 \mathrm{c}$ for a discussion of this effect), but this has not been clearly resolved here. Classically, the absorption also broadens relative to $1.0 \mathrm{TW} / \mathrm{cm}^{2}$, and the peak maximum appears to shift to lower frequencles, indicating more multiphoton absorption.

We now turn to look at the rotationally averaged transition probabtlitfes (later, we wll examfne specific $v, j$ probabilitles). Table IX shows the quantum and classical time averaged probabilities at varlous frequencles for rotating and nonrotating $H F$, with laser 
intensity $I=1.0 \mathrm{TW} / \mathrm{cm}^{2}$. Each peak of the quantum solution can be seen to be either a one or a two photon absorption, with both processes observed appreciably only where peaks overlap. At higher intensities the peaks will broaden and overlap more. The classical results do indicate the presence of some two-photon absorption as frequency is decreased. But classically, there is a very gradual change which results in the very broad single peak in the spectrum of Fig. 12, rather than the abrupt changes as in the quantum results.

To show some of the effects of fncreasing intensity, average probabilities for rotating HF at $2.5 \mathrm{TW} / \mathrm{cm}^{2}$ are given in Table $X$. For this larger intensicy, both classically and quantum mechanically the excited states becone more populated.

We now examine the energy absorption and transition probabilities as a function of time. In Fig. 14, a comparison of classical and quantum energy absorption as a function of time is given for nonrotating $\mathrm{HF}$ with $\bar{v}=3966 \mathrm{~cm}^{-1}$ being the laser frequency, which corresponds to the $v=0+1$ resonance. The quantum results show oscillations with a period of about 0.75 ps with no sign of damping out to $1.5 \mathrm{ps}$. At this frequency and intensity $\left(1.0 \mathrm{TW} / \mathrm{cm}^{2}\right)$ the solution is well approximated by a two level system ( $1 . e$. the Rabi model ${ }^{61 a}$ ). In contrast, the classical result oscillates with a frequency of about $0.4 \mathrm{ps}$ and a smalier amplitude. Also, it appears as though the oscillations 
may ie damping.

Fig. 15 shows the classical and quantum time-dependent energy absorption for rotating $\mathrm{HF}$ with $\bar{v}=4006 \mathrm{~cm}^{-1}$, the onephoton $(0,0) \rightarrow(1,1)$ resonance. The results are similar to those in Fig. 14 for nonrotating HF. In this case, though, the classical result appears to level off even faster. The behavior of the quancum solution is agata well approximated by the two level Rabi model ${ }^{6 l a}$. The quantum solution has been followed for up to 20 ps with no clear sign of damping.

The quantum result for the two-photor f onance at $3937 \mathrm{~cm}^{-1}$, the $(0,0)+(2,2)$ resonance, is constderably different (Fig. 16). The complicated nature of the ofscllations may be contrasted with the simple Rabi oscillations of Fig. 15. From Fig. 16 it can be seen tiat the two-photon absorption is a long time process. The corresponding classical result (Fig. 17) also seems to show some aspects of the slower growt:h in absorption, although the solution ts reasonably level. by $0.9 \mathrm{ps}$.

In Figs. 18,19 and 20, plots are shown for some transition probabilities as a function of t:ime, again for $I=1.0 \mathrm{TW} / \mathrm{cm}^{2}$. Here, the classical solution is actually broken up into rotational levels, so that the discrepancy with quantum mechanics can be seen. The results for $\bar{v}=4006 \mathrm{~cm}^{-1}$ are given in Fig. 18. The quantum solutions for $P_{01}$ and $P_{1.0}$ are not snown since they are very small $\left(\leqslant 10^{-2}\right)$. Qualitatively, the probabilities show the same behavior as the energy abscrption, 1.e. the classical 
solutions tend to level off more and the quantim solutions appear periodic. Note that in reality there are high frequency, small amplitude osc1llations that are superimposed on the quantum probab1lities, which have not been clearly resolved, and thus glve rise to some roughness, particularly near peak maxima. The classical probabilities for rotating $H F$ at $\bar{v}=3937 \mathrm{~cm}^{-1}$ are shown in Fig. 19. It can be seen that the $v=2$ state gets significantly populated, but the $v=1$ state is also significantly populated. The quantum probabilities at this frequency are shown in F1g. 20. The resonant probability $\mathrm{P}_{22}(\mathrm{t})$ displays a long perfod which essentially matches that of the erergy absorption in Fig. 16. Another reasonably significant probability is $P_{1 I}$, which is not shown. $P_{12}(c)$ displays higher frequency oscillations that can reach a maximum of about 0.13 . The other two-photon resonance, at $3879 \mathrm{~cm}^{-1}$, is not shown here. Qualitatively, the classical results for this frequency show much less excitation than for 3937 $\mathrm{cm}^{-1}$. There is a small amount of $v=1$ excitation and no $v=2$ excltation. Essentially no rotational excitation is seen in the classical results for this frequency. The quantum results for 3879 $\mathrm{cm}^{-1}$ show somewhat less excitation into the $(1,1)$ state than for $3937 \mathrm{~cm}^{-1}$, and again the resonant probab1ltty, $P_{20}$, displays a long period.

Finally, we turn now to the effect of laser phase. Based on the classical and quantum equations of motion, eqs. IV-3 and IV-8, without additlonal approximations, one would expect the 
solutions to be dependent on the choice of laser phase $\delta$. Without allowing for the detalls of how the field is turned on, a complete study should involve averaging over the laser phase to obtain the most meaningful results ${ }^{62}$.

The laser phase depenuence, however, disappears from the quantum equations in the rotating wave approximation ${ }^{61}$, as has been recently shown ${ }^{63}$. However, for suffictently large field strengths or de-tuning of $w$ from resonance, the rotating wave approximation will breakdown ${ }^{61 c}$. Thus, for example, Moloney and Meath ${ }^{62}$ have shown the laser phase dependence of probabilities as a function of time for a two state model. They found Increasing phase effects for larger field strengths and at multiphoton resonances.

The situation is not quite as clear in the classical analysis. However, if only the relative difference between the laser phase and vibrational phase is Important, then it would be sufficient to average over only the vibrational phase, without averaging over the laser phase, 1.e. the laser phase would not matter. The conditions for this to be true probably include that w be close to resonance.

To assess the effect of laser phase $\delta$ on the present problem, consider first nonrotating HF. For an intensity of $1.0 \mathrm{TW} / \mathrm{cm}^{2}$ and frequencies of $3966 \mathrm{~cm}^{-1}$ and $3879 \mathrm{~cm}^{-1}$, the classical solutions were obtained for fixed $\delta$ of 0 and $\pi / 2$. 500 trajectorles were run 
for each solution to ensure no statistical error. Over the entire 1.5 ps range, the energy absorption as a function of time for the two phases agreed to between 2 and 4 significant figures. The quasiclassical probabilities were also in excellent agreement. Simflarly, the nonrotating quantum results for the same conditions showed very little phase dependence.

We also examfned rotating $\mathrm{HF}$ at $1.0 \mathrm{TW} / \mathrm{cm}^{2}$ for the possibility of phase effects. Within the Monte-Carlo error, no clear phase effect can be distinguished in the classical results. However, slight discrepancies in the time-dependent quantum solutions may be seen, sfuce no statistical error is present. Table XI lists some relevant probabtlitfes and $\langle E(t)\rangle_{Q M}$ for $\delta=0$ and $\pi / 2$, at $\bar{v}=4006 \mathrm{~cm}^{-1}$. Other phases were also examined, but the largest differences were between these two phases. Despite the laser frequency befing almost exactly on resonance, slight differences may be noted, particularly in the probabilities. These differences become larger near peak maxima and can be as much as $4 z$. However, such differences are comparable in amplitude to the high frequency oscillations that are superimposed on the Rab1 osclilations, and do not appreclably effect the overall behavior. Table XII presents similar results for $\bar{v}=3937 \mathrm{~cm}^{-1}$. Although this is a two-photon resonance, the discrepancies due to laser phase are comparable to the $\bar{v}=4006 \mathrm{~cm}^{-1}$ results. Thus, for intenstties $\sim 1.0 \mathrm{TW} / \mathrm{cm}^{2}$, and the present frequency range, the effect of laser phase is small and can be neglected for most 
practical purposes.

B4. Summary

The detailed dynamics of both rotating and nonrotating models for $\mathrm{HF}$ in an intense ?aser field has been investigated with both classical and quantum mechanics.

It is found that classical mechanics does not predict the correct rotational state distributions. Also, the time behavior of the classical solution can be qualitatively different frcm the quantum one. Classical mechanics does give the correct magnitude of pulse averaged quantities, such as the average energy a-sorption, but does not give the detailed resonance peaks for multiphoton absorptions. Classical mechanics does correctly indicate the presence of increased multiphoton absorption as the frequency is red-shifted from the one-photon resonance, but it predicts far too little such absorption.

The laser phase has clearly been shown to be unimportant for the intensities and frequencies studied here, but could conceivably be important for other parameter ranges.

It is difficult to extend these conclusicns to polyatomic systems in intense lasers, which are of greater interest. These results do indicace that some care should be taken when classical mechanics is applied to molecular systems. There is the possibility, however, that the increased number of states in: polyatomic could make classical mechanics a better approximation to quantum mechanics than for the present case of a diacomic. The same may be true for a diatonic initially in anexcited state, or in a more intense field, where more states may become populated. 
C. The Period Advance Map and the Simple Pendulum Model

\section{C1. Preliminaries}

In sec. B it was remarked that classical mechanics is sometimes used in the theoretical description of laser-molecule processes $^{51}$. Although some important cautionary notes were given, particularly with regard to the fine details of the absorption, classical mechanics does describe many $f \in$ cures of the absorption in a qualitatively correct manner. Mc eover, as the number of degrees of freedom is increased, 0 , as the Intensity is raised, it is expected that slassical cechanics would provide a better description.

Given that a classical description of molecular energy absorption is to some extent correct, there is 111 a need to understand more clearly the nature of this escription. Most classical trajectory studies, such as th se described in sec. B, are essentially numerical experiments, involving the solution of many coupled nonlinear differer sal equations. There is a need to understand how and why , solution comes out as it does and, moreover, to build more general models of the process that do not require any detaile numerical calculations. In this section, the simplest mor 1 of a laser induced process, the excitation of a nonrota ng diatomic molecule in an intense laser, is examined class ally in some detafl. First, a new approach to the solution, ba ed on generating a map from 
Initial conditions to the solution after one period of oscillation of the laser, is presented. Repeated applications of this period advance map ${ }^{13}$ then allow the solution over many periods of oscillation to be obtained with relative ease. The behavior of the solution, as a function of time, or the number of oscillations of the laser field, is found to be quite interesting, with spirals or "whorls" developing tn phase space. Such behavior has been discussed by Berry and co-workers ${ }^{64}$ for simpler maps, and can be related to some elementary properties of the period advance map. We then go on to show that a very simple rodel, due primarily to Chirikov ${ }^{14}$, and based on the orbits of a pendulum, is capable of describing chis behavior reasonably well. The pendulum model is found to be a useful tool for understanding other features of the absorption, such as certain trends in the average energy absorption as a function of initial vibrational state of the molecule.

In sec. $C 2$, the peric advance map and its generation are discussed. Sec. C3 applies the method to the problem of a nonrotating $\mathrm{HF}$ molecule in an intense ( $\mathrm{TW} / \mathrm{cm}^{2}$ ) laser fielc. Sec. C4 uses the pendulut model to interpret the results of sec. $C 3$, and sec. C5 discusses energy absorption trends in a qualitative fashion with the pendulum model. Finally, in sec. C6, some brlef concluding remarks are given. 
C2. The Period Advance Map

Consider a one-dimensional nonlinear oscillator, driven by a periodic force $f(x, t)=f(x, t+\tau)$. The Hamilconian is writcen

$$
H(p, x, t)=p^{2} / 2 \mu+V(x)+f(x, t), \quad I V-14
$$

where $p$ and $x$ are the canonically conjugate momentum and position, 1 is the mass, and $V(x)$ is the unperturbed potential function. Hamilton's equations are eriodic in time and read

$$
\begin{aligned}
& \dot{f}(t)=-\partial H / \partial x=\dot{p}(t+\tau) \\
& \dot{x}(t)=\partial H / \partial p=\dot{x}(t+\tau) .
\end{aligned}
$$

In many applications, such as those in Ref. 51, the periodicity of eqs. IV-15 is not used as a practical simplificarion. Racher, a direct numerical integration, for each trajectury in a given ensemble, is performed over many periods $T$ of the driving force. If eqs. IV-15 were linear, however, Eloquet analysis 54,65 does allow one to make effective use of the periodicity. Moreover, in the quantur analogue of the problem, because the differential equations for the coeffici nts of the vavelunction are always linear, even when the corresponding classical equations are nonlinear, the periodicity again simplifies the calculations 52,54 . It was the apparent lack of a piactical scheme, making use of the periodicjty of the nonlinear classical equations, that was the 
original motivation for this we to.

The approach taken here is based on the period advance map ${ }^{13}$, which maps initial conditions $\left(p_{0}, x_{0}\right)$ to the corresponding solution at $t=\tau$, $\left(P_{T}, x_{T}\right):$

$$
\left(p_{\tau}, x_{\tau}\right)=T\left(p_{0}, x_{0}\right)
$$

An elementary property of the periodicity of eqs. IV-15 is that once the solution over the first period is known, then it is known for all subsequent period advances by repeatedly applying the map :

$$
\left(p_{r \tau}, x_{r T}\right)=T^{r}\left(p_{0}, x_{0}\right), r=1,2,3, \ldots
$$

In addition to the useful and practical property IV-17, it is a straightforward consequence of Liouville's theorem that $T$ is an area preserving map ${ }^{13}$.

A functional representation of eq. IV-16 is

$$
\begin{aligned}
& T_{p}\left(p_{o}, x_{0}\right)=P_{\tau} \\
& T_{x}\left(p_{0}, x_{0}\right)=x_{\tau}
\end{aligned}
$$

where $T_{p}$ and $T_{x}$ are two-dimenstonal functions spanning the relevant phase space. To obtain approximations to $T_{p}$ and $T_{x}$, one may simply define a grid of initial conditions $\left(p_{0}^{i}, x_{0}^{j}\right)$ and for each grid point $(i, j)$ 
numerically integrate over one perlod of the motion to obtain $T_{p}^{1 f}$ and $\mathrm{T}_{\mathrm{x}}^{1 \mathrm{j}}$. Any appropriate two-dimensional fitting function may be used to define the approximations to $T_{p}$ and $T_{x}$. of course to obtain accurate approximations, a dense grid of tnitial points, such as a $100 \times 100$ grid, must be ined. However, the numerical integration of even a very dense grid over only one period of the driving force is usually a very quick and easy task. Furthermore, once $T$ has been satisfactorily determined, the solution over many periods of oscillation is known from eq. IV -17 .

In the calculations reported below, use was made of two-dimenstonal cubic spline functions to define $T_{p}$ and $T_{x}$. The advantage of using splines is that they pass uniquely through all the data points, and can be shown to have desirable minimum curvacure properties ${ }^{66}$. A cubic spline is a piecewise continuous interpolating polyanomial with continuous first and second derivatives. A possible disadvantage of using splines is then that higher order derivatives may not be continuous. However, no problems were encountered in che present application. The explicit algorithm used here is that of Ref. $66 \mathrm{~b}$, with the one-dimensional splines required by this algorithm chosen to be the natural cubic splines of Ref. 66 a. 
c3. Application to a Diatomic in an Intense Laser

\section{Action-Angle Variables}

Frequent use will be made of the Morse oscillator action-angle variables, so it is convenient to define them here. The Hamiltonian for a Morse osclllator, in the absence of any driving force, is

$$
H_{c}(p, x)=p^{2} / 2 u+D\left(e^{-\alpha x}-1\right)^{2} \text {. }
$$

where $D$ is the dissociation energy, and $\alpha$ is a positive constant related to the curvature of the potential. For convenience, $x$ has been taken to be the displacement from equilibrium. It is possible to change variables frow $(p, x)$ to action-angle variables $(n, q)$ such that $H_{0}=H_{0}(\Omega)$, i.e. the new Hamiltonian is a function of the action $n$ only. Rankin and Miler ${ }^{67}$ have done this for the Morse oscillator, with the result

$$
H_{0}(n)=(n+h / 2) \omega_{0}-(n+n / 2)^{2} w_{0}^{2} / 4 D
$$

where $\omega_{0}=\sqrt{2 \mathrm{Da} / \mu}$. The unperturbed motion is then given by $\dot{\mathrm{x}}=0$, and $\dot{q}=\omega(n)$, where the oscillator frequency is

$$
\omega(n)=\partial H_{0} / \partial n=\omega_{0}-(n+h / 2) \omega_{0}^{2} / 2 D
$$

The old variables $(p, x)$ are related to the new variables $(n, q) b y$ 


$$
\left.\begin{array}{rl}
x(n, q) & =\alpha^{-1} \ln \left[\left(D+\sqrt{\mathrm{DH}_{0}(n)} \cos q\right) /\left(D-H_{0}(n)\right)\right] \\
p(n, q) & =\mu \omega(n) \partial x / \partial q \\
& \left.=-\mu \alpha^{-1}\left[\omega_{0}-\vdots n+\pi / 2\right) \omega_{0}^{2} / 2 D\right] \frac{\sqrt{D_{0}} \operatorname{sinq}}{\left(D+\sqrt{D H_{0}} \cos q\right)}
\end{array}\right\} I V-22
$$

The phase convention in eqs. IV-22 has been chosen so that $q=0$ is an outer turning point and $q=\pi$ is an inner turning point.

It should be noted that $n$ corresponds closely with the vibrational quantum number $v$. Thus if one replaces $n$ with $v, v=0,1, \ldots$ eq. IV-20 gives the correct energy eigenvalue formula for the Morse oscillator. We use atomic units $(h=1)$ throughout, so that classically $n$ can take on any value $\geqslant-1 / 2$ and $0 \leqslant q \leqslant 2 \pi$.

Generating the Period Advance Map

To mimic a nonrotating $\mathrm{HF}$ molecule in a strong laser, a Morse potential was used with a driving force linear in $x$ (i.e. the linear dipole approximation),

$$
H(p, x, t)=p^{2} / 2 \mu+D\left(e^{-\alpha x}-1\right)^{2}-\varepsilon x \cos 3 t
$$

with $E=\sqrt{8 \pi \mathrm{I} / \mathrm{c}} \mathrm{d}_{1}$, where $\mathrm{I}$ is the laser intensity, $c$ the speed of light, and $d_{1}$ the slope of the dipole. For HF, as before, we have ${ }^{52} D=0.22509$, $\alpha=1.1741$ and $\mu=1744.8 \mathrm{a} . u$. With $\mathrm{d}_{1}=0.3099 \mathrm{a.u.}, \varepsilon=0.00165 \mathrm{a.u}$. for a $1 \mathrm{TW} / \mathrm{cm}^{2}$ laser intensity. The laser frequency was taken to be the fundamental transiton frequency, i.e. $\Omega=H_{0}(1)-H_{0}(0)=0.01807$ a.u. The period is then $\tau=2 \pi / \Omega=347.7 \mathrm{a} . \mathrm{u}$. (or $0.00841 \mathrm{ps}$ ). 
The period advance map was then obtained by the method described in sec. C2. A suitable grid for the present purposes was a $100 \times 100$ grid with $x_{0}$ and $p_{0}$ evenly spaced between $-0.3573 \leq x_{0} \leq 0.6274$ and $-14.61=\mathrm{P}_{0}=14.61$. These liwiting values allow for excitations up to $\mathrm{n}=3$. As a check on the accuracy of the map so obtained, comparsion was made with trajectories that were directly integrated over 5 periods of the driving force. In Table XIII is shown the root mean square deviations of the predicted $p_{\tau \tau}, x_{\tau \tau}$ values from the map and the directly integrated trajectories for an ensemble of 50 trajectries with $n=0$ initially and $q$ evenly spaced between 0 and $2 \pi$. The map is seen to be accurate over the first few hundred periods. However, the numerical interpolation error builds up, particularly in $P_{I \tau}$, as I increases, and the map cannot be expected to be very accurate beyond $r-400$. This ertor could be improved, of course, if a finer grid, particularly in $p_{0}$, were used, but the present form is sufficient for our purposes.

It should be noted that although it was necessary to integrate numerically 10,000 crajectories over one period of the driving force, the resulting map contains information chat would be much more difficult to obtain by direct integrations over many periods of oscillation. For example, the behavior of che entire phase plane will be examined below with the map. 
Repeated Iterations of the Map

If one takes some initial set of $(p, x)$ points and repeatedly maps chem, plotting each mapped point, one obtains the surface of section plot shown in Fig. 2la. Points on each curve labelts A. :hrough E will, upon successive mappings (or perio.s of oscilintori), yield points on the same curve. Thus, for example, if one stets tr some point on curve $C$, it will, upon repeated mappings, travel counterclockwise around $C$ in uneven but discrete jump The $F$ itot $T$ fixed points, $i . e$. those points satisfying $(p, x)=T(p, x)$, lif $b y$ symmetry of the underlying Hamiltonian, n the $x$ axis. - ise n are classified as being stable elliptic (o in Fig. 2I) :r unst' $e$ hyperbolic(•) fixed points depending on whether mapped points, ir small region about each fixed point, fall on closed el. ' ses or upen hyperbolas. The ell1ptic or hyperbolic nature of each fixed polll was also verified by a Linearizacion of the map about ea fixer, at With $T$ defined in terms of the cubic splines, the requiret ferivat could be obtained analytically.

As a check on the accuracy of the map, the fixed points wer: als. found by direct integrations of trajectories and agre: : to six significant figures, with the map predictions. Incitentally, there is one more fixed point, that lies at $x-\infty$, so that the tol..1 number of Iixed points is even, as would be expected 68 . There. $=350,1 \mathrm{gl}$ orjer fixed points, $\langle p, \cdot\rangle=T^{s}(p, x), s>1$, wh. $1,1: 1$, mentioned later on. 
Also, the approximate positions of the period 1 flxed points can be predicted remarkably well with some simple arguments given in Appendix $C$.

It may appear, from Fig. 21a, that the motion is quite complicated. However, if one plots the curves in action-angle space, with $n$ and $q$ as defined previously, one obtains Fig. 21b. The curves in $(n, q)$ space bear a close resemblance to orbits of a simple pendulum ${ }^{14,69}$. In fact, this analogy will be made mora clear in sec. C4. By symmetry, the three relevant fixed points now lie at either $q=0$ or $q=\pi$. The two most important of these points are $(n, q)=(0.61726, \pi)$, which is stable, and $(0.36030,0$, which is Lastable. Notice that these points have action values near $n=1 / 2$, which is the action where the molecular frequency is in resonance with the laser frequency $(\omega(1 / 2)=\Omega)$. The other fixed po: .t, which lies at $(-0.48206,0)$, and is stable, corresponds to the molecule having practically no energy. Some orbits, very near thls point, wust close around $1 t$. However, this occurs only In a very sma? region and most orbits, even for $n$ values as ;mall as -0.4, appear as "rotations" (e.g. curve E 1n F1g. 21b). We will not concern ourselves with the region very close to this point.

As a final point on the surface of section plots, no "chaotic" $\because$ gton were noticable with the present perturbation strength $\varepsilon$. of course these regions do exist, particularly near the separatrix curves $B$ and $D$, which are the sets of points which eminate from and approach the unstable fixed point, tut are small in area ard difficult to see on the gcale of the plots. 
It is of interest to see how a line in action-angle space, which corresponds to an inttial classical vibrational state, behaves under the mapping. In Fig. 22 is shown the result of mapping points that lie Inttially on a line in $(n, q)$ space with $n_{0}=1 / 2$ and $q_{0}$ between 0 and $2 \pi$. It is seen that the line distorts into quite a remarkable structure. Spirals or whorls develop near the sitable fixed point (o). Although not shown here, even more applications of the map results in the whorls winding more and more about the stable flxed point. The behavior near the unstable flxed point (•) is also interesting. Initially no point lies on the unstable fixed point, but very quickly under the mapping points begin to approach it closely. Fig. 23 shows the results of mapping points that lie initially on Iines with $n_{0}=0$ and 1 . The behavior of these initial states is similar to $n_{0}=1 / 2$, with, for example, whorls again evolving about the stable fixed point.

Berry and co-workers ${ }^{64}$ have considered the fate of curves under mapplngs. They expect to see whorls near stable fixed points, which Indeed are observed here. In the next section, this behavior w111 be seen to also arise out of the pendulum analogy ${ }^{14}$. Berry an + coworkers ${ }^{64}$ also predict "tendrils", or chaotic and snake-1ike convolutions, to develop near the unstable fixed point. Tendrils are not observed here. The reason that the tendrils are hard to see is that they are associated with the chaotic motion near the separatrix. As we have already noted, this region is quite small here, so that tendrils are not evident on the scale of Figs, 22 and 23 . However, 
if one expanded the scale, and looked very carefully, tendrils may be evident.

Another interesting manffestation of the whorls is evident in a coarse-gralned glance at the entire phase plane evolving under the map. Constder dividing the inftial phase space into, say, a $120 \times 120$ grid of points with $-1 / 2 \leq n_{0} \leq 5 / 2$, and $0 \leq q \leq 2 ;$. Each point of this grid is then mapped and the resulting new action at time $r \tau, n_{r \tau}\left(n_{0}, q_{0}\right)$, is plotted in a coarse-grained fashion. If $n_{r \tau}$ is between $-1 / 2$ and $1 / 2$, corresponding to a crude $\mathrm{a}=0$ state, white coloring is used. If $\mathrm{n}_{\mathrm{rt}}$ is between $1 / 2$ and $3 / 2$, corresponding to an $n=I$ state, grey coloring is used. If $\mathrm{n}_{\mathrm{rt}}$ is between $3 / 2$ and $5 / 2$, an $\mathrm{n}=2$ state, diagonal Iines are used. Finally, if $\mathrm{n}_{\mathrm{rt}}$ is between $5 / 2$ and $7 / 2$, an $\mathrm{n}=3$ state, dots are used. At time zero ( $1 . e . r=0)$, one has simply rectangular blocks wh white on bottorr, grey on top of the white, and diagonal lines on top of the grey. After 20 periods of oscillation of the laser, one obtains F1g. 24a, which shows a developing whorl in the $n=1 / 2$ resonance region. Notice that the axes in Fig. 24 are always the Initial action and angle variables, and the coloring represents the coarsu-grained action at time $r T$ that evolved from those initial conditions. After 40 and 60 oscillations one obtains Figs. $24 \mathrm{~b}$ and $24 \mathrm{c}$, with the whorl winding more tightly in the resonance region. Although not shown here, this has been followed for up to 400 perlods of the driving force, with the whorls winding tighter and tighter, representing very graphically how molecules absorb and emit 
energy in the vicinity of a resonance.

C4. The Pendulum Model

The remarkable similarity of the surface of section plot Fig. $21 \mathrm{~b}$ and the pendulum phase plane orbits ${ }^{14,69}$ can be mate more concrete. In fact, Chirikov ${ }^{14}$ has developed a theory of classical resonance based on this analogy. Here, similar Ideas are applied to the forced Morse oscillator problem of sec. C3.

Derivation of the Pendulum Hamiltonian

In action-angle variables, the full Hamiltonian is

$$
H(n, q, t)=H_{0}(n)-E x(n, q) \cos \Omega t . \quad I V-24
$$

In our problem, $x(n, q)$, defined by eq. IV-22, is an even function of $q$ so that the perturbation may be expanded in a cosine Fourier series with the result

$$
x(n, q) \cos \Omega t=\sum_{k=0}^{\infty} v_{k}(n)[\cos (k q-\Omega t)+\cos (k q+\Omega t)],
$$

where

$$
V_{k}(n)=(2 \pi)^{-1} \int_{-\pi}^{\pi} d q x(n, q) \cos k q . \quad I V-26 a
$$


The integral of eq. IV-2'oa may be evaluated analytically ${ }^{70}$ for $x(n, q)$ given by eq. IV-22, to yield explicit expressions for the Fourfer coefficients,

$$
\begin{aligned}
& v_{k}(n)=-d^{-k} / \alpha k, k \neq 0 \\
& v_{0}(n)=a^{-1} \ln \left(-\sqrt{H_{0} / D} d / 2\right),
\end{aligned}
$$

where $d=-\sqrt{D / H_{0}}\left(1+\sqrt{\mathrm{I}-\mathrm{H}_{0} / D}\right)$.

Now the important terms in the expansion of eq. IV-25 are those with slowly varying arguments. If one is looking at a region of phase space with the action $n$ near $n_{1}$, the primary resonance defined by

$$
\omega\left(n_{1}\right)-\Omega=0
$$

where $w(n)$ is the osclllator frequency given by eq. IV-21, then the term $q-\Omega t$ is slowly varying, since $\dot{q}-\Omega=\omega\left(\Omega_{1}\right)-\Omega=0$. Thus one may keep only the relevant $\langle k=1$ is eq. IV-25) term in the Fourier expansion, which results in the approximate Hamiltonian, valid near $n=a_{1}$, of

$$
H(n, q, t) \approx H_{0}(n)-\varepsilon V_{1}(n) \cos (q-\Omega t),
$$


where the $\cos (q+\Omega \tau)$ term has also been assumed very osctllatory and neglected. Next, one transforms to a new angle vartable $\hat{q}=q-\Omega t$, which corresponcs to the difference between molecular and laser phases. This may be accomplished with a generator ${ }^{14,69} F_{3}(n, \hat{q}, c)=-\left(n-n_{1}\right)(\hat{q}+\Omega t)$, which implicitly defines the new variables $\hat{p}$ and $\hat{q}$ through $\hat{n}=-\partial F_{3} / \partial \hat{q}$ and $q=-\partial F_{3} / \partial n$. This results in $\hat{p}=n-n_{1}, \hat{q}=q-\Omega t$, and the new Hamilconian

$$
\begin{aligned}
K & =H(\hat{p}, \dot{q}, t)+\partial F_{3} / \partial t \\
& =H_{a}\left(\hat{p}+n_{1}\right)-\varepsilon V_{1}\left(\hat{p}+n_{1}\right) \cos \hat{q}-\hat{p} \Omega .
\end{aligned}
$$

The final step is to expand $H_{0}$ about $\hat{j}=0$ (1.e. $n=n_{1}$ ) to quadratic order and $v_{1}$ to zero order. Since $H_{0}$ is already quadratic in $n$ (see eq. IV-20), che expansion of $H_{0}$ is exact here. The result is

$$
K-H_{0}\left(n_{1}\right)=\hat{p}^{2} / 2 M_{1}-\epsilon V_{1}\left(n_{1}\right) \cos \hat{q} .
$$

where $M_{1}^{-1}=(\partial \omega / \partial n)_{n_{1}}$. Eq. IV-30 is the Hamiltonian for a pendulum of mass $M_{1}$, which for the Morse oscillator, from eq. IV-21, is

$$
M_{1}=-2 D / \omega_{0}^{2} \leq 0
$$

To make the connection with the more famtlar posttive mass pendulum orbits, one may note that the equations of motion consistent with eq. IV-30, 


$$
\begin{aligned}
& \dot{\hat{p}}=-\partial K / \partial \dot{q}=-\varepsilon V_{1}\left(n_{1}\right) \sin \hat{q} \\
& \dot{\hat{q}}=\partial K / \partial \hat{p}=\hat{p} / M_{1}
\end{aligned}
$$

are the same as

$$
\begin{aligned}
& \dot{\hat{p}}=E V_{1}\left(n_{1}\right) \sin \hat{\theta} \\
& \dot{\hat{\theta}}=\hat{p} / m_{1}
\end{aligned}
$$

with $\hat{\theta}=-\hat{q}$ and $m_{1}=-M_{1}>0$. But eqs. IV-31 are consistent with the Hamilonian

$$
K^{\prime}=\hat{p}^{2} / 2 m_{1}+\varepsilon v_{1}\left(n_{1}\right) \cos \hat{\theta}
$$

where $(\hat{p}, \hat{\theta})$ are the confugate variables. Thus the well known pendulum orbits ${ }^{14} \hat{p}(t), \hat{\theta}(t)$ will determine the approximate motion of a forced Morse oscillator when the action is close to $n_{1}$ through $n(t)=\hat{p}(t)+n_{1}$, and $q(t)=\delta(t-\hat{\theta}(t)$.

The separatrix associated with eq. IV- 32 wi]l have waximum and minimum values of action for $\hat{\theta}=\pi$ and $K^{\prime}=\varepsilon V_{1}$, if $V_{1}>0$. If $V_{1}<0$, then they occur for $\hat{\theta}=0$. In either case, the maximum and minimum separatrix actions may be obtained, in terms of the original Morse action variable, from

$$
n_{1}^{ \pm}=n_{1} \pm 2 / \varepsilon \bar{\varepsilon} \overline{v_{1} / m_{1}} .
$$


A similar analysj.s may be made about other resonances. For example, the next resonance corresponds to an "overtone" resonance, $2 \omega\left(n_{2}\right)-\Omega=0$. In this region of action space, $2 q-\Omega c$ is slowly varying so that the $k=2$ Fourier term in eq. IV-25 may he singled out. The same analysis as above, but with a classical genarator of the form $F_{3}(n, \hat{q}, t)=-\left(n-n_{2}\right)(\hat{q}+\Omega t) / 2$, yields

$$
K_{2}^{\prime}=\hat{p}^{2} / 2 m_{2}+\varepsilon V_{2}\left(n_{2}\right) \cos \hat{\theta} .
$$

where now $\hat{p}=\left(n-n_{2}\right) / 2, \hat{\theta}=\Omega c-2 q$, and $m_{2}^{-1}=4 / \partial \omega /\left.\partial n\right|_{n_{2}}$. The fixed points associated with the $n_{2}$ resonance are actually period 2 fixed points of the period advance map. The maximum and minimum separatrix action values, in the original Morse action, are

$$
n_{2}^{ \pm}=n_{2} \pm 4 \sqrt{\varepsilon\left|v_{2}\right| m_{2}} \text { [v-j4 }
$$

In orciar for the pendulum model to be valid, the neglected terms in the Fourier expansion must Indeed be rapidly oscillating compared to the cerms key. Chirikov't has shown that a condition for this to be is the condition of moderate nonlineartty,

$$
E \because \alpha_{r}=|\partial \omega / \partial n| \omega / n \ll 1 / E .
$$

It is also necessary that the separatices associated with, say, the primary resonance $n_{1}$ and the next resonance $n_{2}$, do not overlap much - 
1.e. are well separated from each other. Chirikov ${ }^{14}$ defines the coupling constant of the resonances, $s$, such that if

$$
s=\Delta w / \Delta \therefore 1 \text {, }
$$

then the relevant resonances are sufficiently separated from one another. In eq. IV-36, sw could be the frequency full width of the separatrix of the primary resonance, $\Delta \omega=|\partial \omega / \partial n|\left(n_{1}^{+}-n_{1}^{-}\right)=4 \sqrt{\varepsilon\left|v_{1}\right| / m_{1}}$, and $\Delta$ the difference between the primary resonance frequency $\omega\left(n_{1}\right)$ and the next resonance frequency $\omega\left(n_{2}\right)$. If $s \neq 1$, then the overlap of resonances is associated with chaotic behavior in the vicinit: of the separatrices ${ }^{14}$.

Pendulum Approximation to Nonrotating HF in a Strong Laser

For the problem of sec. $c 3$, it is readily found that $n_{1}=1 / 2$, $m_{1}=1266$, and $\varepsilon V_{1}\left(n_{1}\right)=2.04 \times 10^{-4}$, using the formulae given above. The moderate nonlinearicy condition, eq. IV-35, is also found to be sacisfled, since then $\varepsilon \sim 0.002$, and near $n_{l}=1 / 2, \alpha_{r} \sim 0.02$. The resonance coupling constant $s$ also satisfles eq. IV-36, since the next resonance is $n_{2}=12$, and is thus well separated from $n_{1}\left(s \leq 10^{-1}\right)$.

From the above considerations, one expects that the pendulum model w1ll be reasonably adequate. Indeed, if one uses eq. IV-33 to estimate the maximum and minimum separatrix action values, one finds $\mathrm{n}_{1}^{+}=1.5$ and $\mathrm{n}_{\mathbf{1}}^{-}=-0.5$, in reasonable agreement with the actual values, which from Fig. 21 b are closer to 1.6 and -0.35 . 
The fixed points of the pendulum descrfbed by eq. IV-32, when converted back to the orfginal action-angle variables, nccur at $(n, q)=(1 / 2, \pi)$ and $(1 / 2,0)$, and are stable and unstab respectively. These rixed points agree reasonably well with the two most important fixed points of the problem discussed in sec. C3. The i ndulum model does not have the additional ffxed point, near $n=-1 / 2$, that accurs In the full problem. However, the immdelate region near thy point is of no concern to us here, and, furthermore, the pendulum , bits do actually describe the motion near (but not very near) this ofnt reasonably well, as might be guessed from inspection of Fig. $21 \mathrm{~b}$.

The pendulum equations of motion, eqs. IV-31, were solved numerfcally, since although analytical solutions do exist, in terms of elliptic integrals ${ }^{14,65}$, they are somewhat cubersome to use. Fig. 25 shows the result of following the behavior of classtcal states with $n_{0}=1 / 2,0$ and 1 , just as was done $f n$ sec. C3. By comparison with Figs. 22-23 for the full problem, it is seen that the major qualitative features of the dynamics, including the whorls, arise out of the pendulum equations of motion. It will also be noticed from Fig. 25 that there is an artifact in the pendulum solution in that the action is now no longer bounded from below. Thus some points actually map to below $n=-1 / 2$, which is nonphysical since $n$ for the Morse oscillator is only defined for $n \geqslant-1 / 2$.

Tendrils are absent entirely, even under very close scrutiny, because no chaotic trajectortes exist in the pendulum phase space. The fact tha: they are also difficult to see in the full problem is 
related to the smallness of the resonance coupling parameter s.

It ts also easy to see why points, initfally not on or extremely close to the unstable fixed point will eventually approach it closely. If one starts with an inftial line in $(n, q)$ space, which is also a line in the pendulum phase space $(\hat{p}, \hat{\theta})$, then as long as $\hat{p}=n-n_{1}$ is within the width of the separatrix, the 1ine must Intersect the separatrix twice. Pofnts on the separatrix will tend to move towards an unstable flxed pofnt, or, equivalently, away from another unstable flxed point (cf. curves $B$ and $D$ in Ffg. 21b), but never actually reach the unstable fixed point in finite time. Thus, since all the values of $n_{0}$ studfed are within the separatrix width, necessarily points intersect the separatrix and then approach the unstable fixed point.

The origin of the whorls can be seen in terms of the nonlinearity of the pendulum equations of motion. Because each orbit about the stable fixed point has a dfferent frequency, or rotation number ${ }^{64,68}$, a line that intersects these orbits will then wind about the fixed point, with different points winding faster than other points, thus forming a whorl.

C5. Energy Absorption Trends

In this section, the pendulum model of sec. $C 4$ is used to predict qualitative trends in the average energy absorption $53 \mathrm{c}$ of diatomic molecules initially in different vibrational states. Suppose, for 
example, one has an initlal classical state with $n_{0}$ fixed and $q_{0}$ evenly distributed between 0 and $2 \pi$. This corresponds to a horrfzontal line a-zoss FIg. 2lb. Now each point on the line will evolve, followiug the pendulum-like surface of section lines in the figure. Rather than follow this motion explictly, either by the period advance map, or by direct integration of trajectories, one can use the pendulum model to predict in advance whether there will be a net galn or loss of energy. Suppose $n_{0}$ lies between the minimum separatrix action $n_{1}^{-}$ and the primary resonance action $n_{1}$. Then a substantial number of puints on the Initial line will follow elliptic closed orbits about $n_{1}$. But these points then spend most of their time above $n_{0}$, since $n_{0}<n_{1}$, which results in a net gain of energy (recall $n$ is a measure of the energy - eq.IV-20). Conversely, if $n_{0}$ lies between $n_{1}$ and $n_{1}^{+}$, the maximum in the primary separatrix action, then agaln the points will orbit about $n_{1}$, but spend most of their time below $n_{0}$, resulting in a net energy loss. If $n_{0}$ is outside the separatrices, then the points will simply rotate up and down (as in curve A of Fig. 21b), and, other things being equal, will not gain ir lose much energy. However, if $\mathrm{n}_{\mathrm{o}}$ is increased to within the separat-ix corresponding to the next resonance, $1 . e$. the overtone resonance $n$, there will again be a net energy gain if $n_{0}$ is between $n_{2}^{-}$and $n_{2}$, nd a net energy loss if $n_{0}$ is between $\mathrm{n}_{2}$ and $\mathrm{n}_{2}^{+}$.

Recently, Christoffel and Bowman ${ }^{53 c}$ have looked at the net energy absorption of nonrotating HF molecules as a function of inftial 
vibrational action using classical trajectories. They used a larger perturbation strength than that of secs. C3 and $C 4, E \approx 0.011$, and a slightly different laser frequency, $\Omega=0.01787$ a.u. The pritary resonance is found Erom eq. IV -27 to be $\mathrm{n}_{1}=0.752$. From eq. IV-33, the minimum and maximum separatrix actions are $n_{1}^{-}=-2.0$ and $n_{1}^{+}=3.5$. An action of -2 is nonphysical, but is irrelevant for the arguments that follow. Similarly, the overtone resonance is found from $2 \omega\left(n_{,}\right)-\Omega=0$, and is $n_{2}=12.06$. From eq. IV-34 the associated action extrema are $n_{2}^{-}=9.1$ and $n_{2}^{+}=15.0$. Rounding off crudely, one would expect Initial actions $n_{0}$ between 0 and 1 to have a net energy gain, 1 and 4 to have a net energy loss, 4 and 9 to neither gain nor lose much energy, and once again increased absorption for $n_{0}$ between 9 and 12 . These expectations are in surprisingly good accord with the observations of Christoffel and Bowman 53c. In particular, one could note the increased absorption for $\mathrm{n}_{\mathrm{o}}: 9$. The fact that a great deal of energy, much more than for the lower $\mathrm{n}_{\mathrm{o}}$ values, appears to be absorbed for $n_{0} \geq 9$ is indicative of the higher order resonances $\left(n_{2}, n_{3}, \ldots\right)$ being more closely spaced and overlapping to a large extent, allowing the molecule to essentially travel up a "chaotic ladder" of separatrix layers.

This sort of analysis is entirely qualitative, but does have the advantage that an essentially "back of the envelope" calculation yields qualitatively correct predictions of energy absorption trends. Recently, Davis and Wyatt ${ }^{71}$ have developed a Polncaré surface of section 
approach, based on the numerical integration of only a few classical trajectories, that is complementary to the approach here.

\section{C6. Concluding Remarks}

A method of generating period advance maps ${ }^{13}$ for one-dimensional forced oscillator problems has been given. An application to a forced Morse oscillator demonstrated that a good deal of dynamical information could be obtained from the approach. The applicability is limited, however, to a relatively modest number $(\leqslant 400)$ of map iterations (oscillations of the field), but this can be improved somewhat by increasing the initial grid size. Also, if one is only inceresced in particular dynamical features, such as the face of a specific initial classical state, it is of course easier to use direct classical trajectories.

The behavior of the nonrotating HF molecule in a strong laser: was found to be quite interesting, with classical states developing whorls 64 in the vicinity of the stable fixed point of the map. In fact, the entire phase plane was seen to consist of whorls of excitacion and de-excitation. The pendulum model ${ }^{14}$ of nonlinear resonance seems quite applicable to froblems of this sort and does reproduce some of the major qualitarive features. Also, certain dynamical trends, such as the average energy absorption as a function of initial vibrational state, caln be qualitatively discussed with this model. Since the pendulum model has been used ${ }^{14,72}$ to treat systems with more than 1 or 2 degrees of freerom, there may be some hope in using similar ideas to interpret che behavior of, say, vibrating and rotating diatomic 
molecules in lasers, or more general polyatomics, or perhaps two or more lasers 53 inceracting with a molecule. Indeed, in relaced work, Oxtoby and Rice ${ }^{72}$ have treated the autonomous problem of energy re-distribution in polyatomic molecules after excitation using the pendulum model and Chirikov's resonance overlap ideas for the onset of chaotic motion.

As a final point, the quantum analogue of the classical whor is has not been discussed here. Berry and co-workers ${ }^{64}$ have discussed this point, and do expect to see certain evidence of whorls in the behavior of the time-dependent probability density. 


\section{Concluding Remarks}

Classical and semiclassical methods have been used to treat a variety of phenomena in chemical dynamics.

Perhaps one of the important advantages of such approaches is that one can study reasonably cumplicated problems of experimental interest without too much difficulcy. Thus in sec. II it was possible to make some connection with experiment in the discussion of tunneling in unimolecular reactions. Unfortunately at present there are no experimental or exact quantum mechanical determinations of the unimolecular rate constants for the reactions studied in sec. II, so that it is somewhat difficult to calibrate precisely the semiclassical tunneling models developed. At least the results were compatible with the experimental results that were available.

In sec. III the quite different problem of charge transfer was treated with classical trajectories and a perturbative semiclassical model. In the simple alkali-halogen system studied, accurate quantum mechanical results were available for comparison. It was seen that the classical trajectory results predicted reasonably accurate cross sections over a wide range of energies, suggesting it may be useful to try such approaches with more complicated charge transfer reactions. The semiclassical model was also seen to be useful in the limit of high energy. It is interesting that such seemingly quantum mechanical events, such as nonadiabatic charge transfer, can sometimes be adequately explained within a classical or semiclassical framework. 
In sec. IV, classical methods were used to study a diatomic molecule interacting with a strong laser. Quantum calculations were also made for comparison. It was found that the classical picture was reasorable for predicting certain averaged results, but could not be relied upon for details such as rotational structure in the absorption spectrum. A more detailed classical analysis of a nonrotating diatomic in a laser, based on a novel method of obtaining the classical solution, was sade. A simple pendulum model was used to describe the classical solution, that also proved useful for explaining certain trends in molecular energy absorption.

In conclusion, one must admit that there is some, but not all truth in classical and semiclassical pictures of molecular phencmena, and this line of research is still an interescing and open field for future work (Wyatt's rule ${ }^{73}$ still holds: $d K / d W \gg l$, where $K(W)$ is the knowledge gained as a function of the work exerted).

Jack: Gwendolen, it is a cerrible thing for a man to find out suddenly that all his life he has been speaking the truch. Can you forgive me?

Gwendolen: I can. For I feel that you are sure to change.

from Oscar Wilde's "The Importance of Being Earnest" 
Appendix A. The Reaction Path

The concept of a reaction path is ubiquitous in chemistry, but is often ill-understood and poorly defined. For example, in the past it was often thought to be the path of minimum energy from the transition state down to reactant and product geometries. However, it was later poinced out that such a path is non-unique and discontinuuus 74 .

A more fruitful definition, that does not suffer from the above mathematical faux pas, is the path defined by following the gradient of the Born-Oppenheimer potential 75 . In the following, a very heuristic argument is presented, based on the concept of a zero kinetic energy trajectory connecting reactant, transition state and product geometries, that establishes the differential equations to be satisfied for this path.

Consider the usual (see sec. IIC) mass-weighted Cartesian coordinates, $x_{i y}$. Let $x_{i y}$ be a function of time and Taylor expand it about $t=0$, which is assumed to correspond to a point on the reaction path. We have

$$
x_{i Y}(t)-x_{i Y}(0)=\partial x_{i Y} /\left.\partial t\right|_{O} t+(1 / 2) \partial^{2} x_{i Y} /\left.\partial t^{2}\right|_{0} t^{2}
$$

to second order. But we require that the kinetic energy be zero, so that the velocity $\partial x_{i Y} / \partial t=0$. Also, by Newcon's second law,

$$
\partial^{2} x_{i Y} / \partial t^{2}=-\partial v / \partial x_{i Y},
$$


where $V$ is the Born-Oppenheimer potential surface. Thus

$$
x_{1 \gamma}(t)-x_{1 \gamma}(0):-(1 / 2) \partial V /\left.\partial x_{1 \gamma}\right|_{0} t^{2}
$$

But the distance traveled along the reaction fath is, for small steps,

$$
\begin{aligned}
\Delta s & =\sqrt{\sum_{i \gamma}\left[x_{i \gamma}(t)-x_{i \gamma}(0)\right]^{2}} \\
& =(1 / 2) t^{2} \sqrt{\varepsilon_{i \gamma}\left(\partial v / \partial x_{i \gamma}\right)^{2}}
\end{aligned}
$$

Now divide eq. A2 by eq. A3 and take the limit of infinttesimal $\Delta s$ :

$$
\partial x_{i \gamma} / \partial s=-c \partial v / \partial x_{1 \gamma}
$$

where $c=1 / \sqrt{\sum_{1 \gamma}\left(\partial v / \partial x_{i \gamma}\right)^{2}}$, which is the set of $3 N$ differential equations (aq. II-5) we introduced to define the reaction path. Because the formulation is in tems of Cartesian coordinates and is related to classical mechanfcs, no ambiguities or mathematical difficulties artse. 
Appendix B. Initial Conditions For a Diatonic Molecule

To classically determine probabilities, it is necessary to average over initial conditions. For an isclated diatomic molscule, one can change vari les to action-angle variables, $\left(N, Q_{v}\right),\left(J, Q_{J}\right)$, and $\left(M, Q_{M}\right)$ such that $\dot{N}_{v}=\dot{J}=\dot{M}=0$, with $N_{v}$ being the vibrational action, $J$ the rotational action or argular momentum, and $M$ being the projection of the angular momentum onto the $z$ axis. These variables allow a connection with quantum mechanical states to be made easily 57 . The probability $P$ of some event may be obtained by averaging over the initial angle variables $Q_{v}, Q_{J}, Q_{M}$, for $f$ ixed $\mathrm{N}_{v}, J$, and $M$,

$$
P=(2 \pi)^{-3} \int_{0}^{2 \pi} d Q_{V} \int_{0}^{2 \pi} d Q_{J} \int_{0}^{2 \pi} d_{M} x_{N} J M\left(Q_{v}, Q_{J}, Q_{M}\right) \quad B I
$$

where $x=1$ if the event occurs and 0 if it does not occur for the given initial conditions. Usually, the angular momentum is randoorly oriented in space, so an average may be taken over $M$ :

$$
\overline{\mathrm{P}}=\int_{-J}^{J} \mathrm{dM} P \int_{-J}^{J} d M=\frac{1}{2 J} \int_{-J}^{J} \text { ar } \mathrm{P} \quad B 2
$$

To do the Monte-Carlo integration ${ }^{45}$, the variables of integration are changed to $\xi$, wich $0: \xi \leq 1$, such that 


$$
\begin{aligned}
& 2 \xi_{1}-1=M / J=\lambda \\
& 2 \pi \xi_{2}=Q_{v} \\
& 2 \pi \xi_{3}=Q_{J} \\
& 2 \pi \xi_{4}=Q_{M}
\end{aligned}
$$

Eq. B2 becomes

$$
\bar{P}=\lim _{N \rightarrow \infty} \frac{1}{N} \frac{\sum_{\xi}^{N}}{\xi} X_{N} J M^{(\vec{\xi})}
$$

That is, one averages $x$ over $N$ randon evaluations of $\xi$ (each component of $\xi$ is taken to be a pseudorandom number for a given evaluation)

Approximate relations between the action-angle variables and ordinary molecular coordinates have been given by Porter, Raff, and Miller 57 for a rotating Morse oscillacor. The orbits given by them for $\theta$ and $\phi$ are not strictly correct. The corrected orbits are

$$
\begin{array}{lll}
r(t)=r_{e}-\frac{1}{a} \ln \left\{(-2 a)\left[b+\sqrt{b^{2}-4 a c} \sin \left(\omega_{N} t+Q_{N}\right)\right]\right. & B 5 a \\
\theta(r)=\arccos \left[\sqrt{1-\lambda^{2}} \cdot \cos \left(\omega_{J} t+Q_{J}+\operatorname{sign}\left(P_{r}\right) j \Delta_{J}\right)\right] & B 5 b \\
\phi(t)=Q_{M}+\operatorname{sign}\left(P_{\theta}\right) \arccos \left(\frac{\lambda \cot (\theta(t)]}{\sqrt{1-\lambda^{2}}}\right) & B 5 c
\end{array}
$$

where the formulae for $a, b, c, w_{N}, w_{J}$, and $\Delta_{J}$ may be found in Ref. 57 . The errors in the angular orbits rose from omission of a $\operatorname{sign}\left(P_{\tau}\right)$ and a sign $\left(\mathrm{p}_{\theta}\right)$ factor in the generators $W_{r}$ and $W_{\theta}$, respectively (eqs. 8a and 8b of Ref. 57). Anocher slight error is in eqs. $30 \mathrm{~b}$ and 
$30 c$ of Ref. 57. Here, the factor $r^{2}$ should be replaced by the expansion for $r^{2}$ given by their eq. $S$.

Thus, to generate the initial conditions for a diatomic we first pick $\lambda, Q_{v}, Q_{J}$, and $Q_{M}$ randomly according to eqs. B3. Then, since the calculations are to be made in spherical coordinates, $\tau, \theta$, and $\phi$ are calculated from eqs. B5. $P_{r}$ and $P_{\theta}$ may be obtained by either ccnservation of energy and angular momentum, or by differentiation of eqs. 30 of Ref. 57. This procedure is completely equivalent to randomly orienting the rolecule and its angular momentum vector and picking only $r$ and $P_{r}$ from the action-angle variable focmulae, which is the more standard approach ${ }^{45 a}$. Thus, the present approach offers no technical advantage ovar the ordinary approach for most applications, including the present one, except when rotational angle variables play an important role, as in some semiclassical applications.

The vibrational action $\mathrm{N}_{\mathrm{V}}$ is calculated at a time $t$ from the approximate formula of Ref. 57 ,

$$
N_{v}=-\frac{h}{2}+\frac{\sqrt{2 \mu}}{a}\left(\frac{b}{2 \sqrt{-c}}-\sqrt{-a}\right)
$$

and only depends on the rolecular energy and angular momentum state, $J(J+h)=\left(p_{\theta}^{2}+p_{\phi}^{2} / \sin ^{2} \theta\right) . \quad N_{v}$ was also calculated numerically ( $t$ r m $\left.N_{v}=-h / 2+(2 \pi)^{-1} \phi p_{r} d r\right)$ as a check on eq. B6 and, for all $N_{v}$ and $J$ with $S: 10, N_{v}$ from eq. B6 is accurate to three significant figures. Thus, essentially no error is introduced by the use of eq. 86 . 
Appendix $C$. Fixed Points of the Perisd Advance Map

An approximate, but surprisingly accurate estimate of the period 1 fixed points is as follows. Starting from eq. IV-29 of the text,

$$
K=H_{0}\left(\hat{p}+n_{1}\right)-\varepsilon V_{1}\left(\hat{p}+n_{1}\right) \cos \hat{q}-\hat{p} \Omega,
$$

where $\hat{q}=q-\Omega t$ and $\dot{p}=n-n_{1}$, one then uses the harmonic oscillator 8 $x(n, q)$ to esilimate $V_{1}$, rather than the exact form eq. IV-26b. This is valid for small $\mathrm{n}$ and simplifies the calculations. Thus

$$
\begin{aligned}
v_{1}(n) & =(2 \pi)^{-1} \int_{-\pi}^{\pi} x(n, q) \cos q d q \\
& =(2 \pi)^{-1} \int_{-\pi}^{\pi} \sqrt{(2 n+1) / \mu \omega_{0}} \cos ^{2} q d q \\
& =\sqrt{(2 n+1) / \mu \omega_{0}} / 2 .
\end{aligned}
$$

Eq. $\mathrm{Cl}$ becomes

$$
K=H_{0}\left(\hat{p}+n_{1}\right)-(\varepsilon / 2) \sqrt{\left[2\left(\hat{p}+n_{1}\right)+1\right] / \mu w_{0}} \cos \hat{q}-\hat{p} \Omega .
$$

The equations of motion conslstent with the Hamiltonian above are

$$
\begin{aligned}
& \dot{\hat{\mathrm{p}}}=-(\varepsilon / 2) /\left[2\left(\hat{\mathrm{p}}+\mathrm{n}_{1}\right)+1\right] / \hbar \omega_{\mathrm{o}} \text { sinq } \\
& \dot{\hat{q}}=\omega\left(\hat{p}+n_{1}\right)-\frac{(\varepsilon / 2) \cos \dot{q}}{\sqrt{\omega \omega_{0}}\left[\frac{\left.\left.\hat{p}+n_{1}\right)+1\right]}{[2(\hat{p})}\right.}-\Omega
\end{aligned}
$$


For a fixed point, we require $\dot{\hat{p}}=\dot{\hat{q}}=0$. Replacing $\hat{\mathrm{p}}+\mathrm{n}_{1}$ by $\mathrm{n}$, and setting $t=0$ so that $\hat{q}=q$, we obtain the two equations

$$
\begin{aligned}
-(\varepsilon / 2) \sqrt{(2 n+1) / \mu \omega_{0}} \text { sinq } & =0 \\
\omega(n)-\frac{(\varepsilon / 2) \cos q}{\sqrt{\mu \omega_{0}(2 n+1)}}-\Omega & =0 .
\end{aligned}
$$

Eq. C5a is satisfied for $q=0$ and $\pi$. Inserting either of these into eq. C5b yields a simple one-dimenstoral root equation for $n$, which can be solved by iteration. With the parameters of sec. IVC, one finds the fixed points $(n, q)=(0.6218, \pi),(0.3610,0)$, and $(-0.4828,0)$, which are in remarkably good agreement with the more exact fixed points, which were found to be $(0.61 i j, \pi),(0.3603,0)$, and $(-0.4321,0)$. 


\section{References}

1. See, for example, (a) M.A.D. Fluendy and K.P. Lawley, "Chemical Applications of Molecular Beam Scatcering", (Chapman and Hall Ltd., Lcndon, 1973), and (b) R.D. Levine and R.B. Bernstein, "Molecular Reaction Dynamics", (Clarendon Press, Oxford, 1974).

2. An excellent but somewhat outdated review of classical trajectory applications is (a) R.N. Porter, Ann. Rev. Phys. Chem, 25, 317 (1974). A more recent discussion is (b) R.B. Walker and J.C. Light, Ann. Rev. Phys. Chem. 31,401(1980).

3. M. Karplus, R.N. Porter, and R.D. Sharma, J. Chem. Phys. 43, $3259(1965)$.

4. (a) G.C. Schatz and A. Kuppermann, J. Chem. Phys. 62,2502 (1975).

(b) A.B. Elkowitz and R.E. Wyact, J. Chem. Phys. 62,2504 (1975).

(c) A.B. Elkowitz and R.E. Wyatt, J. Chem. Phys. 63,702(1975).

(d) G.C. Schatz and A. Kuppermann, J. Chem. Phys. 65,4668(1976).

5. (a) J.T. Muckerman, J. Chem. Phys. 54,1155(1971).

(b) J.T. Muckerman, J. Chem. Phys. 56,2997(1972).

(c) J.T. Muckerman, J. Chem. Phys. 57,3388(1972).

6. W.L. Hase, R.J. Wolf, C.S. Sloane, J. Chem. Phys. 71,2911(1979).

7. R.E. Wyatt, in "Aton - Molecule Collision Theory, A Guide fot the Experimentalist", edited by R.B. Bernstein, (Plenum, New Yorik, 1979), pp. 567-94. 
8. W.H. Miller, Adv. Chem. Phys. 25,69(1974).

9. For a good treatment of the WKB approximation, see (a) E. Merzbacher, "Quantum Mechanics", (Wiley, New York, 1970), and (b) D. Rapp, "Quantum Mechanics", (Holt, Rinehart, and Winston, New York, 1971).

10. W.H. Miller, N.C. Handy, and J.E. Adiss, J. Chem. Phys. 72, $99(1980)$.

11. W.H. Miller, J. Am. Chem. Soc. 101,6810(1979).

12. (a) W.H. Miller and C.W. MCCurdy, J. Chem. Phys. 69,5163(1978).

(b) H-D. Meyer and W.H. Miller, J. Chem. Phys. 70,3214(1979).

(c) H-D. Meyer and W.H. Miller, J. Chem. Phys. 71,2156(1979).

(d) H-D. Meyer and W.H. Miller, J. Chem. Phys. 22,2272(1980).

13. V.I. Arnold, "Mathematical Methods of Classical Mechanics", (Springer-Verlag, New York, 1978), p. 114.

14. B.V. Chirikov, Phys. Rep. 52,263(1979)

15. P.J. Robinson and K.A. Holbrook, "Unimolecular Reactions", (Wiley, New York, 1972).

16. W.L. Hase, in "Dynamics of Molecular Collisions, Part B", ediced by W.H. Miller, (Plenum, New York, 1976), pp.121-69.

17. W. Forst, "Theory of Unimolecular Reaciions", (Academic, New York, 1973), 9.61 . 
18. H.S. Johnston, "Gas Phase Reaction Rate Theory", (Ronalò Press, New York, 1966), Pp.37-47.

19. (a) P. Pulay, in "Applications of Electronic Structure Theory", edited by H.F. Schaefer, (Plenum, New York,1977), pp.153-85. For details on analytic configuration interaction dexivatives, see (b) B.R. Brooks, W.D. Latdig, P. Saxe, J.D. Goddard, Y. Yamaguchi, and H.F. Schaefer, J. Chem. Phys. 72,4652(1980), and (c) R. Krishnan, H.B. Schlegal, and J.A. Pople, J. Chem. Phys. 72,4654(1980).

20. See, for example, T. Takada, M. Dupuis, and H.F. King, J. Chem. Phys. 75, 332(1981), and references therein.

21. C.J. Cerjan and W.H. Miller, J. Chem. Phys. 75,2800(1981).

22. Y. Osamura, H.F. Schaefer, S.K. Gray, and W.H. Miller, J. Am. Chem. Soc. 103,1904(1981). See also Y. Osamura and H.F. Schaefer, Chem. Phys. Letr. 79,412(1931).

23. (a) P.S. SkeIl and J.H. Plonka, J. Ar. Chem. Soc. 92,5620(1970); P.S. Skeli, F.A. Fagone, and K.J. K.Iaburde, J. Am. Chem. Soc. 94,7862 (1972); P.S. Skell, J.J. Havel, and M.J. McGlinchey, Acc. Chem. Res. 6, $97(1973)$.

(b) C. Reiser, F.M. Lussier, C.C. Bonsen, and J.I. Steinfeld, J. Am. Chem. Soc. 101,350(1979); C. Reiser and J.I. Steindeld. J. Phys. Chem. $\underline{84}, 680(1980)$.

24. R. K.rishnan, M.J. Frisch, J.A. Pople, and P.V.R. Schleyer, Chem. Phys. Lett. 79,408(1981). 
25. M. Born, "The Mechanics of the Atom", (Ungar, New York, 1960),pp. 249-56.

26. See, for example, S. Chapman, B.C. Garrett, and W.H. Miller, J. Chem. Phys. 64, 502(1976).

27. S.K. Gray, W.H. Miller, Y. Yamaguchi, and H.F. Schaefer, J. Chem. Phys. 23,2733(1980).

28. See, for example, F.B. Hildebrand, "Introduction to Numerical Analysis", (McGraw-Hill, New York, 1956), Chap. 6.

29. K. Ishida, K. Morokuma, and A. Komornicki, J. Chem. Phys, 66, $2153(1977)$.

30. A good introduction to formaldehyde dissociation is the review article, W.M. Celbart, M.I. Elert, and D.F. Heller, Chem. Rev. $80,403(1980)$.

31. J.C. Weisshaar and C.8. Moore, J. Chem. Phys. 20,5135(1979). 32. J.D. Goddard and H.F. Schaefer, J. Chem. Phys. 70,5117(1979). 33. J.D. Goddard, Y. Yamaguchi, and H.F. Schaefer, J. Chem. Phys. 75. $3459(1981)$.

34. S.K. Gray, W.H. Miller, Y. Yamaguchi, and H.F. Schaefer, J. An. Chem. Soc. 103,1900(1981).

35. J.L. Duncan and P.D. Mallison, Chem. Phys, Lett. 23,597(1973). 
36. B.A. Waite, W.H. Miller, and S.K. Gray, to be published; see also B.A. Waite and W.H. Miller, J. Chem. Phys, 76, 2412(1982).

37. M.J. Frisch, R. Krishnan, and J.A. Pople, J. Phys. Cherr. 85, $1467(1981)$.

38. H.F. King, M. Dupuis, and J. R.ys, National Resource for Computation in Chemistry Software Catalog, Vol.1, Program No. QHO2 (HONDO), 1980.

39. K. Yamashita, T. Yambe, and K. Fukui, Chem. Phys. Lett. $\underline{84}, 123(1981)$.

40. See, e.g., K. Lacman, Adv. Chem. Phys. 42,513(1980).

41. M.R. Faist and R.D. Levine, J. Chem. Phys. 64,2953(1976).

42. (a) J.C. Tully and R.K. Preston, J. Chem. Phys. 55, 562(1971).

(b) J.R. Krenos, R.K. Preston, R. Wolfgang, and J.C. Tully, J. Chem. Phys. 60,1634(1974).

43. W.H. Miller, J. Chem. Phys. 68,4431(1978).

44. F.T. Smith, Phys. Rev. 179,111(1969); see also M. Baer, Chem. Phys. Lett. 35,113(1975).

45. (a) R.N. Porcer and L.M. Raff, in "Dynamics of Molecular Collisions", edited by W.H. Miller, (Plenum, N.Y.,1976),P.1.

(b) D.L. Burker, Meth. Comp. Phys. 10,287(1971).

46. A.E. Orel, D.P. Ali, and W.H. Miller, Chem. Phys. Lete. 79,137 (1981) 
47. W.H. Miller and F.T. Smith, Phys. Rev. 17A,939(1978).

48. See the initial value representation, discussed in W.H. Mfller, J. Chem. Phys. 53, 3578(1970).

49. See, e.g., M.S. Child, "Molecular Collision Theory", (Academic, N.Y.,1974), PP. 161-79.

50. See, for example, papers in "Advances in Laser Chemistry", ediled by A.H. Zewail, (Springer, N.Y.,1978) and "Laser-Induced Processes in Molecules", edited by K.L. Kompa and S.D. Smith, (Plenum, N.Y.,1978).

51. (a) D. Poppe, Chem. Phys. 45, 371(1980).

(b) D.W. Noid, C. Bottcher, and M.L. Koszykowsk1, Chem. Phys. Lett. $72,397(1980)$.

(c) D.W. Notd, M.L. Koszykowski, R.A. Marcus, and J.D. MacDonald, Chem. Phys. Lett. 51,540(1977).

52. R.B. Walker and R.K. Preston, J. Chem. Phys. 67,2017(1977).

53. (a) D.W. Noid and J.R. Stine, Chem. Phys. Lett. 65, 153(1979).

(b) J.R. Stine and D.W. No1d, Chem. Phys. lerr. 77,287(1981).

(c) K.M. Christoffel and J.M. Bowman, J. Phys. Chem. 85,2159(1981).

54. (a) S.C. Leasure and R.E. Wyatt, Opt. Eng. 19,46(1980).

(b) S.C. Leasure and R.E. Wyatt, Chem. Phys. Lett. 61,625(1979).

(c) S.C. Leasure, K.F. Milfeld, and R.E. Wyatt, J. Chem. Phys. 74, $6197(1981)$.

55. W.H. Flygare, "Molecular Structure and Dynamics", (Prent1ce-Hall, Englewood Cliffs, N.J., 1978),p.11. 
56. W.H. Miller, J. Cilem. Phys. 69,2188(1978).

57. R.N. Porter, L.M. Raff, and W.H. Miller, J. Chem. Phys. 63, $2214(1975)$.

58. (a) M.L. Sage, Chem. Phys. 35,375(1978).

(b) R. Wallace, Chem. Phys. Lett. 37,125(1976).

59. L.I. Shampine and M.K. Gordan, "Computer Solution of Ordinary Differential Equations : The Initial Value Problem", (Freeman, S.F., 1975). The actual program used was ODE from the SANDIA Laboratory.

60. C.W. Gear, SIAM Rev. 23,10(1981).

61. (a) M. Sargent, M.O. Scully, and W.E. Lamb, "Laser Physics", (Addison-Wesley, Reading, MA,1974).

(b) S. Flugge, "Practical Quantum Mechanics", (Springex,N.Y., 1971), Vo1. II,P. 138 .

(c) M. Quack, J. Chem. Phys, 69,1282(1978).

62. J.V. Moloney and W.J. Meath, Mol. Phys. 31,1537(1976).

63. P.S. Dardi and S.K. Gray, J. Chem. Phys., to be published.

64. M.V. Berry, N.L. Balazs,M. Tabor, and A. Voros, Ann. Phys. 122, $26(1979)$

65. H.T. Davis, "Introduction to Nonlinear Differential and Integral Equations", (Dover, N.Y., 1962).

66. (a) L.F. Shampine and R. C. Allen, "Numerical Compuring : An. Introduction", (Saunders, Philadelphia, 1973). 
66. (b) D.R. McLaughlin and D.L. Thompson, J. Chem. Phys. $\underline{59}$, 4393(1973); see also N. Sathyamurthy and L.M. Raff, J. Chem. Phys. 63, 464(1975).

67. C.C. Rankin and W.H. Miller, J. Chem. Phys. 55,3150(1971), Note that their phase convention for $q$ differs by $\pi$ from ours and their energy is with respect to the separated atoms as zero.

68. See, e.g., M.V. Berry in "Topics in Nonlinear Dynamics", ediced by S. Jorna, (AIP, N.Y., 1978), Chapter 2.

69. H. Goldstein, "Classical Mechanics", (Addison-Wesley, Reading, MA, 1950).

70. I.S. Gradshety.. and I.M. Ryzhik, "Table of Integrals, Series, and Products", (Academic, N.Y., 1965).

71. M.J. Davis and R.E. Wyatt, Chem. Phys. Lett. 86.235(1982).

72. D.W. Oxtoby and S.A. Rice, J. Chem. Phys. 65, 1676(1976).

73. Robert E. Wyatt, personal communication.

74. E.A. McCullough and D.M. S1lver, J. Chem. Phys. 62,4050(1975)

75. (a) K. Fukui, J. Phys. Chem. 74,4161(1970).

(b) H.F. Schaefer, Chem. Brit. 11,227(1975). 
Table I. Predicted DZ+P CI harmonic vibrational frequencies for vinylidene, acetylene, and the transition state connecting therd.

\begin{tabular}{|c|c|c|}
\hline Species & Frequency & $\begin{array}{c}\begin{array}{c}\text { Normal } \\
\text { Mode } \\
\text { Description }\end{array} \\
\end{array}$ \\
\hline \multirow{6}{*}{ vinylidene } & 3344 & $\mathrm{CH}$ asym. stretch \\
\hline & 3239 & CH sym. stretch \\
\hline & 1710 & CC stretch \\
\hline & 1288 & $\mathrm{CCH}$ bend \\
\hline & 787 & out of plane \\
\hline & 444 & $\mathrm{CH}_{2}$ rock \\
\hline \multirow{6}{*}{ transitton state } & 3454 & $\mathrm{CH}_{\mathrm{a}}$ stretch \\
\hline & 2699 & $\mathrm{CH}_{b}$ stretch \\
\hline & 1874 & CC stretch \\
\hline & 937 & HCC bend \\
\hline & 573 & out of plan \\
\hline & $1029 i$ & reaction coordinate \\
\hline \multirow{5}{*}{ acetylene } & 3583 & $\mathrm{CH}$ asym. stretch \\
\hline & 3488 & $\mathrm{CH}$ sym. stretch \\
\hline & 2073 & CC stretch \\
\hline & 764 & $\mathrm{CCH}$ bend \\
\hline & 610 & $\mathrm{CCH}$ bend \\
\hline
\end{tabular}


Table II. Harmonic Vibratiuna' Frequencies af $\mathrm{H}_{2} \mathrm{CO}$.

$\begin{array}{cccccc} & \text { DZ-SCF } & \text { DZP-SCF } & \text { DZ-CI } & \text { DZP-CI } & \text { EXP } \\ \omega_{1}\left(\mathrm{a}_{1}\right) & 3223 & 3149 & 3028 & 3074 & 2944 \\ \omega_{2}\left(\mathrm{a}_{1}\right) & 1878 & 2006 & 1703 & 1869 & 1764 \\ \omega_{3}\left(\mathrm{a}_{1}\right) & 1651 & 1656 & 1544 & 1596 & 1563 \\ \omega_{4}\left(b_{1}\right) & 1324 & 1335 & 1194 & 1243 & 1191 \\ \omega_{5}\left(b_{2}\right) & 3315 & 3226 & 3112 & 3155 & 3009 \\ \omega_{6}\left(b_{2}\right) & 1349 & 1307 & 1263 & 1306 & 1287\end{array}$

Units for Erequencies are $\mathrm{cin}^{-1}$.

barious column headings refer co the various ab inicio basis sers and extent of configuration interaction included in the calculations.

$c^{c}$ The experimental harmonic fregrencies given in reference 35 . 
Iable III. Transition State Parameters ${ }^{a}$ for $\mathrm{H}_{2} \mathrm{CO}+\mathrm{H}_{2}+\mathrm{CO}$.

\begin{tabular}{|c|c|c|c|c|c|}
\hline & $D Z-5 C E$ & DZP-SCF & $\underline{D Z-C I}$ & D2P-CI & SCALED $^{\mathrm{C}}$ \\
\hline$w_{1}\left(a^{\prime}\right)$ & 3156 & 3243 & 3159 & 3263 & 3125 \\
\hline$w_{2}\left(a^{\prime}\right)$ & 1948 & 2092 & 1764 & 1939 & 1830 \\
\hline$w_{3}\left(a^{\prime}\right)$ & 1371 & 1526 & 1310 & 1555 & 1523 \\
\hline$\omega_{4}\left(a^{\circ}\right)$ & 800 & 829 & 803 & 876 & 839 \\
\hline$w_{S}\left(a^{\prime}\right)$ & $2320 i$ & $2305 i$ & 19971 & 21241 & $2026 i$ \\
\hline$w_{6}\left(a^{\prime \prime}\right)$ & 1015 & 1024 & 889 & $\sim 950$ & 936 \\
\hline$v_{0}\left(\frac{k c a l}{\text { mole }}\right)^{d}$ & 113.7 & 105.9 & 100.3 & 98.1 & $92^{\mathrm{e}}$ \\
\hline
\end{tabular}

\footnotetext{
$a, b$ See Table II.

c Scaled Erequencies, see text.

d Barrier helght relative to vibrationless $l_{2} \mathrm{CO}$.

e The best estimates of Goddard et al. (reference 33 ) of the classical bartier, with a probable error of $\pm 1 \mathrm{kcal} / \mathrm{mole}$.
} 
Table IV. Rate Constants via Simple RRKM + Tunneling Model.

\begin{tabular}{|c|c|c|c|c|c|}
\hline \multirow[b]{2}{*}{$\underline{E}-V_{0}$ (kcal/mole) } & \multicolumn{5}{|c|}{$\log k\left(\sec ^{-1}\right)$} \\
\hline & DZ-SCF & DZP-SCF & $\underline{\mathrm{D} 2-\mathrm{CI}}$ & $D Z P-C I$ & SCALED \\
\hline-2 & 3.57 & 3.41 & 2.91 & 2.99 & 2.95 \\
\hline 0 & 4.41 & 4.26 & 3.90 & 3.92 & 3.92 \\
\hline 2 & 5.24 & 5.10 & 4.87 & 4.83 & 4.88 \\
\hline 4 & 6.06 & 5.93 & 5.83 & 5.74 & 5.81 \\
\hline 6 & 6.88 & 6.76 & 6.77 & 6.63 & 6.75 \\
\hline 8 & 7.68 & 7.57 & 7.71 & 7.51 & 7.67 \\
\hline 10 & 8.43 & 8.34 & 8.57 & 8.36 & 8.55 \\
\hline 12 & 9.02 & 9.00 & 9.17 & 9.05 & 9.21 \\
\hline
\end{tabular}


Table V. Frequency Variation along the Reaction Path.

\begin{tabular}{|c|c|c|c|}
\hline & $=0.21$ & 0 & +0.21 \\
\hline$\omega_{1}$ & 3517 & 3263 & 2642 \\
\hline$w_{2}$ & 1932 & 1939 & 1981 \\
\hline$w_{3}$ & 1594 & 1555 & 1648 \\
\hline$\omega_{4}$ & 953 & 876 & 738 \\
\hline$\omega_{6}^{a}$ & $(2950)$ & $\approx 950$ & $(\approx 950)$ \\
\hline
\end{tabular}

a The out-of-plane bending frequency. 
Table VI. Effect on Rate of More Rigorous Dynamical Model.

\begin{tabular}{|c|c|c|c|c|c|}
\hline$E-V_{0}(\mathrm{kcal} / \mathrm{mole})$ & A & $\mathbf{B}$ & C & D & $E$ \\
\hline-2 & 2.99 & 3.14 & 3.17 & 3.14 & 2.98 \\
\hline 0 & 3.92 & 4.00 & 4,03 & 4.00 & 3.86 \\
\hline 2 & 4.83 & 4.86 & 4.89 & 4.86 & 4.74 \\
\hline 4 & 5.74 & 5.72 & 5.75 & 5.72 & 5.62 \\
\hline 6 & 6.63 & 6.58 & 6.61 & 6.58 & 6.50 \\
\hline 8 & 7.51 & 7.50 & 7.52 & 7.49 & 7.41 \\
\hline 10 & 8.36 & 8.34 & 8.35 & 8.33 & 8.25 \\
\hline 12 & 9.05 & 9.04 & 9.04 & 9.02 & 8.97 \\
\hline
\end{tabular}

${ }^{a}$ See text for a description of the theoretical models corresponding to columns A-E. 
Table VII. Comparison of semiclassical perturbation theory (SCL) and exact quantum (QM) probabilities for the Landau-Zener model.

$\begin{array}{lll}Z & P_{\left.1+0^{(S C L}\right)} & P_{1+0^{(Q M)}} \\ 0 . & 0 . & 0 . \\ 0.2 & 0.0099 & 0.0099 \\ 0.4 & 0.0384 & 0.0392 \\ 0.6 & 0.0822 & 0.0861 \\ 0.8 & 0.1360 & 0.1479 \\ 1.0 & 0.1937 & 0.2212 \\ 1.2 & 0.2483 & 0.3023 \\ 1.4 & 0.2936 & 0.3874 \\ 1.6 & 0.3248 & 0.4727 \\ 1.8 & 0.3381 & 0.5551 \\ 2.0 & 0.3326 & 0.6321 \\ \infty & 0 . & 1 .\end{array}$


Table VIII. Some relevant energy levels for HF, according to the rotating Morse oscillator approximation.

$\begin{array}{llll}v & j & & \\ 0 & 0 & 0.0093309 & 2048 \\ 0 & 1 & 0.0095187 & 2089 \\ 0 & 2 & 0.0098941 & 2171 \\ 1 & 0 & 0.0274001 & 6014 \\ 1 & 1 & 0.0275819 & 6054 \\ 1 & 2 & 0.0279454 & 6133 \\ 2 & 0 & 0.0446793 & 9806 \\ 2 & 1 & 0.0448551 & 9845 \\ 2 & 2 & 0.0452065 & 9922\end{array}$


Table IX. Approximate cime averaged probabilities for vibrational cransicions of $\mathrm{HF}$ in a $1.0 \mathrm{TW} / \mathrm{cm}^{2}$ laser.

\begin{tabular}{|c|c|c|c|c|c|c|}
\hline \multirow[b]{2}{*}{$\bar{v}\left(\mathrm{~cm}^{-1}\right)$} & \multicolumn{3}{|c|}{ non-rotacing } & \multicolumn{3}{|c|}{ rotating } \\
\hline & $\mathrm{P}_{0}$ & $P_{1}$ & $\mathrm{P}_{2}$ & $\mathrm{P}_{0}$ & $P_{1}$ & $\mathrm{P}_{2}$ \\
\hline \multirow[t]{2}{*}{3850} & $0.88(\mathrm{QM})$ & 0.08 & 0.04 & 0.99 & 0.01 & 0.00 \\
\hline & $1.00(\mathrm{CL})$ & 0.00 & 0.00 & & & \\
\hline \multirow[t]{2}{*}{3879} & 0.47 & 0.08 & 0.45 & 0.53 & 0.03 & 0.44 \\
\hline & 0.88 & 0.12 & 0.00 & 0.99 & 0.01 & 0.00 \\
\hline \multirow[t]{2}{*}{3900} & 0.83 & 0.11 & 0.06 & 0.96 & 0.03 & 0.01 \\
\hline & 0.73 & 0.19 & 0.08 & 0.94 & 0.04 & 0.02 \\
\hline \multirow[t]{2}{*}{3937} & 0.69 & 0.28 & 0.03 & 0.47 & 0.07 & 0.46 \\
\hline & 0.69 & 0.24 & 0.06 & 0.67 & 0.27 & 0.06 \\
\hline \multirow[t]{2}{*}{3966} & 0.51 & 0.47 & 0.02 & 0.87 & 0.12 & 0.01 \\
\hline & 0.63 & 0.36 & 0.01 & 0.58 & 0.40 & 0.02 \\
\hline \multirow[t]{2}{*}{4006} & 0.69 & 0.30 & 0.01 & 0.50 & 0.49 & 0.01 \\
\hline & 0.68 & 0.32 & 0.00 & 0.66 & 0.34 & 0.00 \\
\hline \multirow[t]{2}{*}{4085} & 0.93 & 0.07 & 0.00 & 0.95 & 0.05 & 0.00 \\
\hline & 0.90 & 0.10 & 0.00 & 0.88 & 0.12 & 0.00 \\
\hline
\end{tabular}


Table $X$. Approximate time averaged vibrational transition probabilicies Eor rotating $\mathrm{HF}$ in a $2.5 \mathrm{TW} / \mathrm{cm}^{2}$ laser. (a)

\begin{tabular}{llll}
$\bar{v}\left(\mathrm{~cm}^{-1}\right)$ & $P_{0}$ & $P_{1}$ & $P_{2}$ \\
\hline 3879 & $0.51(\mathrm{QM})$ & 0.07 & 0.42 \\
& $0.88(\mathrm{CL})$ & 0.07 & 0.05 \\
3900 & 0.90 & 0.05 & 0.05 \\
& 0.67 & 0.17 & 0.16 \\
3937 & 0.48 & 0.10 & 0.42 \\
3966 & 0.77 & 0.31 & 0.19 \\
& 0.52 & 0.18 & 0.05 \\
4006 & 0.52 & 0.39 & 0.09 \\
4085 & 0.89 & 0.45 & 0.03 \\
& 0.61 & 0.37 & 0.02 \\
& 0.78 & 0.20 & 0.00
\end{tabular}

(a)

The classical results shown for $\bar{v}=3879$ and $3937 \mathrm{~cm}^{-1}$ were actually run at 3870 and $3927 \mathrm{~cm}^{-1}$, respectively. The probabilities will not vary much since the classical peak is broad. It was displayed in the cable this way to avoid confusion since the overall trends are still clear. 
TableXI. Quantum mechanical transition probabilities and energy absorbed as a function of pulse time for laser phases of 0 and $\pi / 2$ at $\omega=4006 \mathrm{~cm}^{-1}$ and $I=1.0 \mathrm{TW} / \mathrm{cm}^{2}$.

\begin{tabular}{|c|c|c|c|c|c|c|}
\hline$E(p s)$ & $\delta \stackrel{0}{=} 0$ & $\delta=\pi / 2$ & $\delta=0$ & $\delta=\pi / 2$ & $\begin{array}{l}\angle \mathrm{E}(\mathrm{c} \\
\delta \stackrel{r}{=}\end{array}$ & $\begin{array}{l}(a \cdot u .) \\
\delta=\pi / 2\end{array}$ \\
\hline 0.0 & 1.00 & 1.00 & 0.00 & 0.00 & 0.0000 & 0.0000 \\
\hline 0.4 & 0.30 & 0.32 & 0.63 & 0.66 & 0.0125 & 0.0126 \\
\hline 0.8 & 0.13 & 0.14 & 0.81 & 0.83 & 0.0156 & 0.0158 \\
\hline 1.2 & 0.94 & 0.95 & 0.05 & 0.05 & 0.0010 & 0.0009 \\
\hline 1.6 & 0.51 & 0.53 & 0.44 & 0.45 & 0.0084 & 0.0086 \\
\hline 2.0 & 0.03 & 0.03 & 0.92 & 0.92 & 0.0177 & 0.0179 \\
\hline 2.4 & 0.81 & 0.81 & 0.17 & 0.17 & 0.0034 & 0.0033 \\
\hline 2.8 & 0.73 & 0.73 & 0.24 & 0.24 & 0.0047 & 0.0048 \\
\hline 3.2 & 0.01 & 0.01 & 0.95 & 0.94 & 0.0180 & 0.0182 \\
\hline 3.6 & 0.62 & 0.62 & 0.36 & 0.36 & 0.0068 & 0.0069 \\
\hline 4.0 & 0.88 & 0.90 & 0.09 & 0.09 & 0.0018 & 0.0017 \\
\hline 4.4 & 0.08 & 0.09 & 0.87 & 0.88 & 0.0167 & 0.0167 \\
\hline
\end{tabular}


Table XII. Quantum mechanical transition probabilities and energy absorbed as a function of pulse times for laser phases of 0 and $\pi / 2$ at $\omega=3937 \mathrm{~cm}^{-1}$ and $I=1.0 \mathrm{TW} / \mathrm{cm}^{2}$.

\begin{tabular}{|c|c|c|c|c|c|c|}
\hline$c(p s)$ & $\delta=0$ & $\delta=\pi / 2$ & $\delta=0$ & $\delta=\pi / 2$ & $\begin{array}{l}\langle E(t) \\
\delta=0\end{array}$ & $\mathrm{M}_{\delta=\pi / 2}^{(\text {a.u. })}$ \\
\hline 0.0 & 1.00 & 1.00 & 0.00 & 0.00 & 0.0000 & 0.0000 \\
\hline 0.4 & 0.93 & 0.93 & 0.05 & 0.05 & 0.0021 & 0.0019 \\
\hline 0.8 & 0.78 & 0.80 & 0.19 & 0.19 & 0.0072 & 0.0071 \\
\hline 1. 2 & 0.57 & 0.58 & 0.37 & 0.38 & 0.0142 & 0.0144 \\
\hline 1.6 & 0.36 & 0.37 & 0.57 & 0.57 & 0.0218 & 0.0219 \\
\hline 2.0 & 0.18 & 0.18 & 0.71 & 0.74 & 0.0282 & 0.0284 \\
\hline 2.4 & 0.05 & 0.05 & 0.83 & 0.81 & 0.0320 & 0.0324 \\
\hline 2.8 & 0.00 & 0.00 & 0.83 & 0.86 & 0.0333 & 0.0338 \\
\hline 3.2 & 0.03 & 0.03 & 0.80 & 0.80 & 0.0322 & 0.0324 \\
\hline 3.6 & 0.14 & 0.13 & 0.69 & 0.71 & 0.0283 & 0.0287 \\
\hline 4.0 & 0.27 & 0.29 & 0.56 & 0.57 & 0.0231 & 0.0233 \\
\hline 4.4 & 0.47 & 0.47 & 0.40 & 0.40 & 0.0169 & 0.0170 \\
\hline
\end{tabular}


Table XIII Root mean square deviations between the map predictions and direct numerical integration of crajectories. The resules are for 50 trajectories with $\mathrm{n}_{0}=0$ inicially. Typical values of $p$ and $x$ are \pm 3 and 2 a.u. respectively.

R.M.S. deviation

\begin{tabular}{ccc}
$r$ & $P$ & $x$ \\
\hline 100 & 0.005 & 0.0001 \\
200 & 0.008 & 0.0003 \\
300 & 0.016 & 0.0010 \\
400 & 0.024 & 0.0020 \\
500 & 0.078 & 0.0029
\end{tabular}




\section{Figure Captions}

Figure 1. Stationary point geometries at the DZP-CI level of theory for vinylidene, acetylene, and the transition state connecting them.

Figure 2. Rate of vinylidene earrangement to acetylene as a function of excess vibrat Lonal energy in vinylidene, i.e. vibrational energy in excess of the zero-point energy. The solid curve is for a classical barrier of $4 \mathrm{kcal} / \mathrm{mol}$, and che dashed curve is for a barrier of $6 \mathrm{kcal} / \mathrm{mol}$. Arrows on the energy axis indicate the respective classical thresholds.

Figure 3. Plot of the $x$ and $y$ coordinates of the $t$ atom along the reaction pach for $\mathrm{HNC} \rightarrow \mathrm{HCN}$. The origin of the coordinate system is the center of mass of $C N$. Only the region important for tunneling is shown, and the spurious oscillations have been smoothed out.

Figure 4. Potential energy (left hand scale) and vibrational frequencies (right hand scale) as a function of the reaction coordinate for HNC $\rightarrow$ HCN.

Figure 5. Vibrational mode coupling constants as a function of the reaction coordinate.

Figure 6. Microcanonical rate constant for zero cotal angular momentum for the reaction HNC $\rightarrow$ HCN. The clasjical thashold is indicated by an arrow. 
Figure 7. Potential energy profile along the formaldehyde reaction path for decomposition. The points are the ab initis (D2P-CI) results and the solid curve is an Eckart potential fit.

Figure 8. Potential energy profile, calculated at the DZ-SCF level of ab initio theory, for formaldehyde decomposition.

Figure 9. Vibrational frequencles as a function of reaction coordinate for formaldehyde decomposition, calculated at the DZ-SCF level of theory.

Figure 10. Selected vibrational mode couplings for the formaldehyde reaction path. (a) $B_{12}$, (b) $B_{31}$, (c) $B_{32}$, See text for more discussion.

Figure 11. Classical (points), quantum (solid curve), and perturbative semiclassical (dashed curve) results for $\mathrm{Na}+\mathrm{I}^{+} \mathrm{Na}^{+}+\mathrm{I}^{-}$ cross section.

Figure 12. Classical and quantum mechantcal time averaged energy absorption for $\mathrm{HF}$ in a $1.0 \mathrm{TW} / \mathrm{cm}^{2}$ laser. (a) nonrotating $\mathrm{HF}$, (b) rotacing $\mathrm{HF}$.

Eigure 13. Classical and quantum mechanical time avisaged energy absorption for rotating $\mathrm{HF}$ in a $2.5 \mathrm{TW} / \mathrm{cm}^{2}$ laser.

igure 14. Time-dependent energy absorption for nonrotating HF with laser frequency $\bar{V}=3966 \mathrm{~cm}^{-1}$ and $I=1.0 \mathrm{TW} / \mathrm{cm}^{2}$. 
Figure 15. Time-dependent energy absorption for rotating HF with $\bar{v}=4006 \mathrm{~cm}^{-1}$ and $I=1.0 \mathrm{TW} / \mathrm{cm}^{2}$.

Figure 16. Quantum time-dependent energy absorption for rotating HF wich $\bar{U}=3937 \mathrm{~cm}^{-1}$ and $I=1.0 \mathrm{TW} / \mathrm{cm}^{2}$. Jaggedness is due to poor resolution of high frequency oscillacions.

Figure 17. Classical time-dependent energy absorption for rotating HF with $\bar{v}=3937 \mathrm{~cm}^{-1}$ and $I=1.0 \mathrm{TW} / \mathrm{cm}^{2}$.

Figure 18. Classical and quantur probabilities $\mathrm{p}_{v j}$ for hiF with $\vec{v}=4006 \mathrm{~cm}^{-1}$ and $I=1.0 \mathrm{TW} / \mathrm{cm}^{2}$.

Figure 19. Classical probabilicies for HF wich $\bar{v}=3937 \mathrm{~cm}^{-1}$ and $I=1.0 \mathrm{TW} / \mathrm{cm}^{2}$.

Figure 20. Quantum probabilities $P_{00}$ and $P_{22}$ for HF with $\vec{v}=3937 \mathrm{~cm}^{-1}$ and $I=1.0 \mathrm{TW} / \mathrm{cm}^{2}$.

Figure 21. Surface of section plots for the period advance map.

a) $p-x$ space. b) $n-q$ space. Open circles indicate stable fixed points and filled circles indicace unscable fixed points.

Figure 22. Evolution of an initial $n_{0}=1 / 2$ state. d) after $r=20$ periods, b) after 40 periods, c) after 60 periods. Note the dashed line in a) represents the inicial scace.

Figure 23. a) result of mapping an $n_{0}=0$ state 40 periods, b) result of mapping an $n_{0}=1$ state 40 periuds. 
Figure 24. Evolution of the entire $n-q$ phase plane. After a) 20 periods, b) 40 periods, and c) 60 periods. See text for details.

Figure 25. Pendulum model predictions for the fate of initial states a) $n_{0}=1 / 2$, b) $n_{0}=0$, and c) $n_{0}=1$, after 40 pertods. 

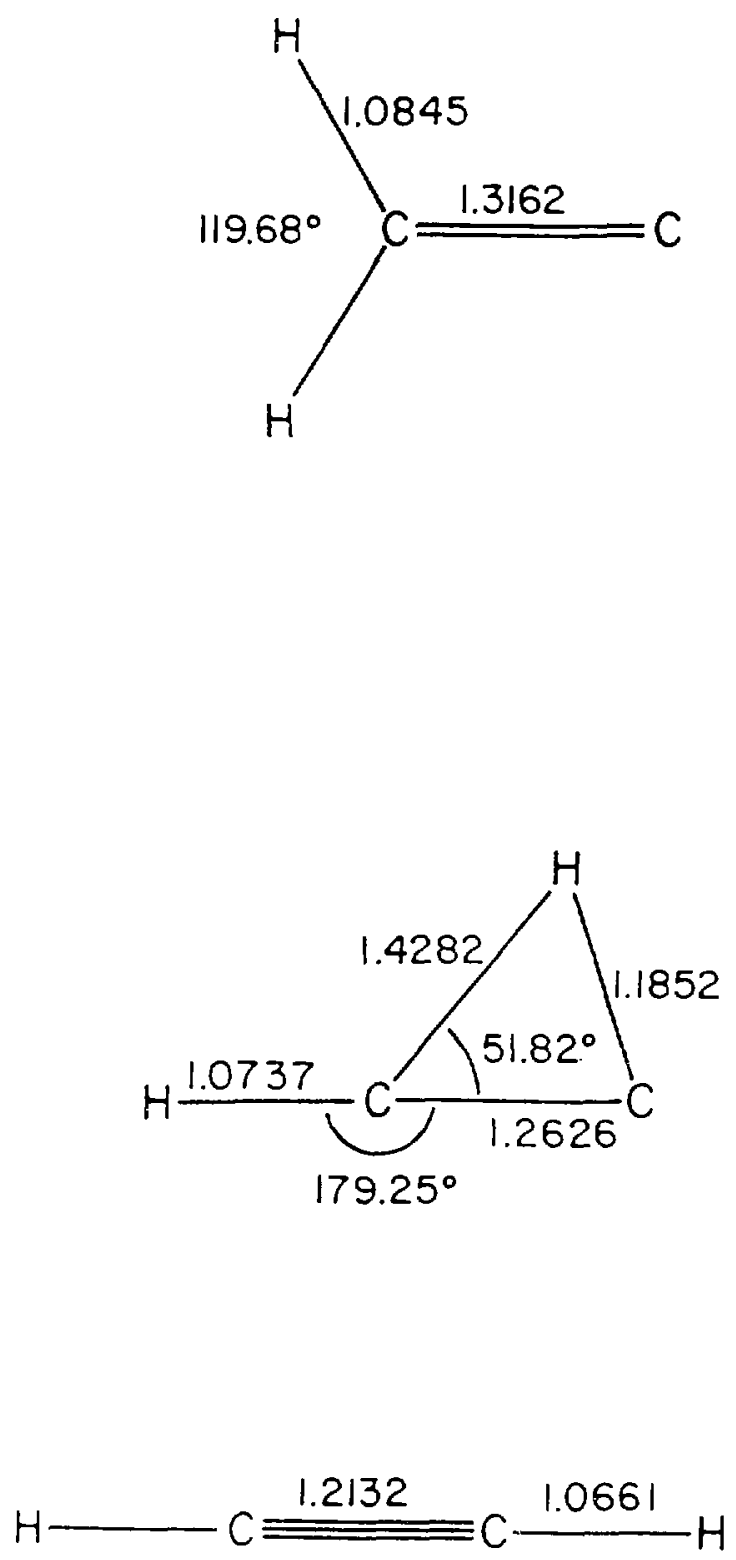

Figure ! 


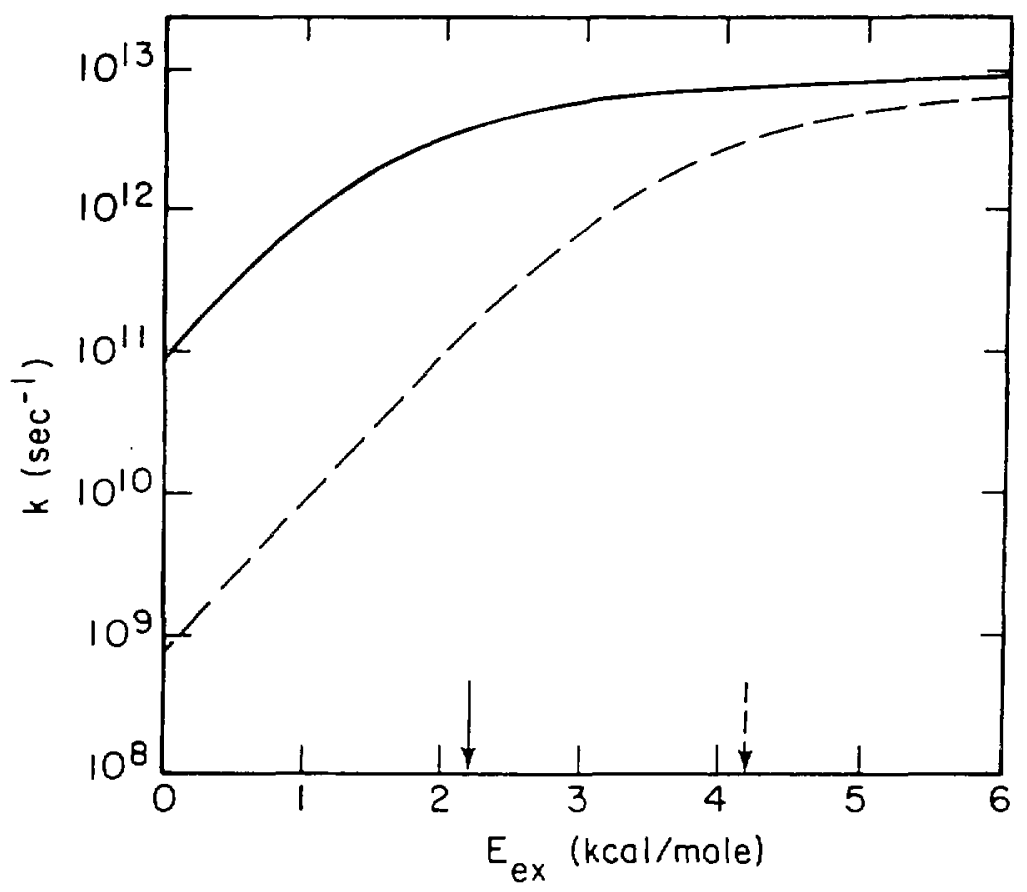

Figure 2 


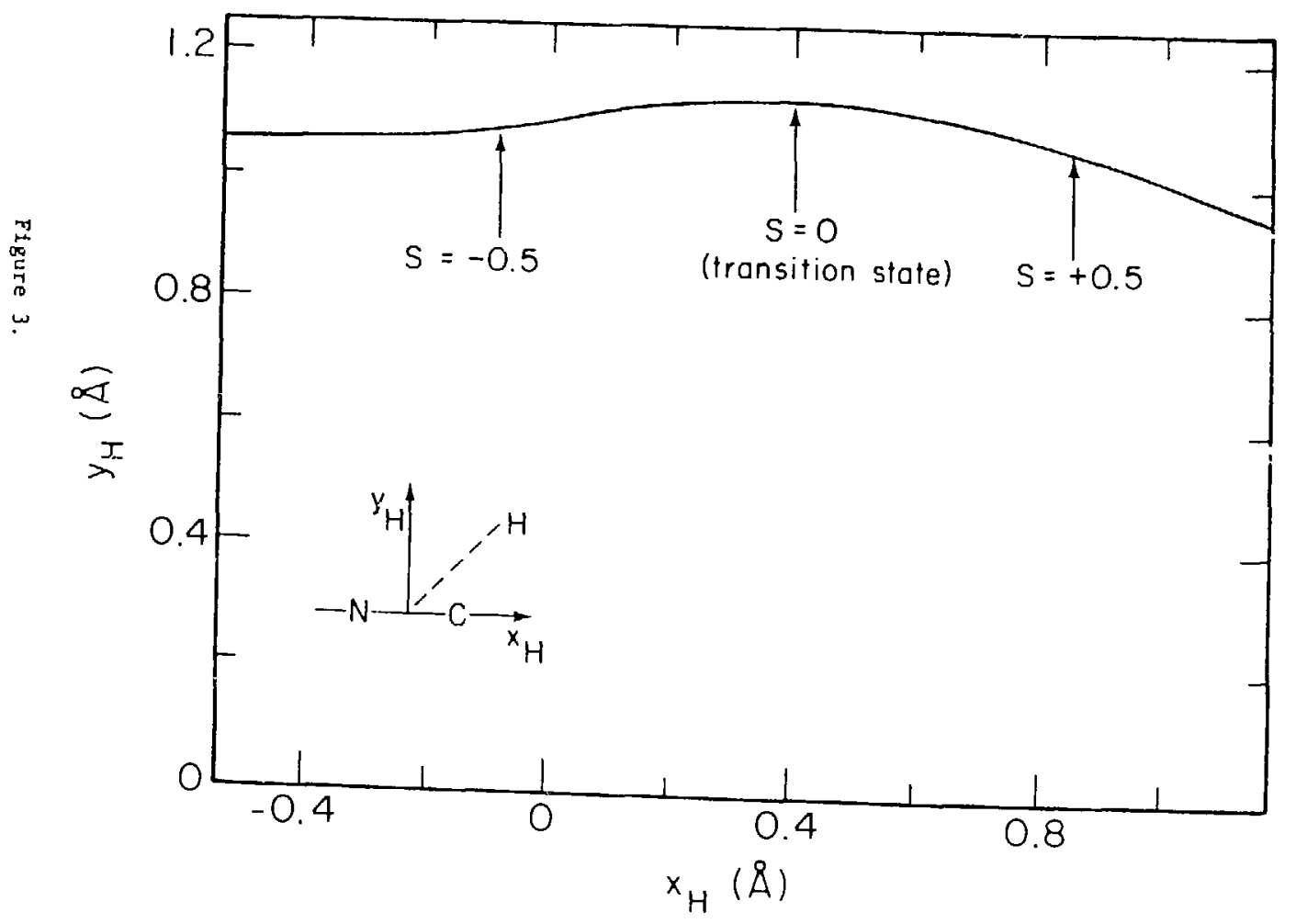

$\varpi$

XBL 806-9863 


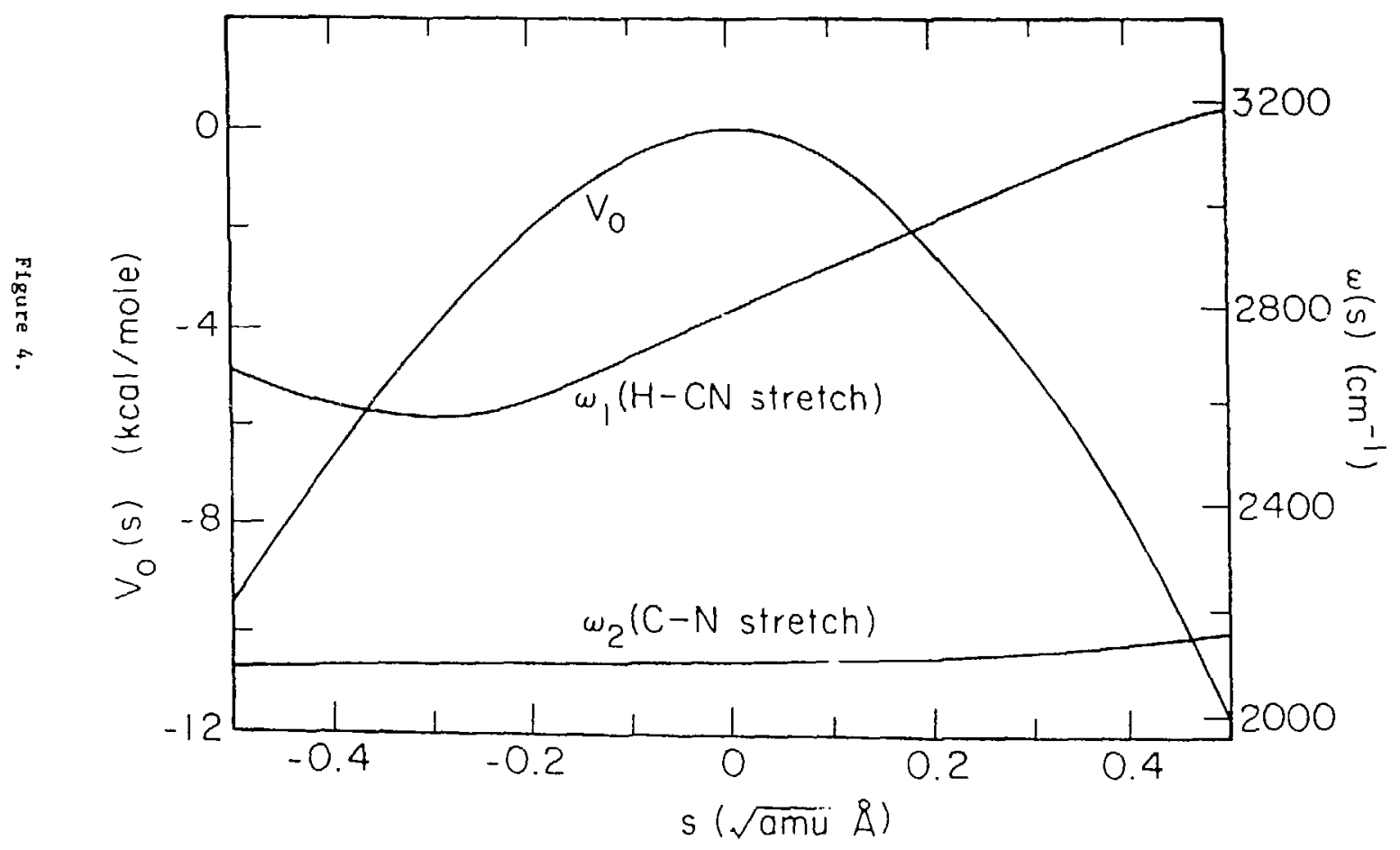




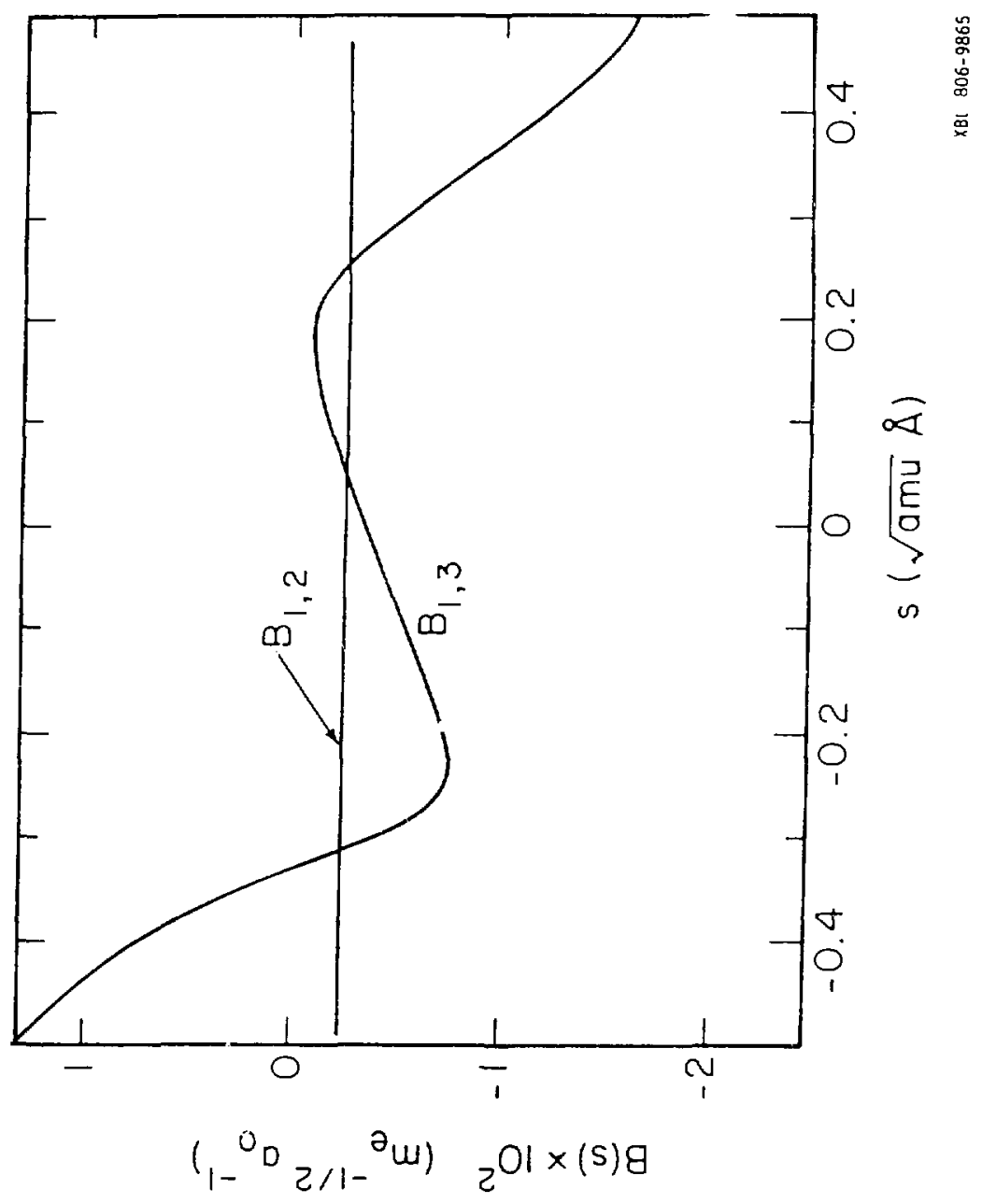

Figure 5. 


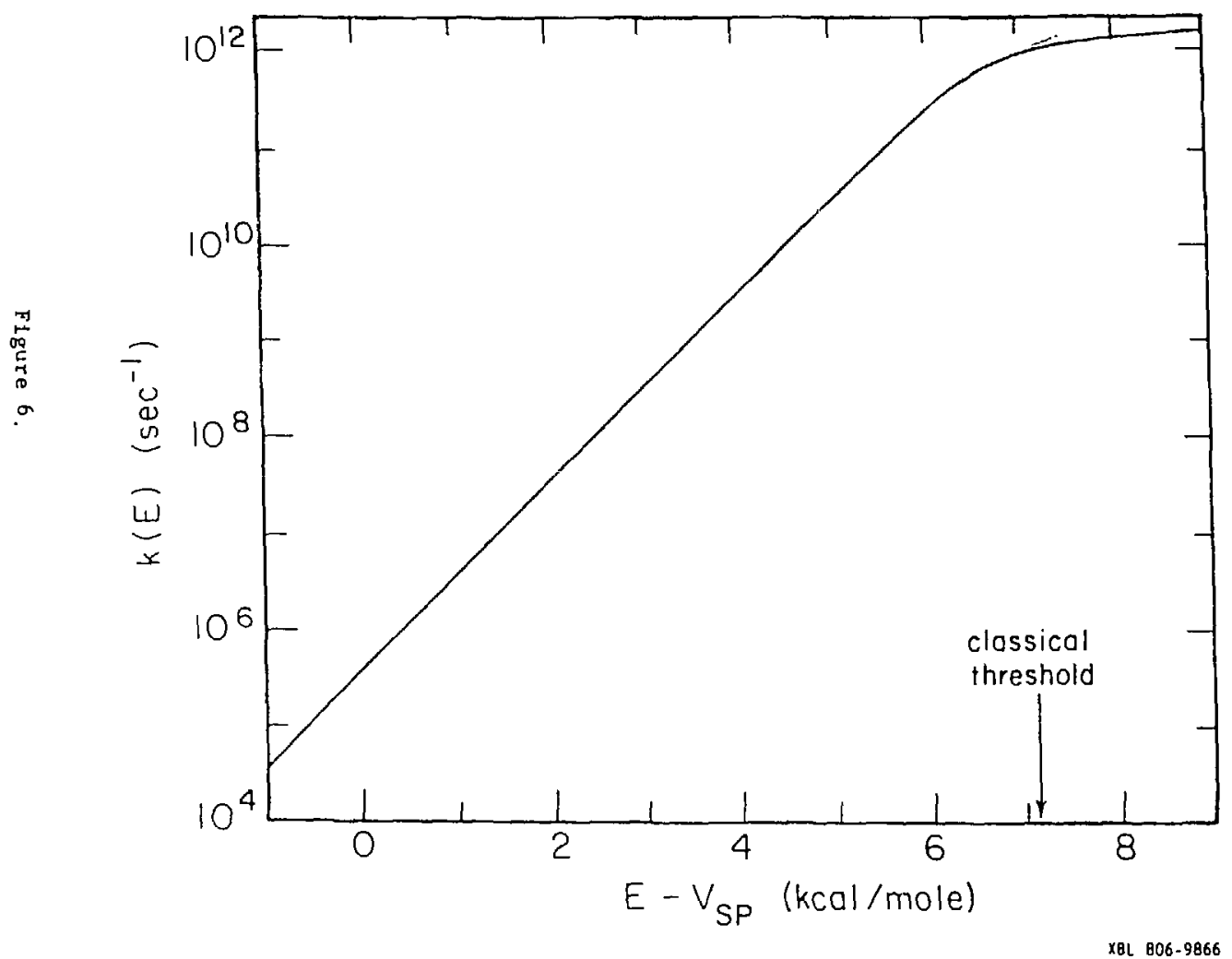




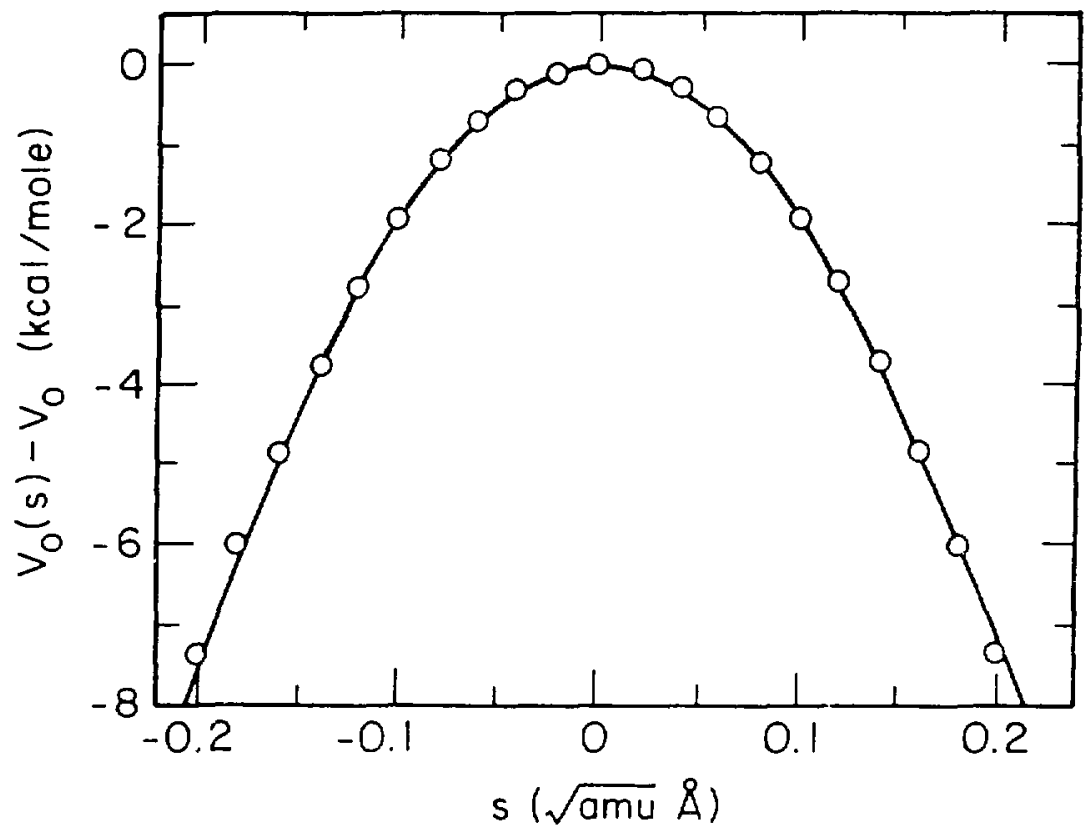

$x 8\llcorner 808-11071$

EIgure 7. 


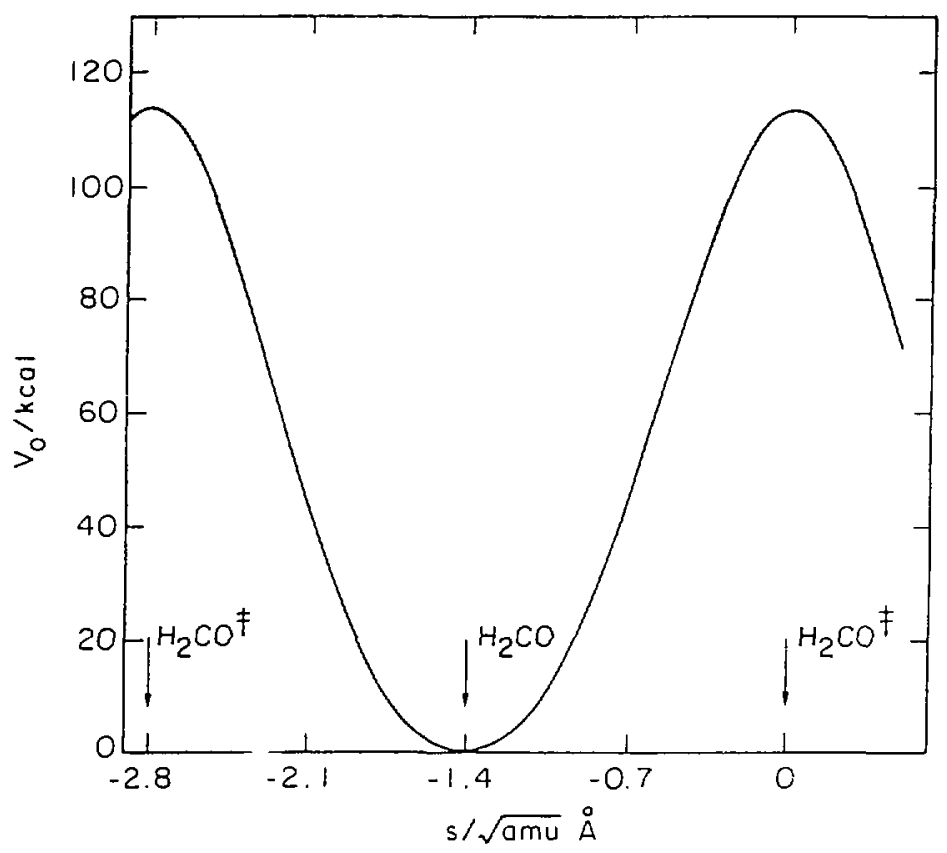

Figure 8 


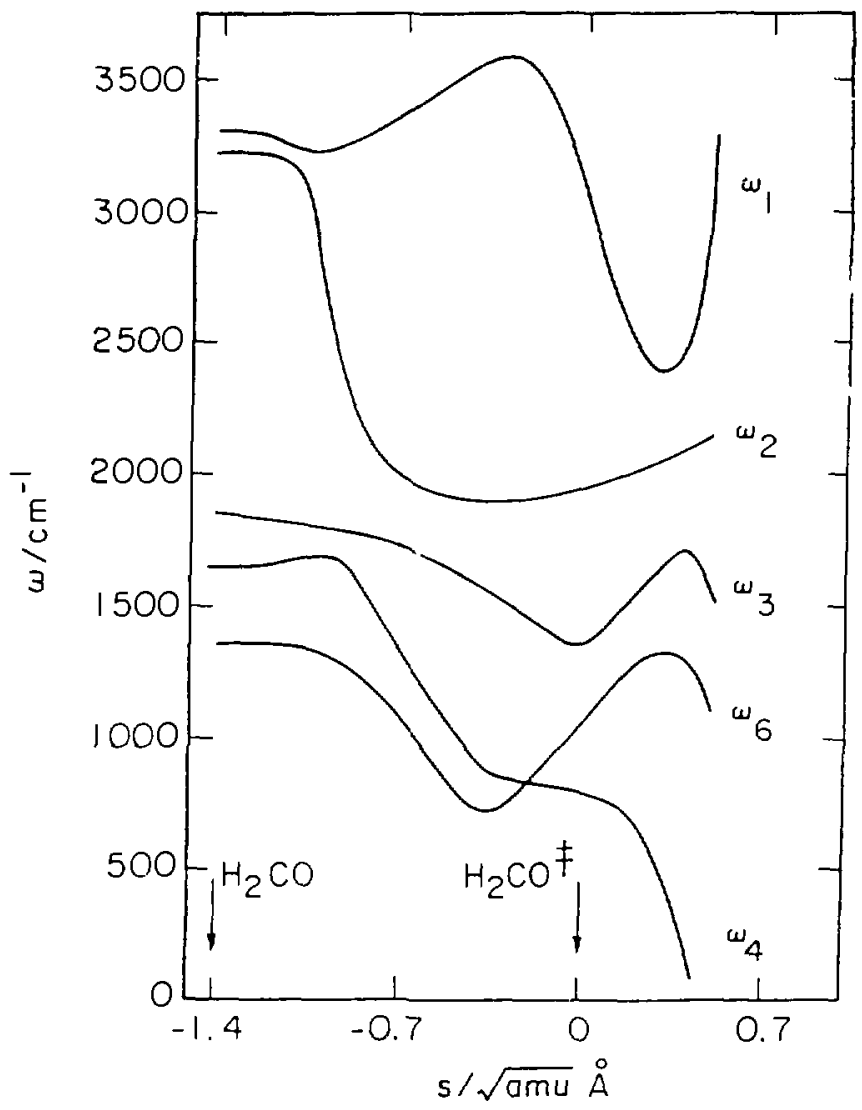

Figure 9 


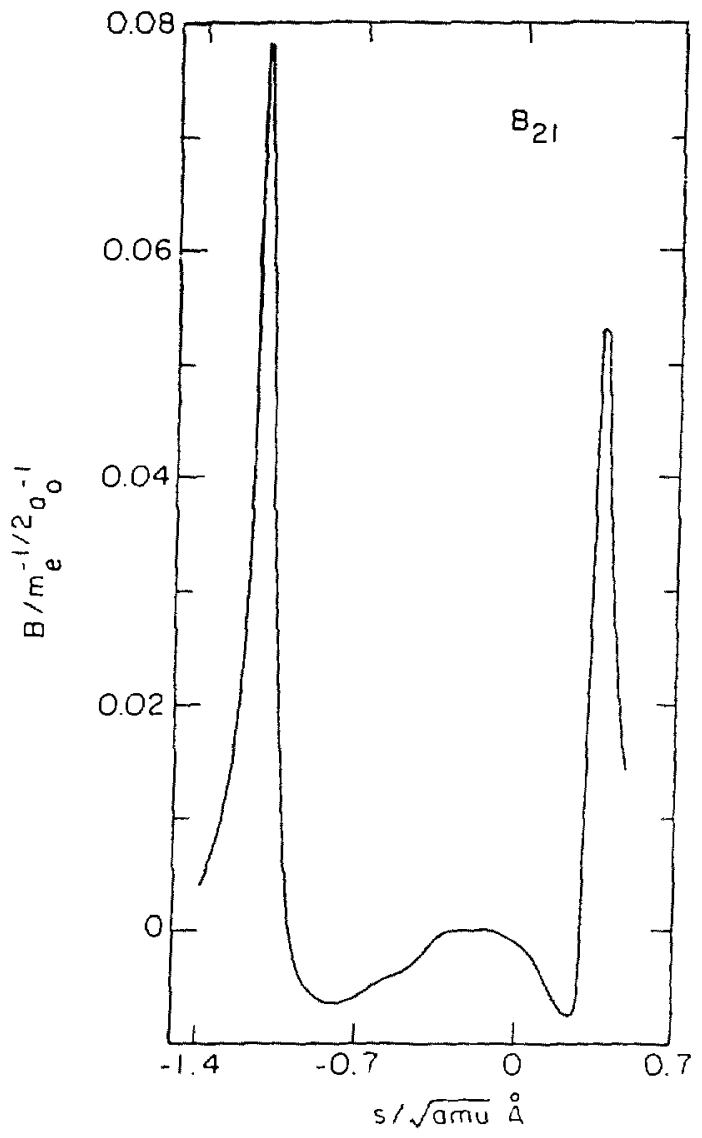

Figure 10a 


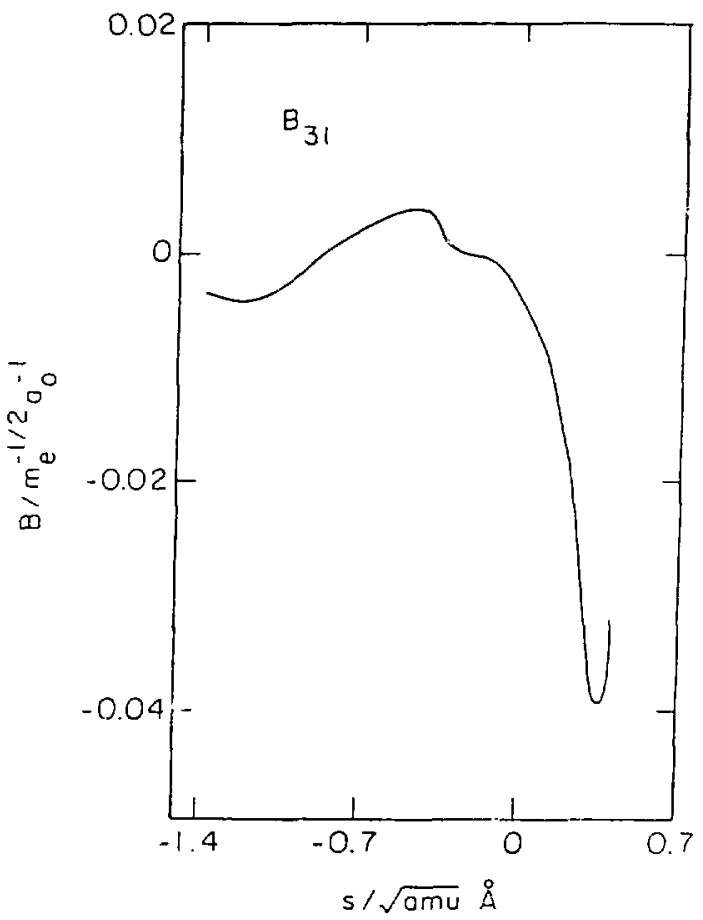

Figure $10 \mathrm{~b}$ 


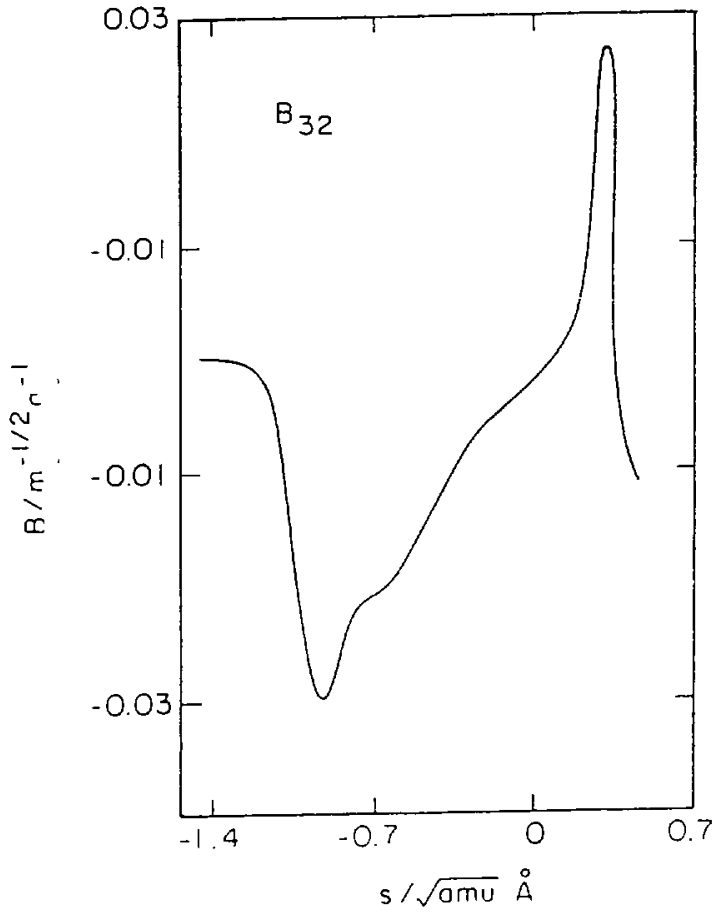

Figure 10c 


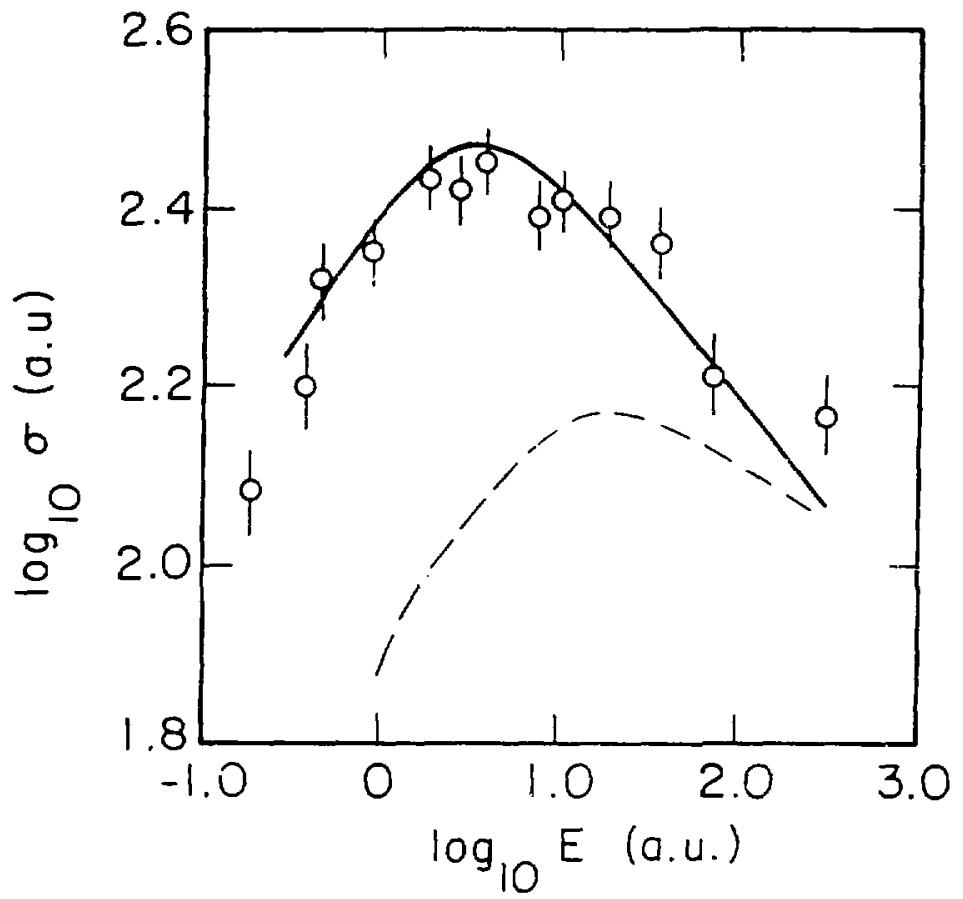

Figure 11 


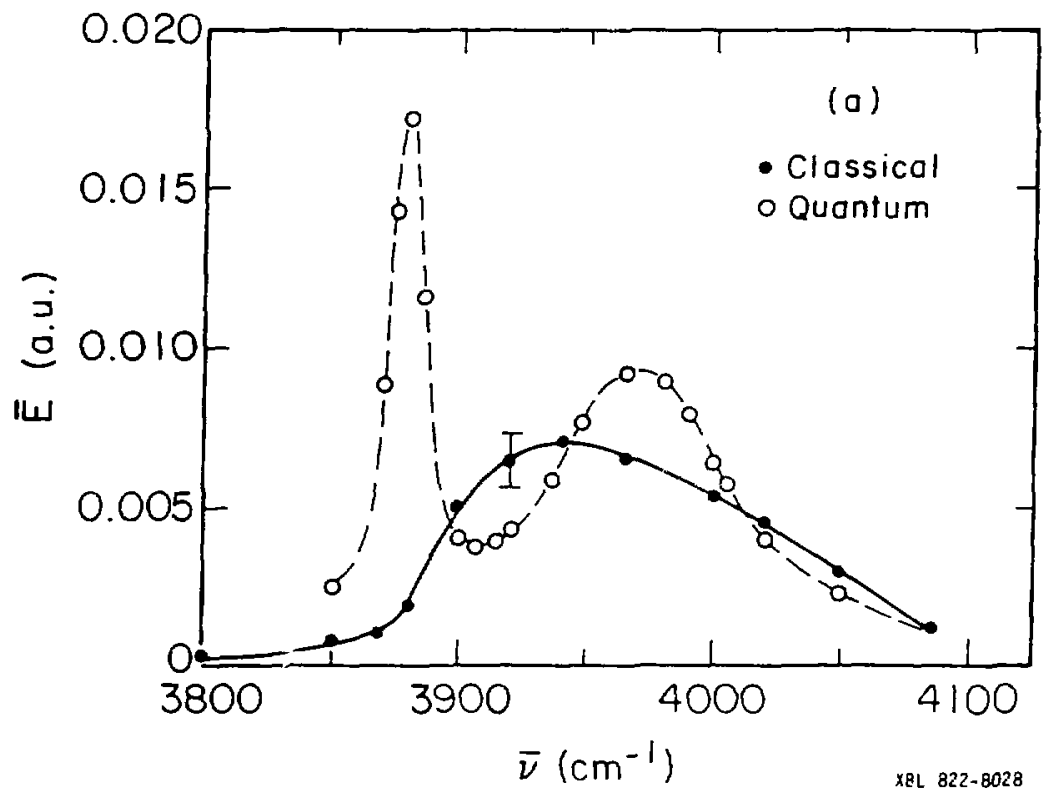

Figure 12a. 


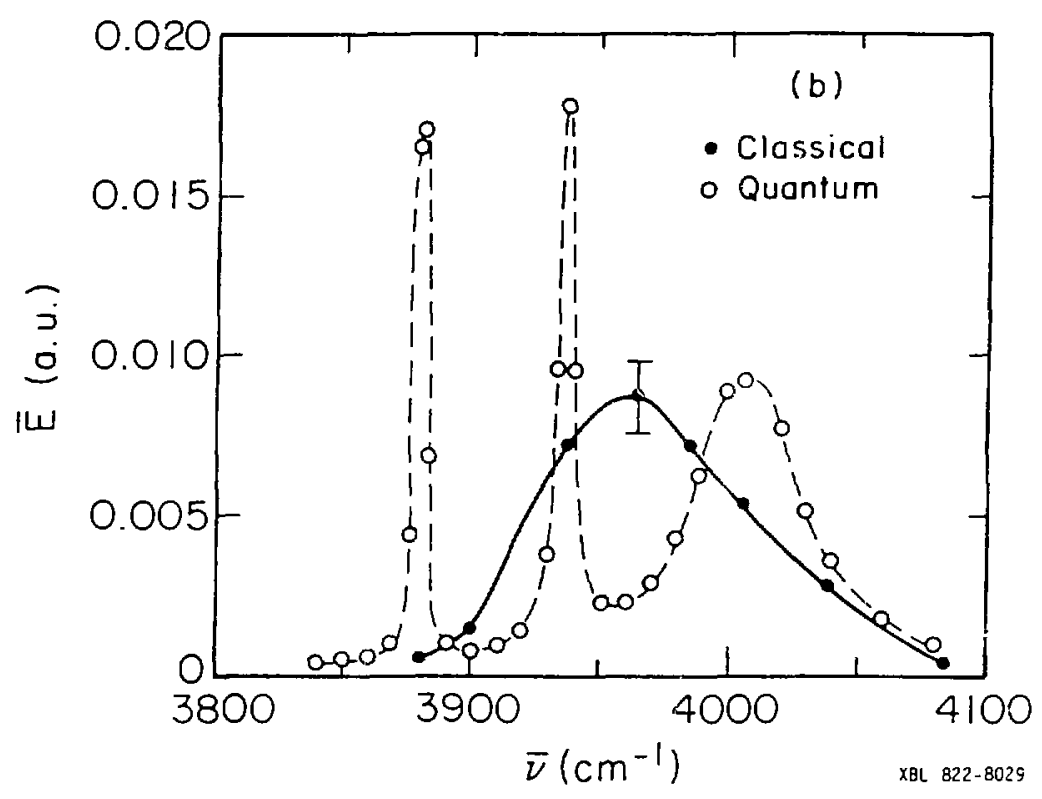

Figure 12b. 


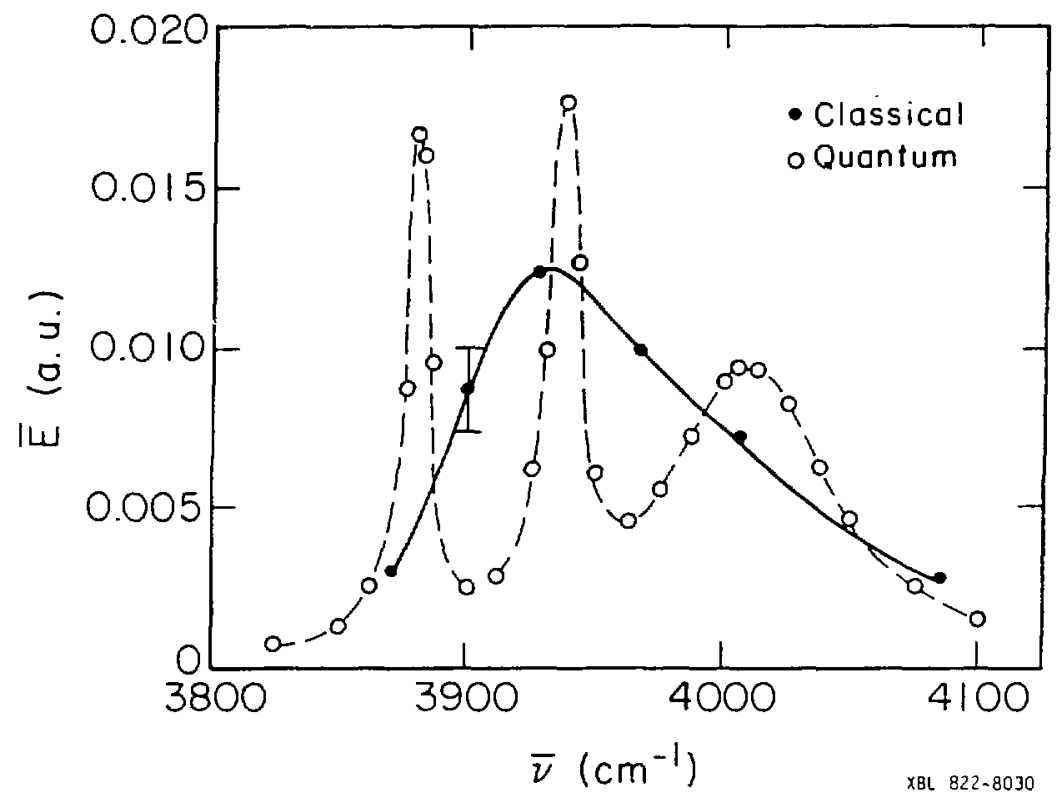

Figure 13. 
145

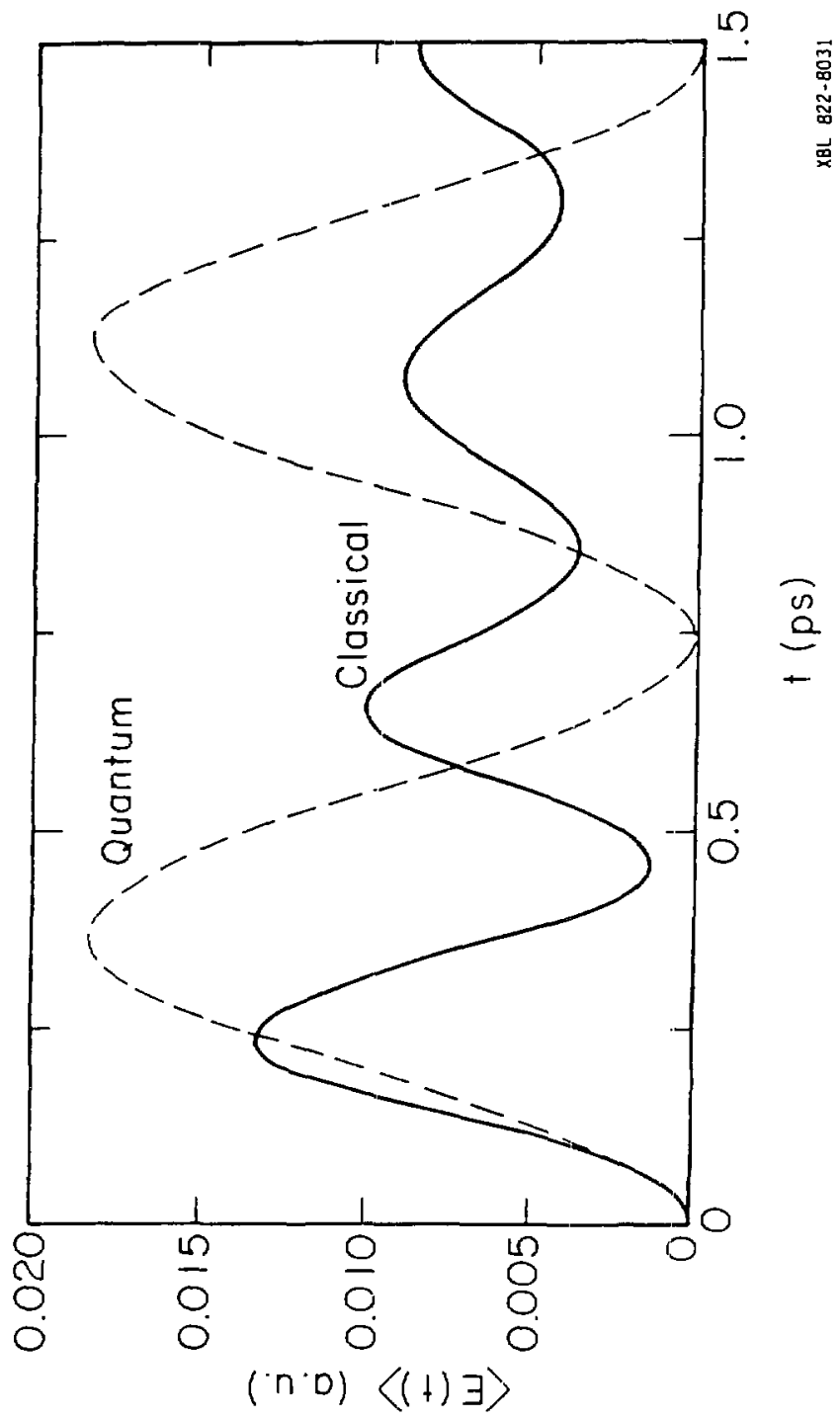

Figure 14. 


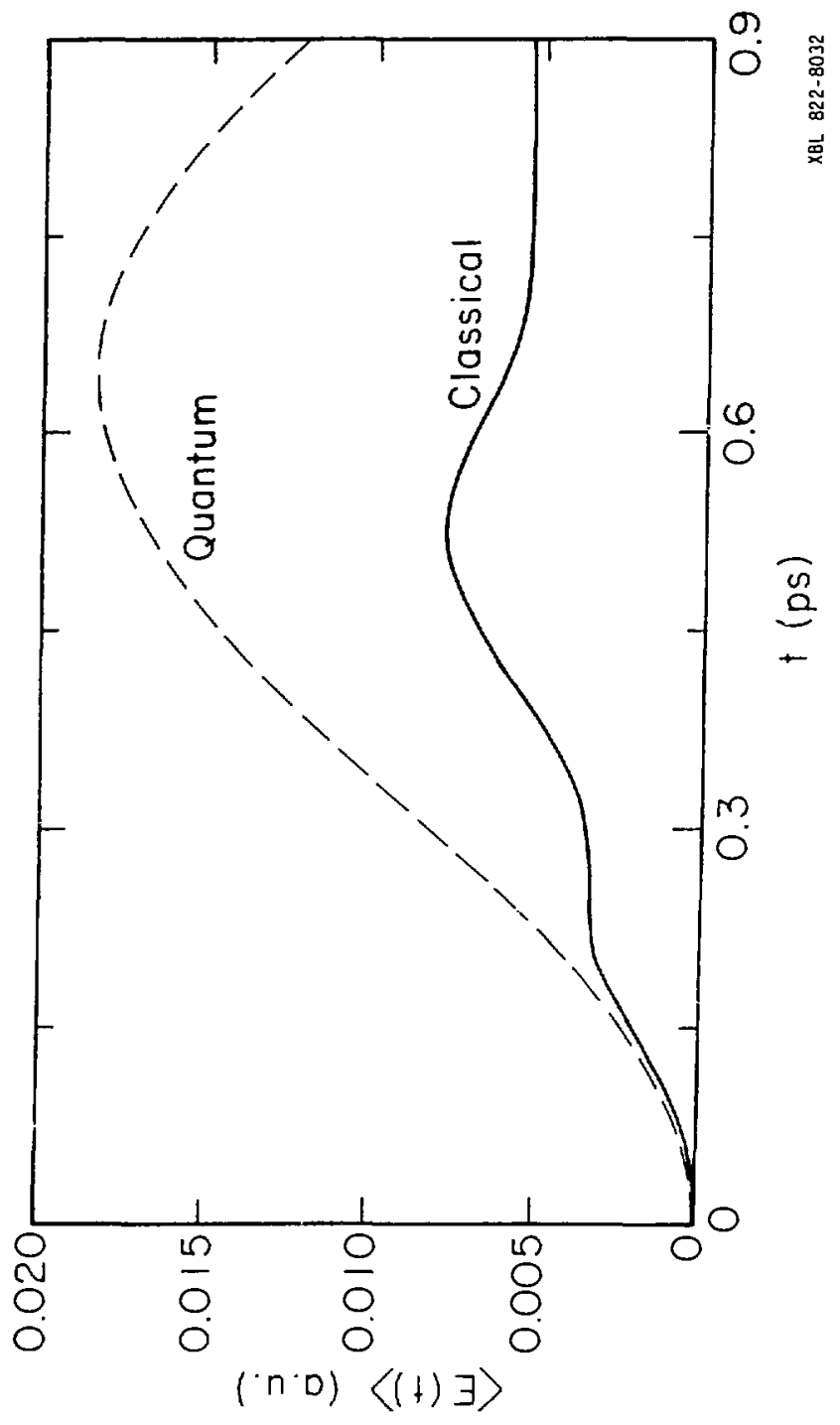

Figure 15. 


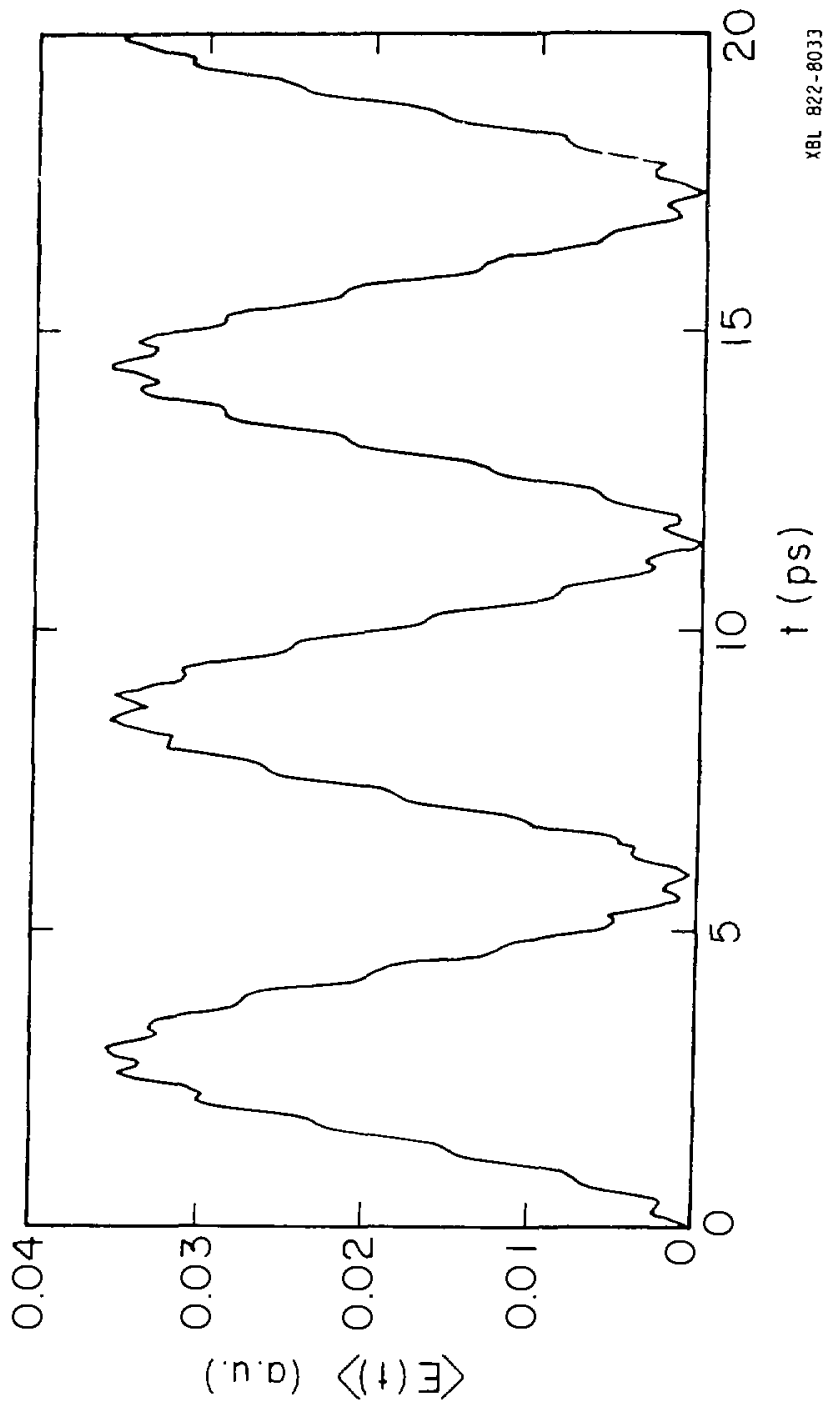

Figure 16. 


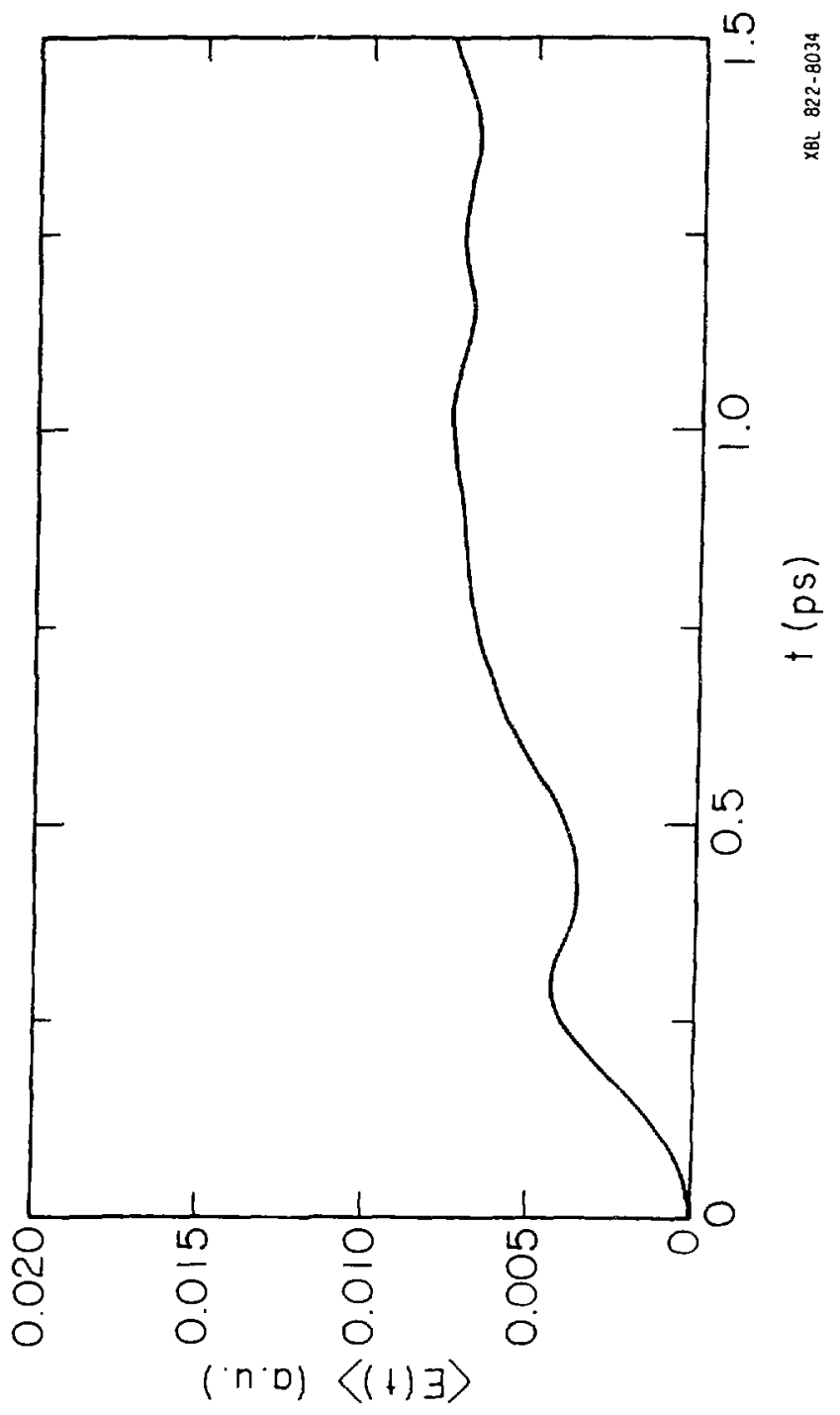

Eigure 17. 

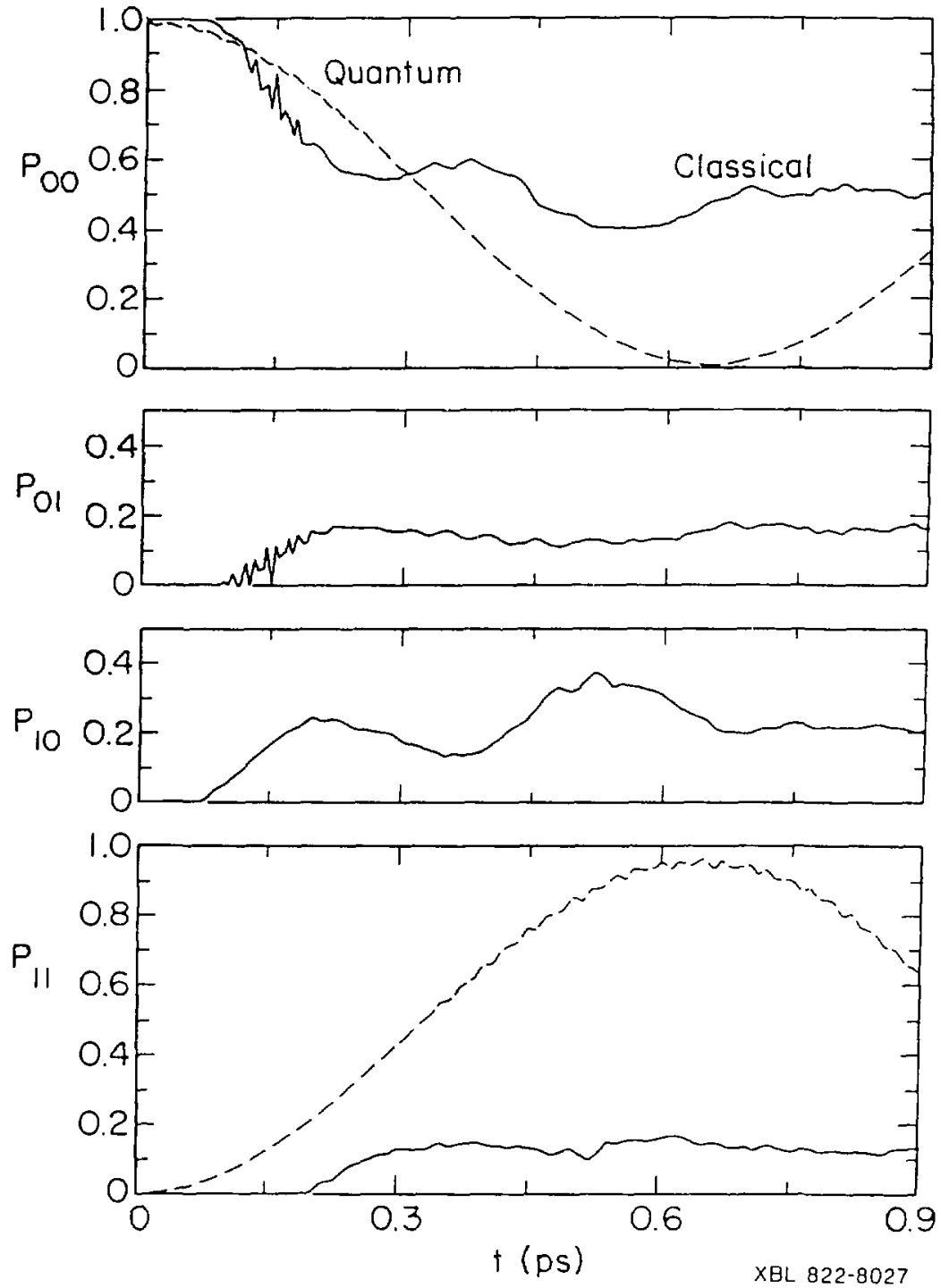

Figure 18. 

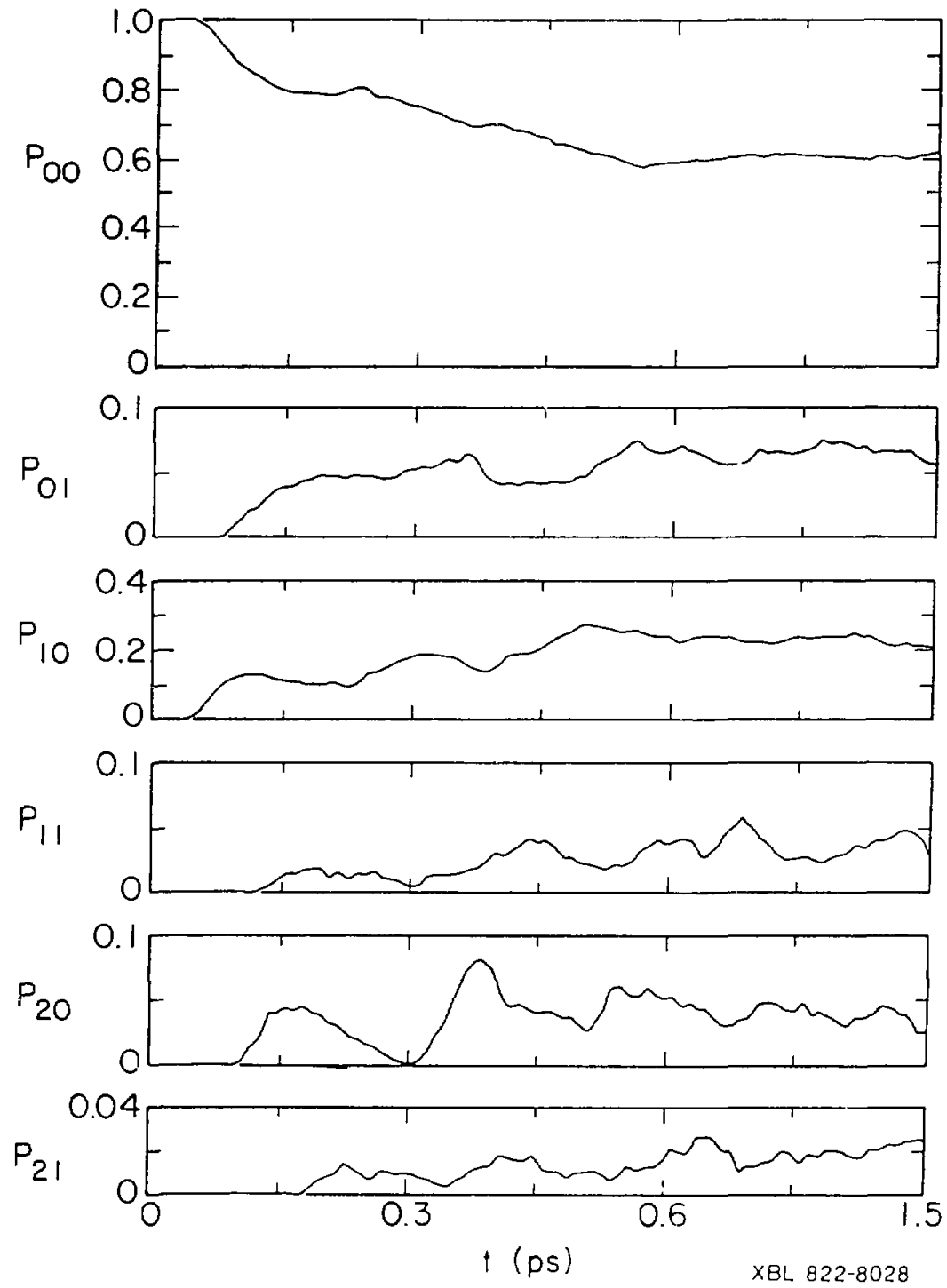

Figure 19. 


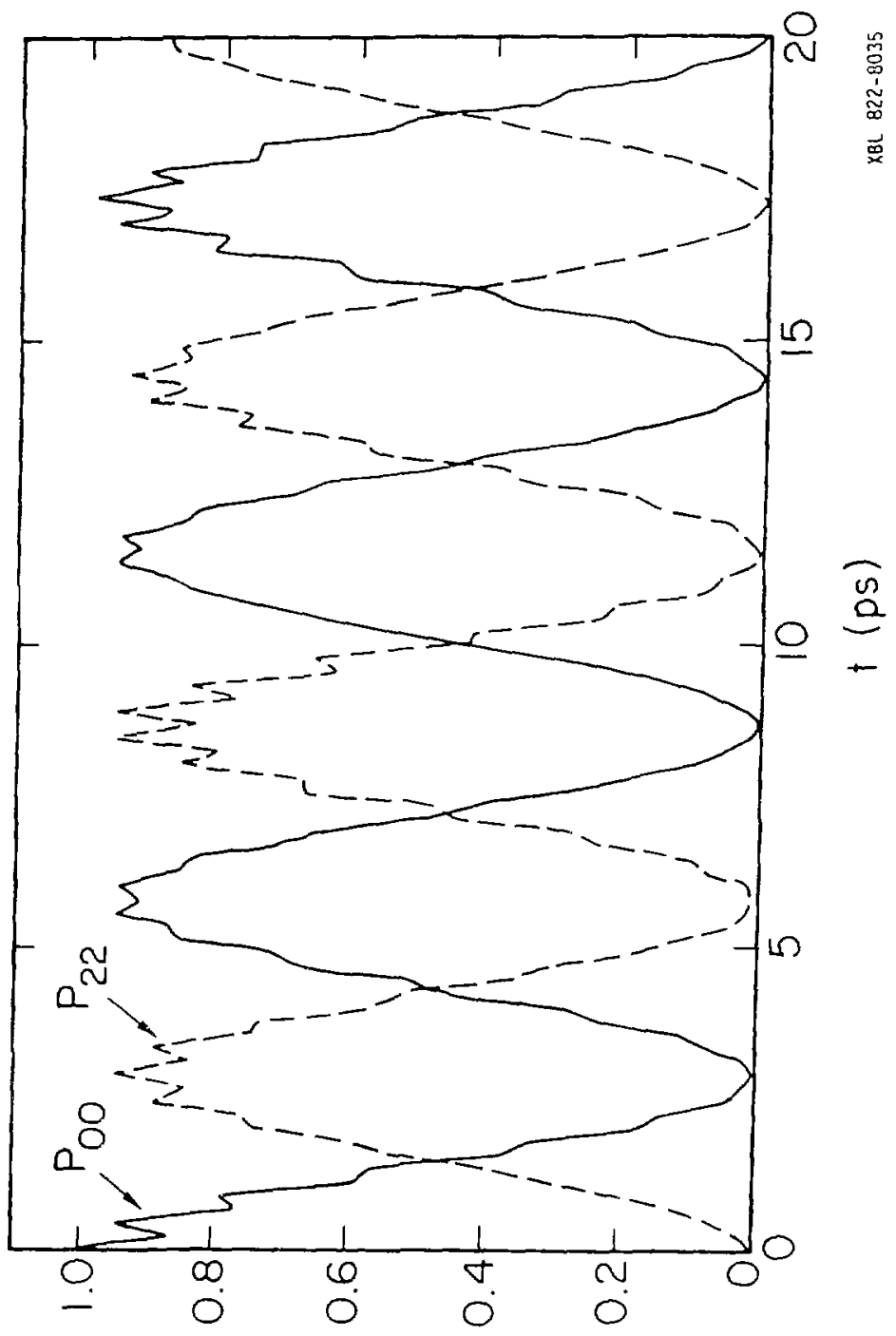

Figure 20. 


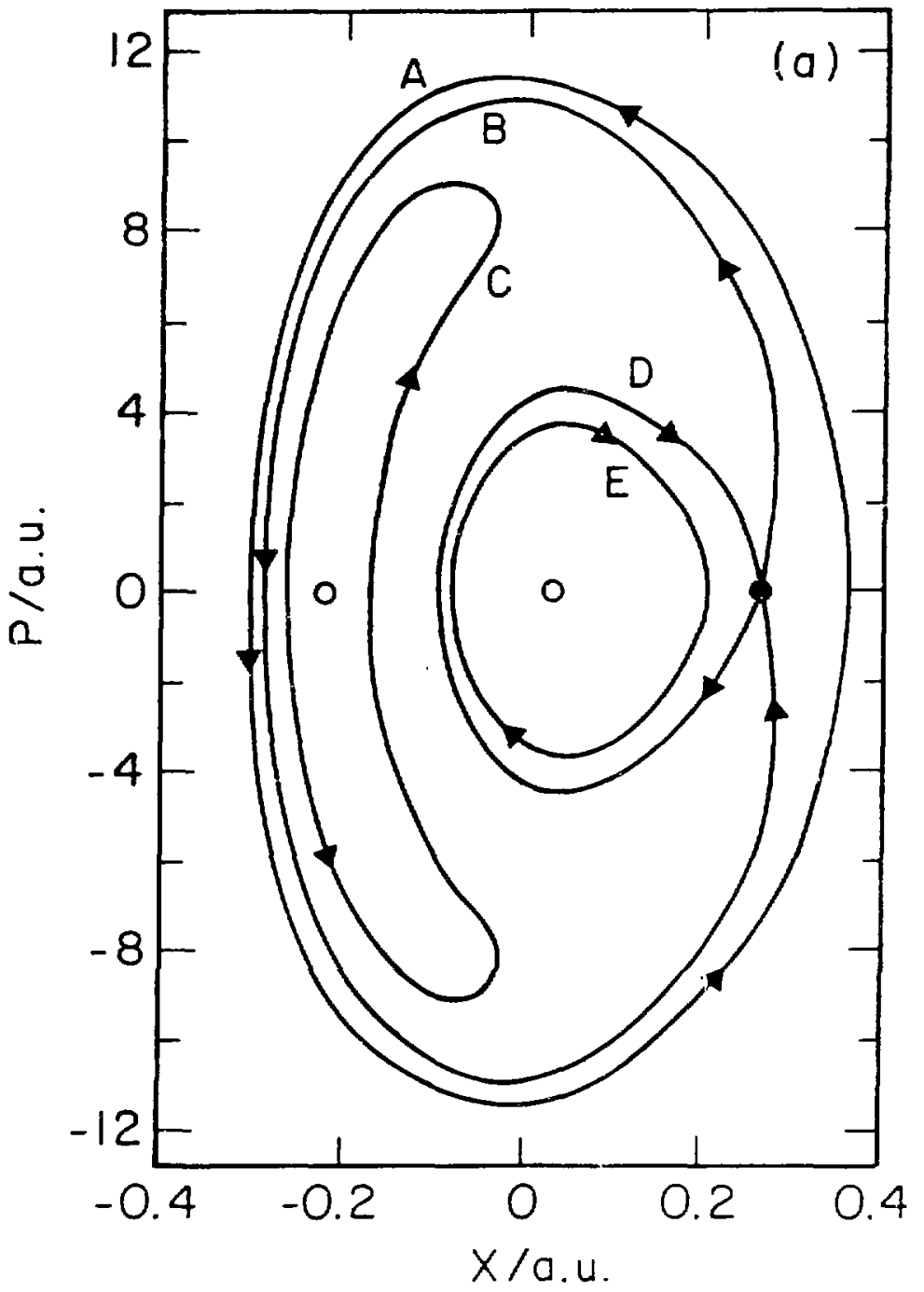

Figure 2 la. 


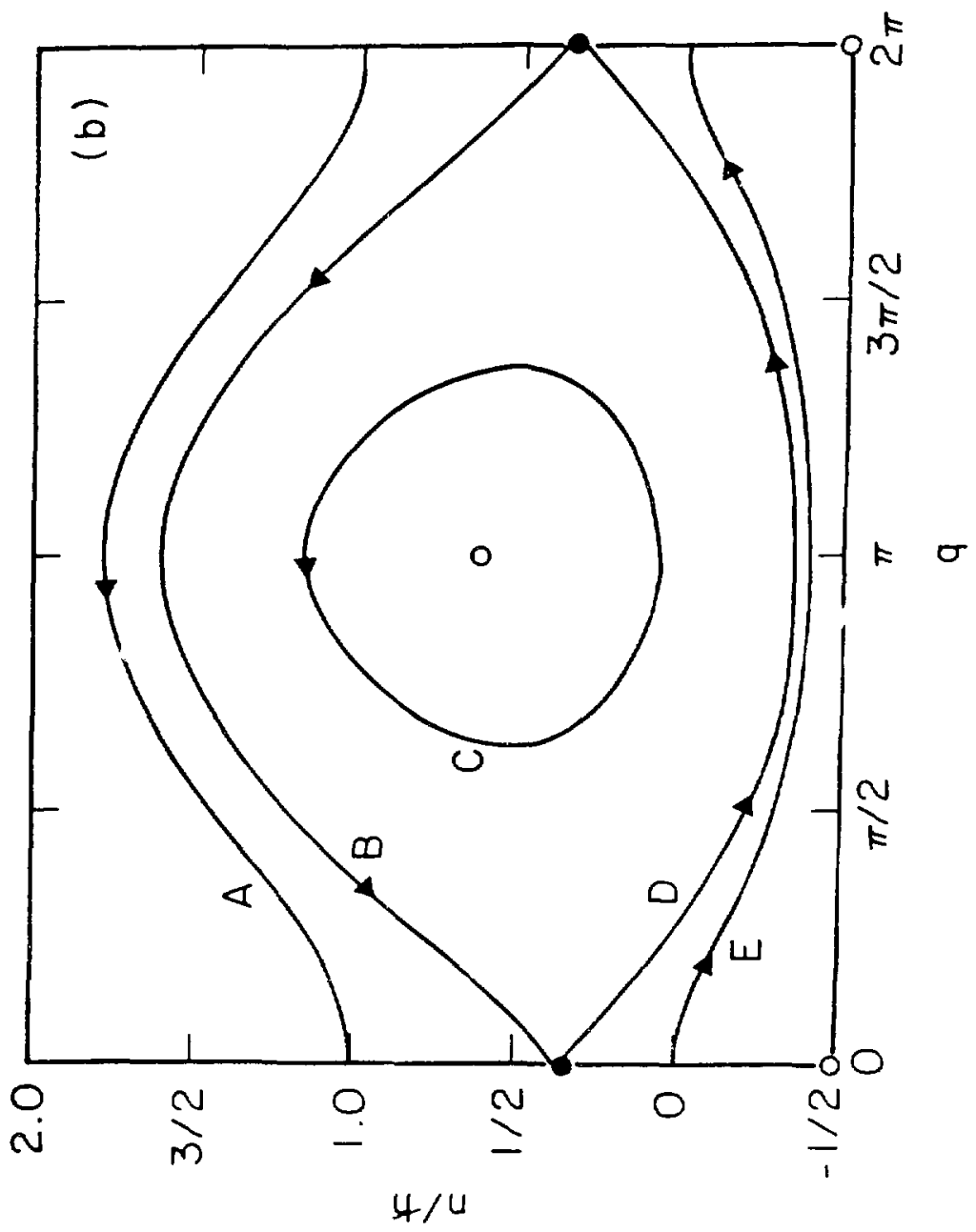

Figure $21 \mathrm{~b}$. 


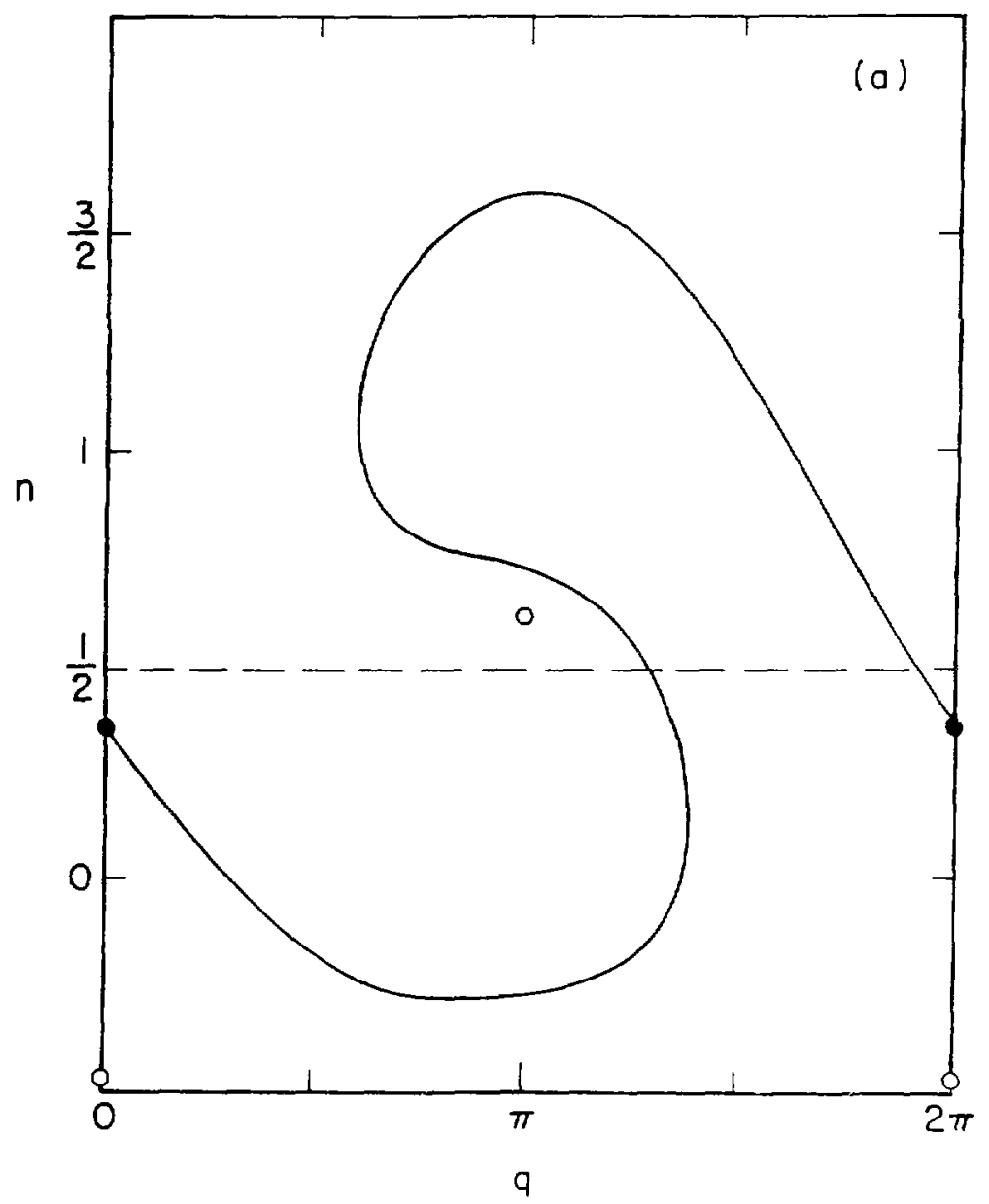

Figure 22a. 


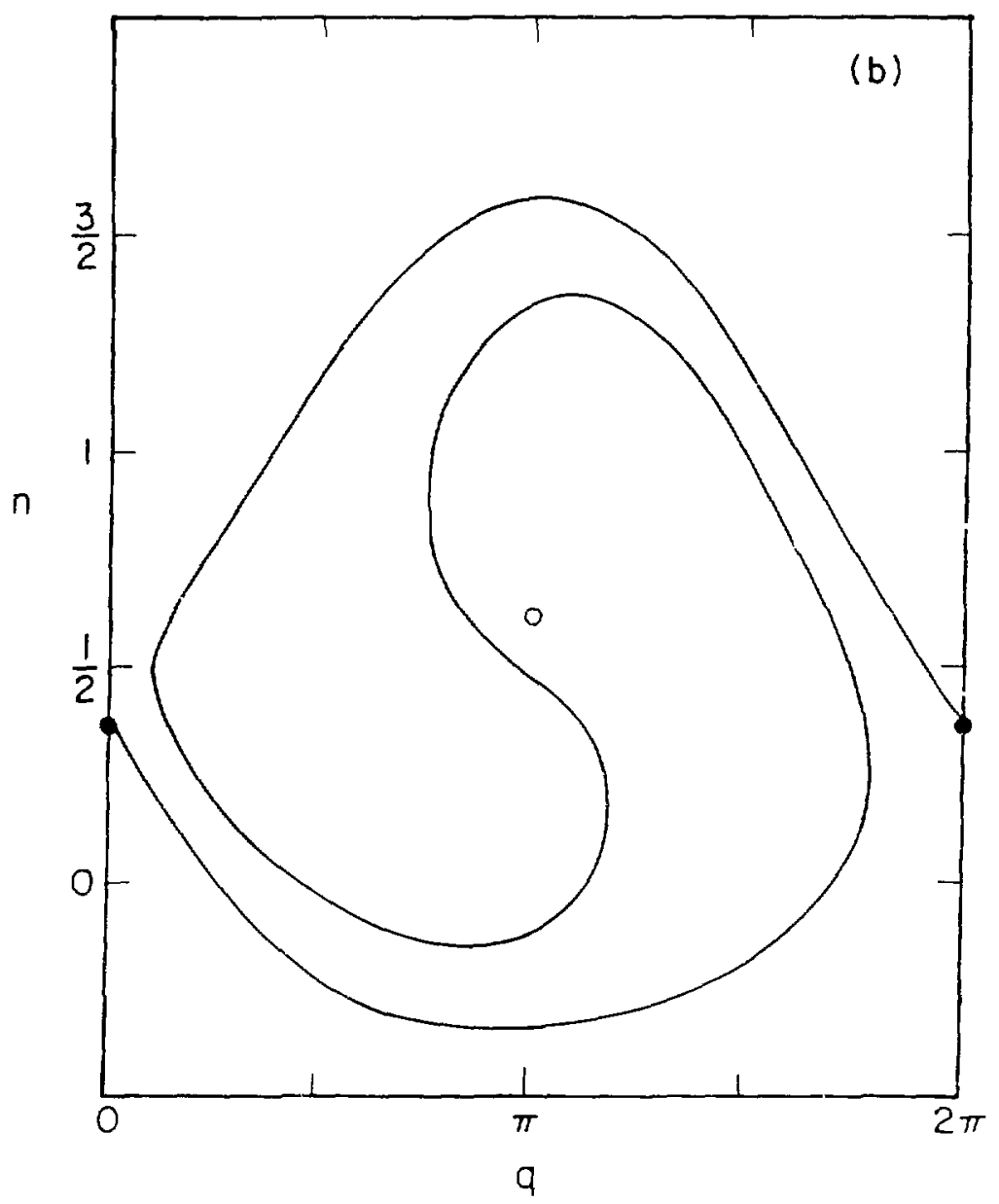

Eigure $22 \mathrm{~b}$. 


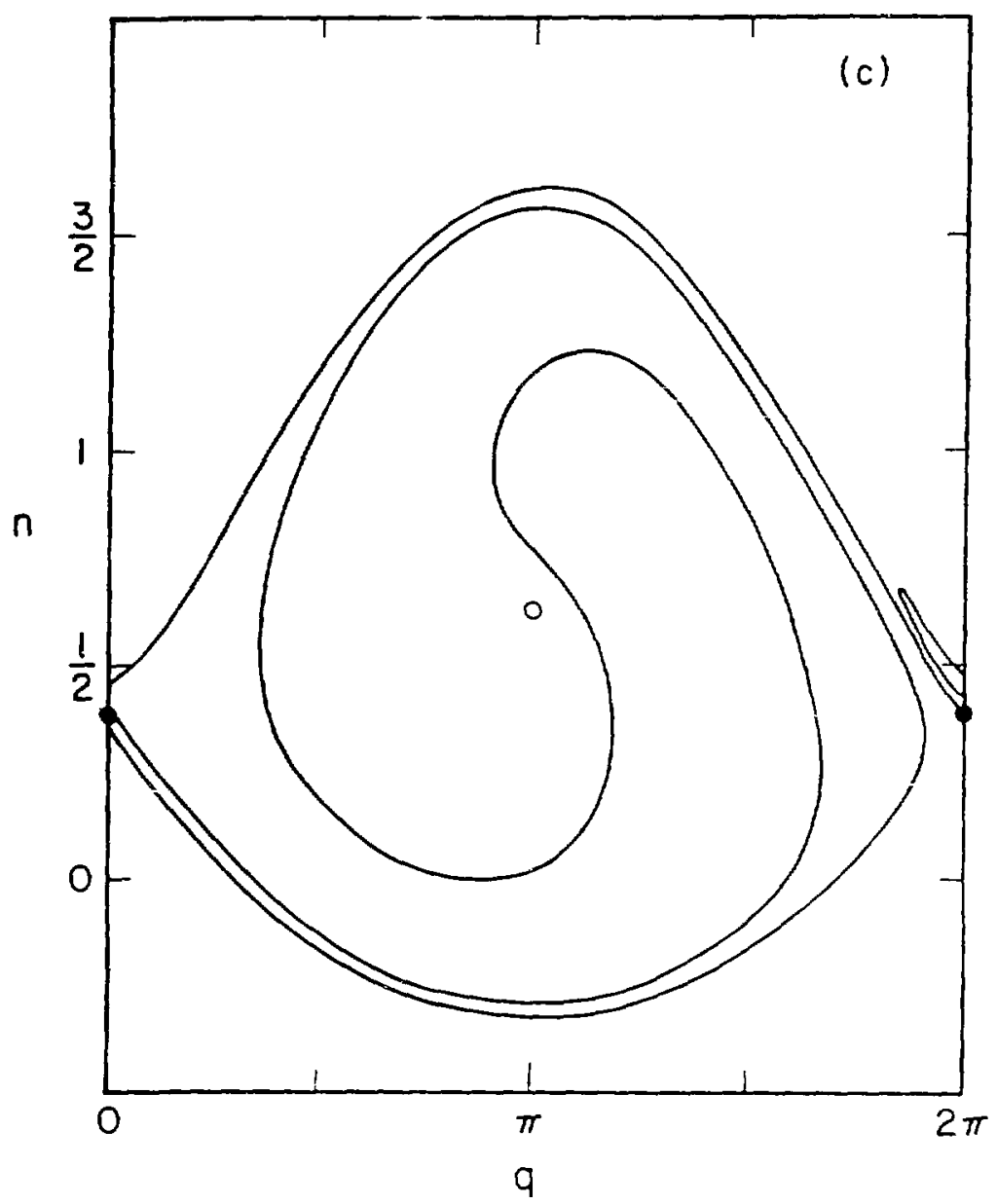

F1gure 22c. 


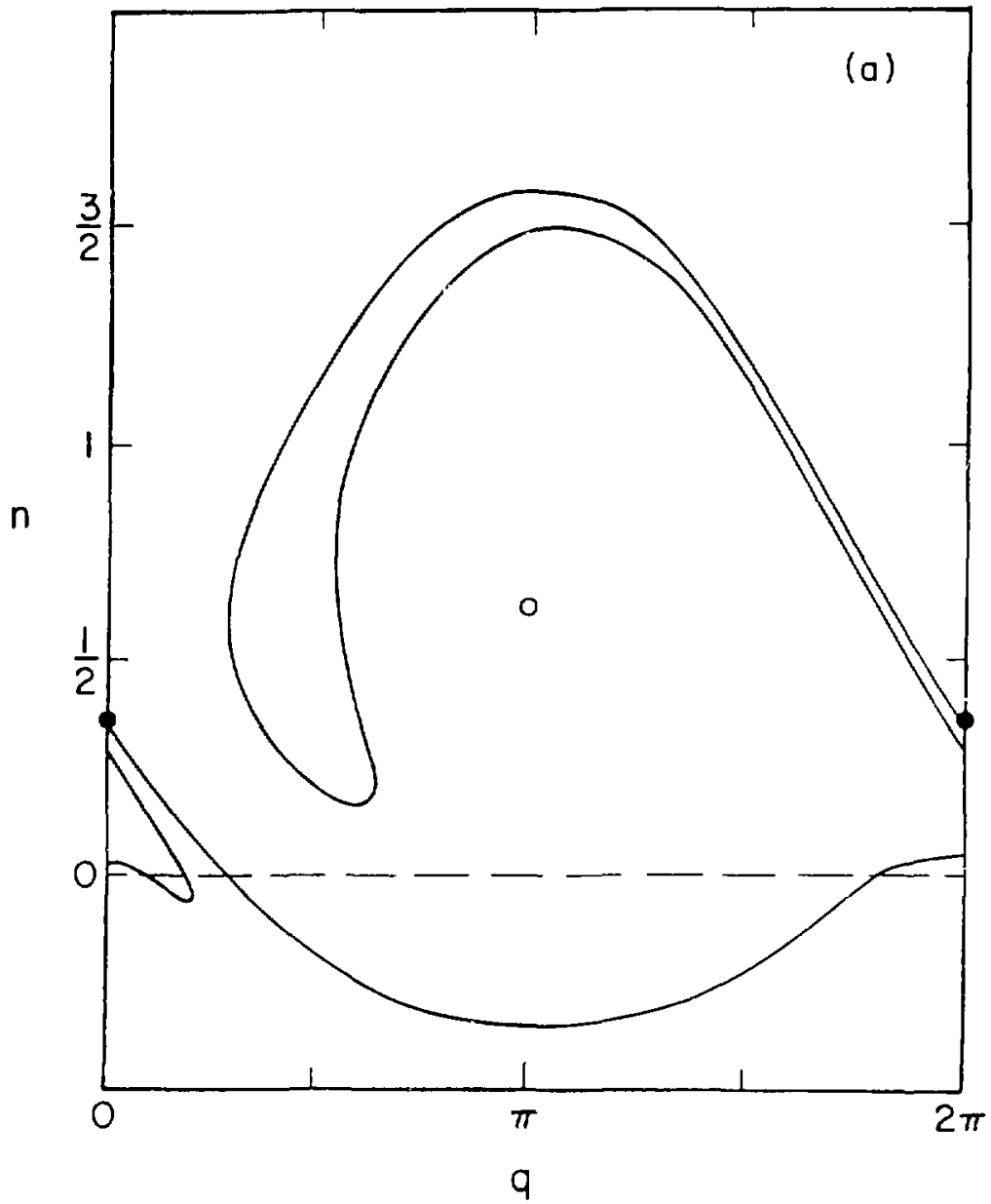

Figure $23 a$. 


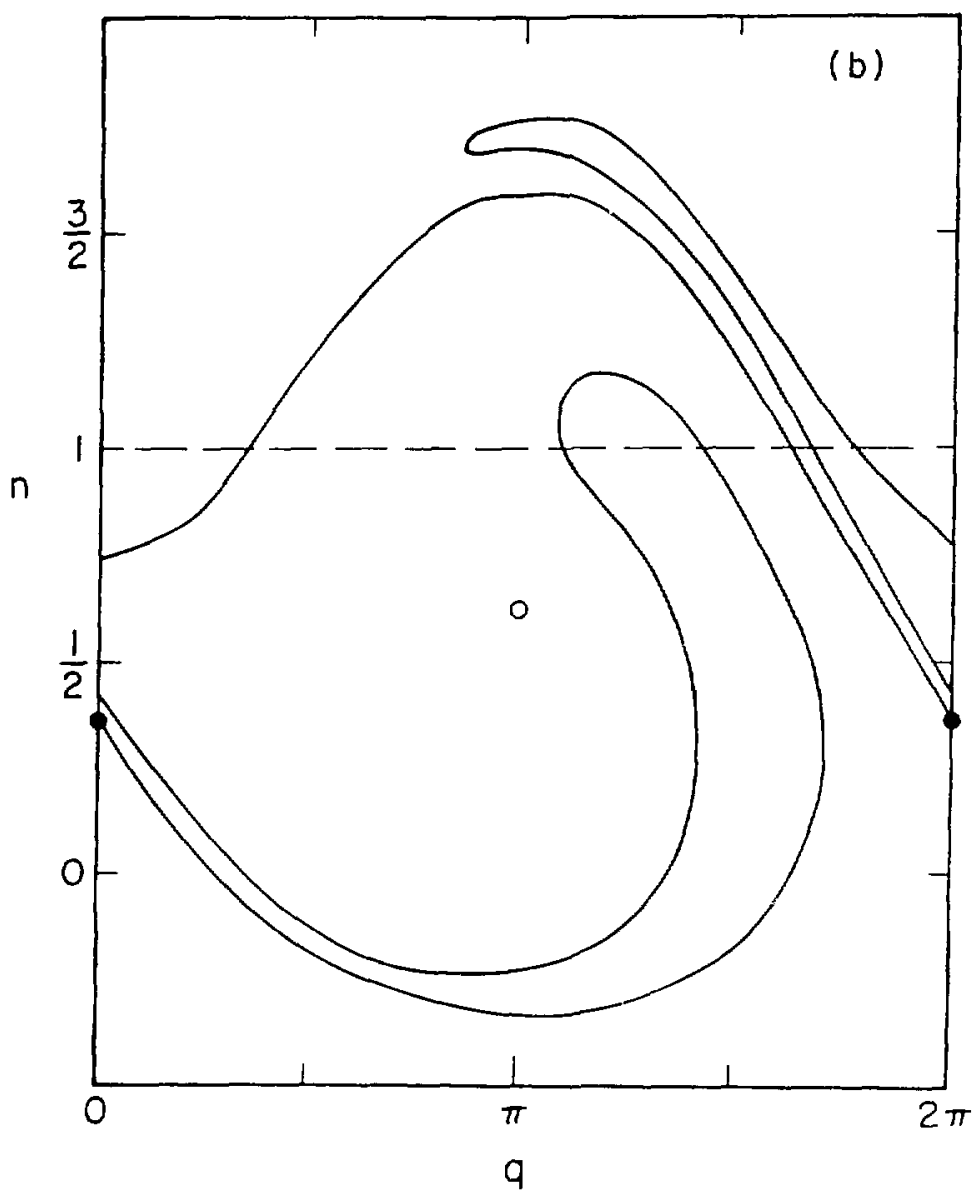

Figure $23 b$. 


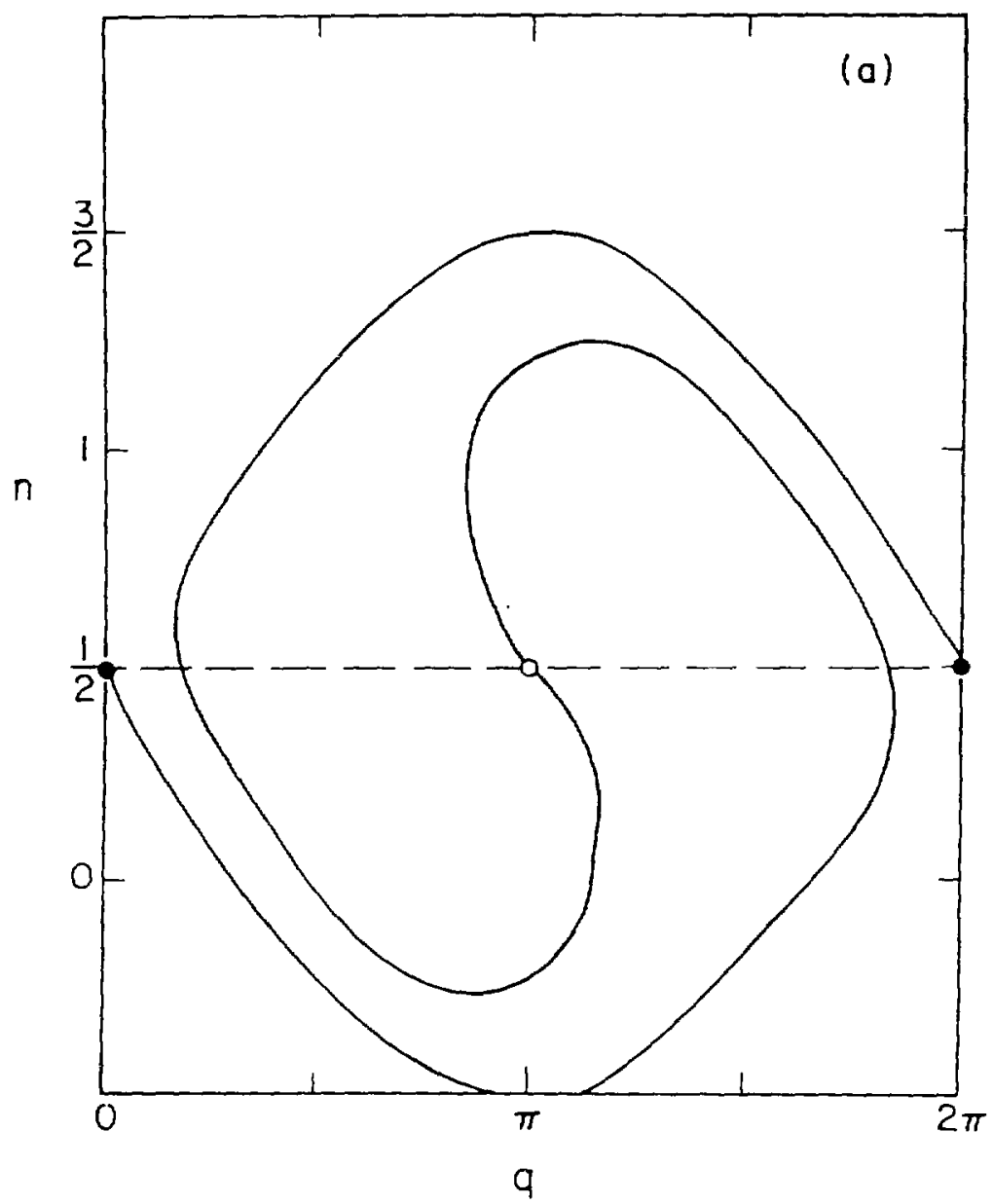

Figure 24a. 


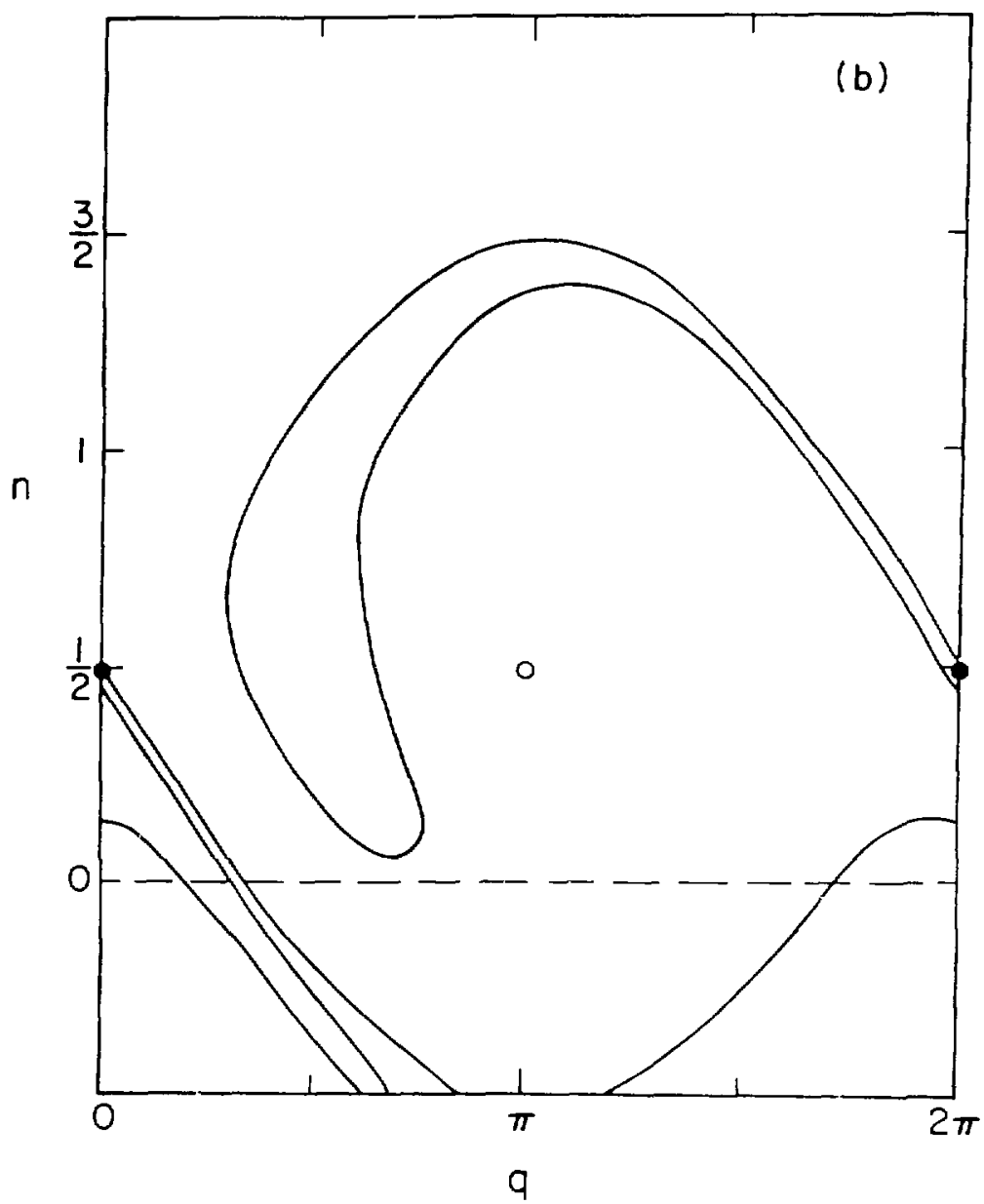

Figure 24b. 


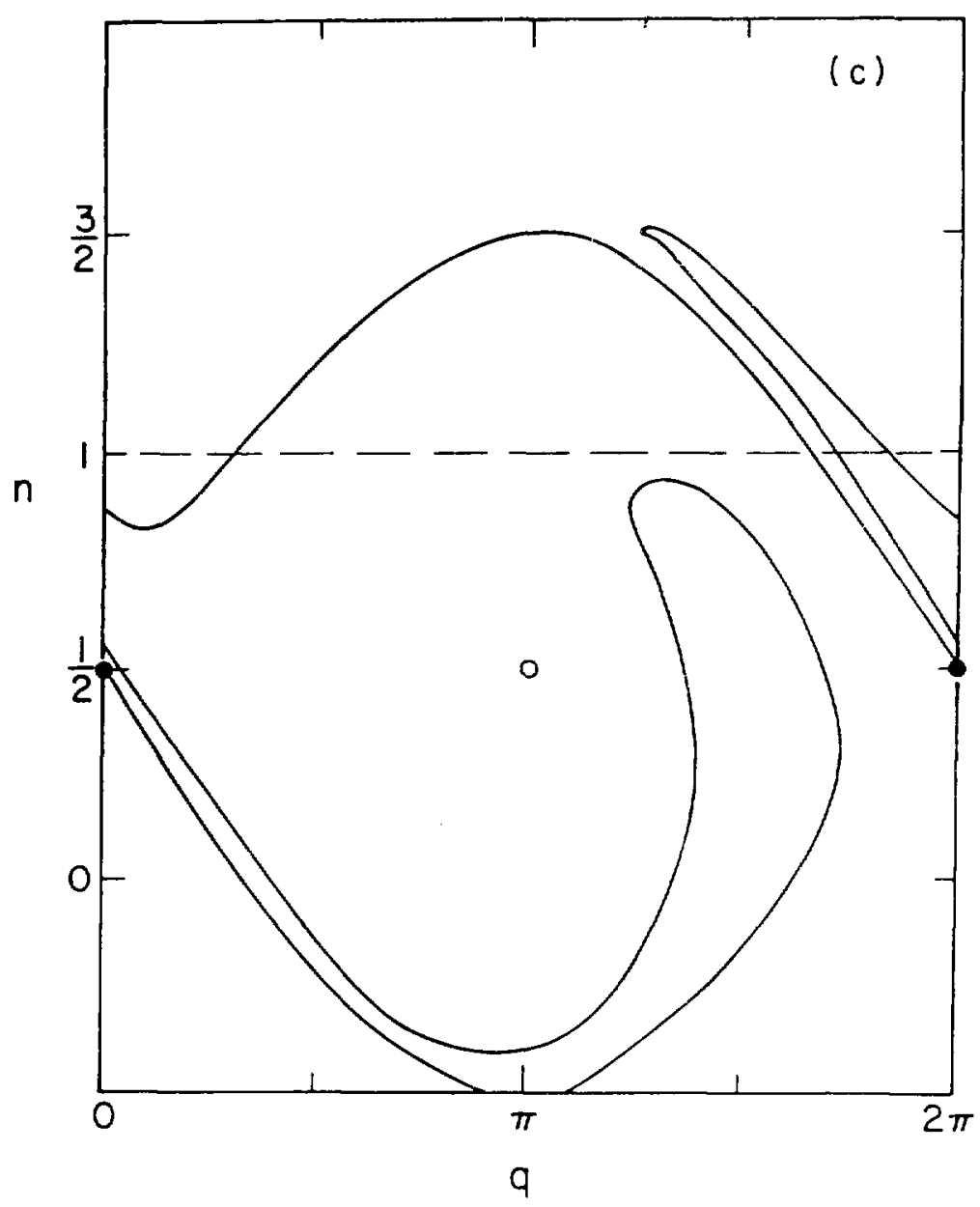

Figure 24c. 


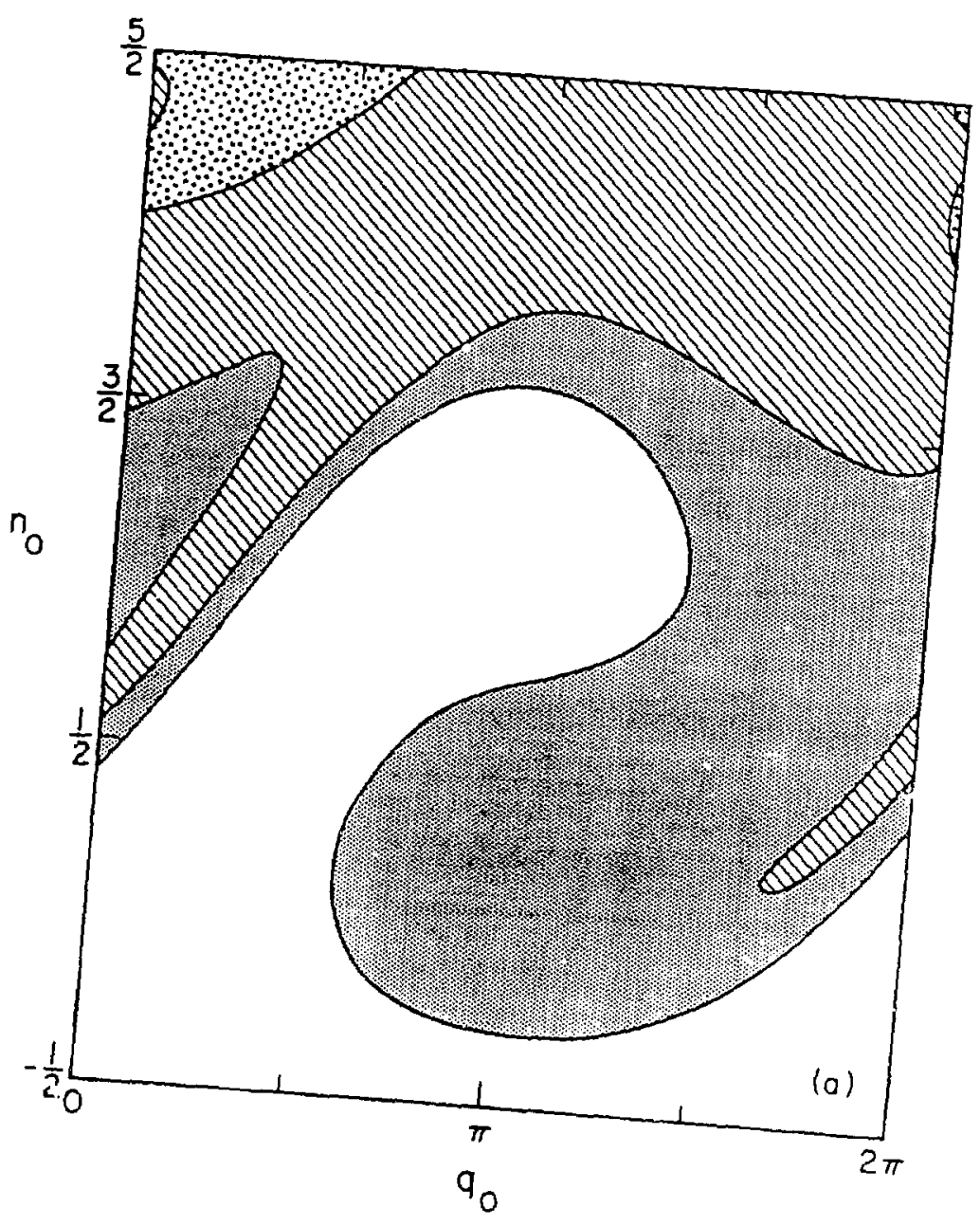

Figure 25a. 


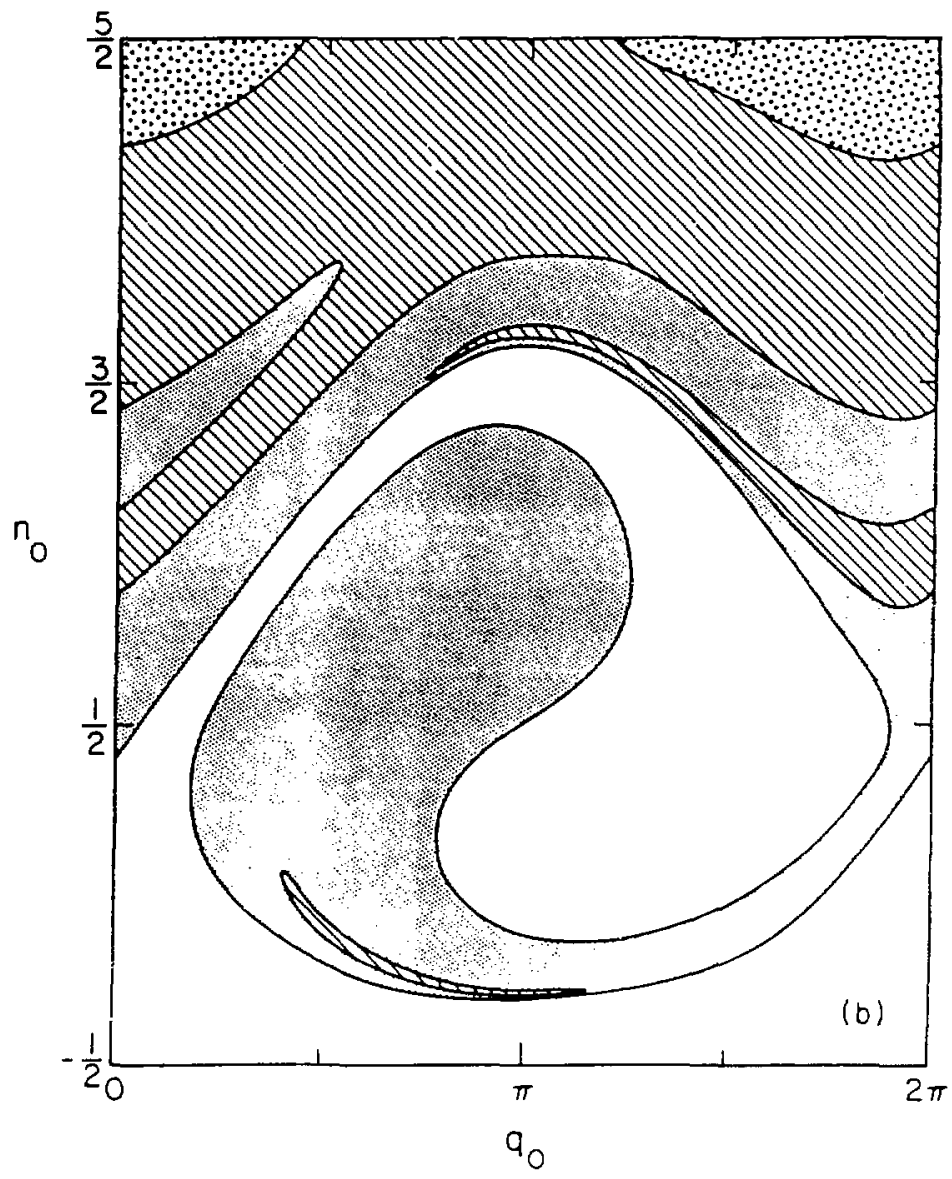

Figure 25b. 


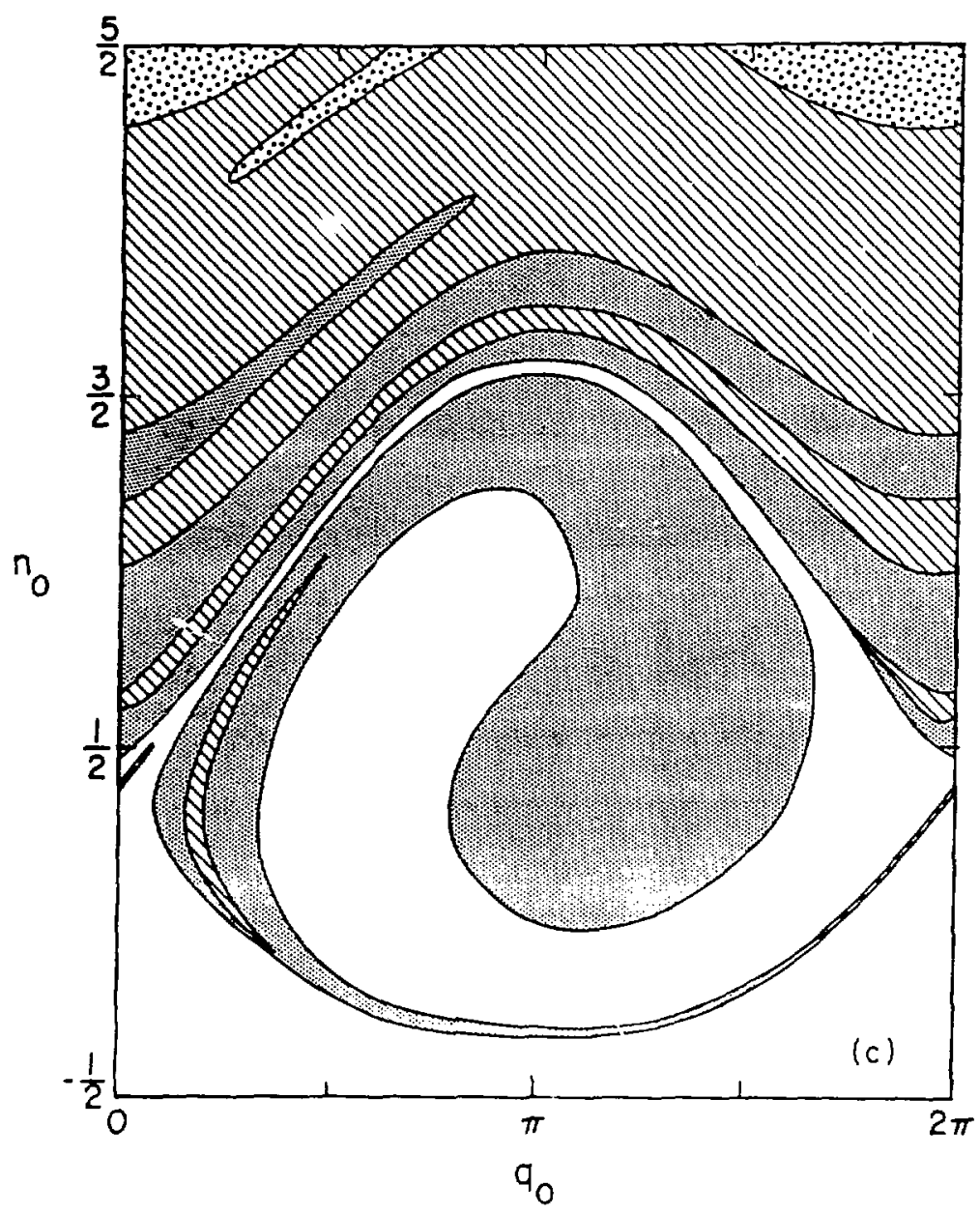

Figure $25 c$. 